\title{
A stable partitioned FSI algorithm for incompressible flow and deforming beams
}

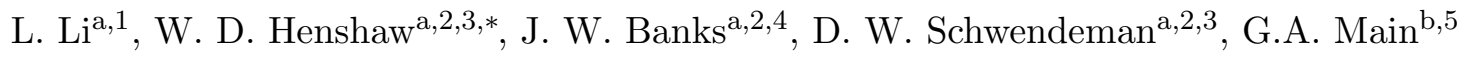 \\ ${ }^{a}$ Department of Mathematical Sciences, Rensselaer Polytechnic Institute, Troy, NY 12180, USA. \\ ${ }^{b}$ Department of Civil and Environmental Engineering, Duke University, Durham, NC, 27708, USA.
}

\begin{abstract}
An added-mass partitioned (AMP) algorithm is described for solving fluid-structure interaction (FSI) problems coupling incompressible flows with thin elastic structures undergoing finite deformations. The new AMP scheme is fully second-order accurate and stable, without sub-time-step iterations, even for very light structures when added-mass effects are strong. The fluid, governed by the incompressible Navier-Stokes equations, is solved in velocity-pressure form using a fractional-step method; large deformations are treated with a mixed Eulerian-Lagrangian approach on deforming composite grids. The motion of the thin structure is governed by a generalized Euler-Bernoulli beam model, and these equations are solved in a Lagrangian frame using two approaches, one based on finite differences and the other on finite elements. The key AMP interface condition is a generalized Robin (mixed) condition on the fluid pressure. This condition, which is derived at a continuous level, has no adjustable parameters and is applied at the discrete level to couple the partitioned domain solvers. Special treatment of the AMP condition is required to couple the finite-element beam solver with the finite-difference-based fluid solver, and two coupling approaches are described. A normal-mode stability analysis is performed for a linearized model problem involving a beam separating two fluid domains, and it is shown that the AMP scheme is stable independent of the ratio of the mass of the fluid to that of the structure. A traditional partitioned (TP) scheme using a Dirichlet-Neumann coupling for the same model problem is shown to be unconditionally unstable if the added mass of the fluid is too large. A series of benchmark problems of increasing complexity are considered to illustrate the behavior of the AMP algorithm, and to compare the behavior with that of the TP scheme. The results of all these benchmark problems verify the stability and accuracy of the AMP scheme. Results for one benchmark problem modeling blood flow in a deforming artery are also compared with corresponding results available in the literature.
\end{abstract}

Keywords: fluid-structure interaction; added-mass instability; incompressible fluid flow; moving overlapping grids; deformable bodies; Euler-Bernoulli beams

\footnotetext{
* Department of Mathematical Sciences, Rensselaer Polytechnic Institute, 110 8th Street, Troy, NY 12180, USA. Email addresses: lil19@rpi.edu (L. Li), henshw@rpi.edu (W. D. Henshaw), banksj3@rpi.edu (J. W. Banks), schwed@rpi.edu (D. W. Schwendeman), amain8511@gmail.com (G.A. Main)

${ }^{1}$ Research supported by the Margaret A. Darrin Postdoctoral Fellowship.

${ }^{2}$ This work was supported by contracts from the U.S. Department of Energy ASCR Applied Math Program.

${ }^{3}$ Research supported by the National Science Foundation under grant DMS-1519934.

${ }^{4}$ Research supported by a U.S. Presidential Early Career Award for Scientists and Engineers.

${ }^{5}$ Research supported by the U.S. Department of Energy NNSA Stewardship Science Graduate Fellowship.
} 


\section{Contents}

1 Introduction $\quad 3$

2 Governing equations $\quad 4$

3 Added-Mass Partitioning 6

3.1 Continuous AMP Interface Condition $\ldots \ldots \ldots \ldots \ldots$

3.2 Partitioned Schemes Using the AMP Condition . . . . . . . . . . . . . . . . 7

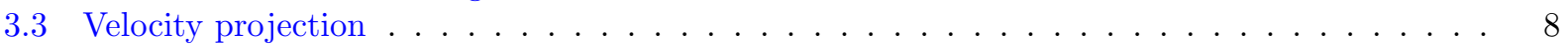

4 The AMP FSI time-stepping algorithm $r$

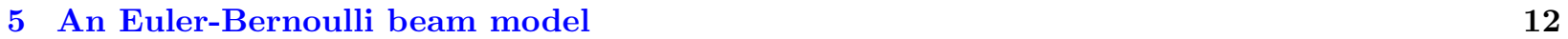

6 Analysis of a model problem for a two-dimensional beam 13

6.1 Exact solution of the continuous model problem . . . . . . . . . . . . . . . . . . . . 14

6.2 Stability analysis of the AMP and TP schemes . . . . . . . . . . . . . . 16

6.3 Pressure regularization for rigid-wall boundary conditions . . . . . . . . . . . . . . . 19

7 Numerical approach using deforming composite grids (DCG) 20

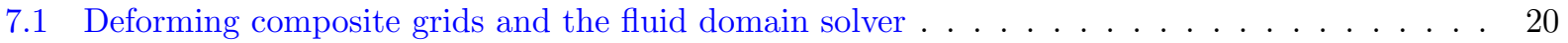

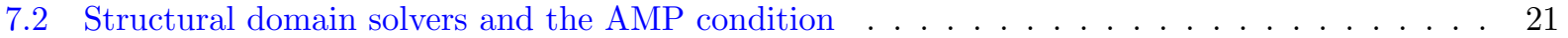

$7.2 .1 \quad$ AMP-PBA : Predict Beam Acceleration . . . . . . . . . . . . . . . . . 23

$7.2 .2 \quad$ AMP-PBF : Predict Beam internal Force . . . . . . . . . . . . . . . . . . . . . 23

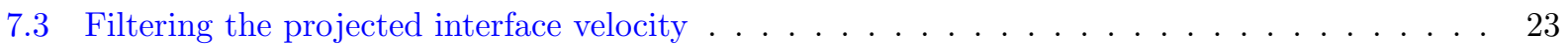

7.4 Time step determination . . . . . . . . . . . . . . . . . . . . . . . . 23

8 Numerical Results $\quad \mathbf{2 4}$

8.1 Verification results for a beam with negligible thickness $\ldots \ldots \ldots \ldots \ldots \ldots$

8.1 .1 Manufactured Solutions . . . . . . . . . . . . . . . . . . . . . . 24

8.1.2 Steady state beam bounding a pressurized fluid chamber . . . . . . . . . . . . 25

8.2 Verification results for a beam with finite thickness . . . . . . . . . . . . . 27

8.2.1 Dynamic motion of a flat beam separating two fluid chambers . . . . . . . . . . . . 28

8.2.2 Steady state beam separating two pressurized fluid chambers . . . . . . . . . . . . . 30

8.3 Traveling-wave pressure-pulse in an elastic tube . . . . . . . . . . . . . . . . . . 31

8.3 .1 Parameter Set I . . . . . . . . . . . . . . . . . . . . . . . . . . 32

8.3 .2 Parameter Set II . . . . . . . . . . . . . . . . . . . . . . . . . 35

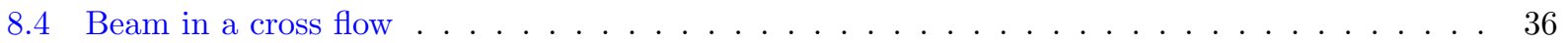

9 Conclusions $\quad 40$ 


\section{Introduction}

Fluid-structure interaction (FSI) problems that describe the motion of an incompressible fluid coupled to a thin walled structure (beam or shell) arise in many applications such as those in structural engineering and biomedicine. Such FSI problems are often modeled mathematically by suitable partial differential equations for the fluids and structures in their respective domains, together with matching conditions at the boundaries of the domains where the solutions of the equations interact. Numerical algorithms used to solve these FSI problems can be classified into two main categories. Algorithms belonging to one category, called monolithic schemes, treat the equations for the fluids and structures along with interface and boundary conditions as a large system of evolution equations, and then advance the solutions together. The other category of algorithms are partitioned schemes (also known as modular or sequential schemes), and these algorithms employ separate solvers for the fluids and structures which are coupled at the interface. Sub-iterations are often performed at each time step of partitioned algorithms for stability. Even though many existing partitioned schemes suffer from moderate to serious stability issues in certain problem regimes, they are often preferred since they can make use of existing solvers and can be more efficient than monolithic schemes.

The traditional partitioned algorithm for beams (or shells) uses the velocity and/or acceleration of the solid as a boundary condition on the fluid. The force of the fluid is accounted for through a body forcing on the beam. It has been found that partitioned schemes may be unstable, or require multiple sub-iterations per time step, when the density of the structure is similar to or lighter than that of the fluid $[1,2]$. These instabilities are attributed to the added-mass effect whereby the force required to accelerate a structure immersed in a fluid must also account for accelerating the surrounding fluid. The added-mass effect has been found to be especially problematic in many biological flows such as haemodynamics since the density of the fluid (blood) is similar to that of the adjacent structure (arterial walls) [3].

In our previous companion papers $[4,5]$, we developed stable added-mass partitioned (AMP) algorithms for linearized FSI problems involving incompressible Stokes fluids coupled to elastic bulk solids and to beams. The key ingredient of our approach is the use of non-traditional Robin interface conditions. These condition are derived at a continuous level, have no adjustable parameters, and are amenable for incorporation in high-order accurate schemes. Using mode analysis, the AMP approach was shown to be stable without subiterations per time step, and the numerical results demonstrated second-order accuracy in the max-norm. In the present paper, we focus on the case of thin structures and our primary purpose is to extend the AMP scheme in [5] to the case of finite amplitude motions in more general geometries. In particular, the AMP interface conditions are derived for beams of finite thickness, for beams with fluid on two sides, and for beams with a free end immersed in the fluid. Finite amplitude motions of the beam results in finite deformations of the surrounding fluid domain. Our numerical approach for the solution of the fluid equations in an evolving fluid domain is based on the use of deforming composite grids (DCG). The DCG approach for FSI problems was described first in [6] for inviscid compressible flow coupled to a linearly elastic solid, and later in [7] for compressible flow coupled to nonlinear hyperelastic solids. The approach is extended here for incompressible flow coupled to beams. Both finite-difference and finite-element approximations to the equations governing the motion of the beam are considered, and issues concerning the interface coupling of a finite-element based beam solver to a finite-difference based fluid solver are discussed. The stability analysis in [5] is also extended to treat beams with fluid on two sides. Finally, several benchmark FSI problems are presented to illustrate the stability and accuracy of the present AMP algorithm.

The investigation of FSI problems and the development of numerical approximations for their solution are very active areas of research, see for example [8-10] and the references therein. For the coupling of incompressible flows and bulk solids, the work in $[3,4,10-12]$ describes recent developments. The development of partitioned schemes for the case of incompressible fluids coupled to thin structures, the focus of this paper, is also an active area. The first stable partitioned scheme for incompressible flow coupled to thin structures was the "kinematically coupled scheme" of Guidoboni et al. [13] which uses an operator splitting of the kinematic interface condition (matching the fluid and beam velocities). This scheme was further advanced in $[14,15]$. Lukáčová-Medvid'ová et al. [16] developed a partitioned scheme based on the kinematically coupled scheme that uses Strang splitting. Fernandez and collaborators have also developed stable partitioned schemes for incompressible flow coupled to thin structures that are based on a time-splitting of the kinematic boundary condition [17-22]. However, it appears to be difficult to achieve higher than first-order accuracy with the time-splitting approach. The AMP approximation, in contrast, is not based on a time 
splitting and is amenable to second- or even higher-order accuracy. Second-order accuracy in the max-norm was demonstrated in [5] for linearized problems, while in this paper second-order accuracy is shown for the full nonlinear problem with deforming domains.

The remainder of the paper is organized as follows. In Section 2 we describe the governing equations. The AMP interface conditions are derived in Section 3. A second-order accurate predictor-corrector algorithm based on these conditions is described in Section 4. A specific choice for the beam model is given in Section 5 . In Section 6, the stability of the AMP scheme is shown for a linearized FSI problem involving a beam with fluid on two sides. The numerical approach used for moving domains based on deforming composite grids, our numerical approaches for the beam solver, and the treatment of the AMP interface conditions are described in Section 7. Numerical results presented in Section 8 carefully demonstrate the stability and accuracy of the AMP algorithm. Conclusions are provided in Section 9.

\section{Governing equations}

We consider the fluid-structure coupling of an incompressible fluid and a thin deformable structure. The fluid occupies the domain $\mathbf{x} \in \Omega(t)$ while the structure lies in the domain $\mathbf{x} \in \bar{\Omega}(t)$, where $\mathbf{x}$ is position and $t$ is time. The coupling of the fluid and structure occurs along the interface $\Gamma(t)$, see Figure 1. It is assumed that the fluid is governed by the incompressible Navier-Stokes equations, which in an Eulerian frame are given by

$$
\begin{array}{ll}
\frac{\partial \mathbf{v}}{\partial t}+(\mathbf{v} \cdot \nabla) \mathbf{v}=\frac{1}{\rho} \nabla \cdot \boldsymbol{\sigma}, & \mathbf{x} \in \Omega(t), \\
\nabla \cdot \mathbf{v}=0, & \mathbf{x} \in \Omega(t),
\end{array}
$$

where $\rho$ is the (constant) fluid density and $\mathbf{v}=\mathbf{v}(\mathbf{x}, t)$ is the fluid velocity. The fluid stress tensor, $\boldsymbol{\sigma}=\boldsymbol{\sigma}(\mathbf{x}, t)$, is given by

$$
\boldsymbol{\sigma}=-p \mathbf{I}+\boldsymbol{\tau}, \quad \boldsymbol{\tau}=\mu\left[\nabla \mathbf{v}+(\nabla \mathbf{v})^{T}\right],
$$

where $p=p(\mathbf{x}, t)$ is the pressure, $\mathbf{I}$ is the identity tensor, $\boldsymbol{\tau}$ is the viscous stress tensor, and $\mu$ is the (constant) fluid viscosity. For future reference, the components of a vector such as $\mathbf{v}$ will be denoted by $v_{m}, m=1,2,3$ (i.e. $\mathbf{v}=\left[v_{1}, v_{2}, v_{3}\right]^{T}$ ), while components of a tensor such as $\boldsymbol{\sigma}$ will be denoted by $\sigma_{m n}, m, n=1,2,3$. The velocity-divergence form of the equations given by (1) and (2) require appropriate initial and boundary conditions, as well as conditions on $\Gamma(t)$ where the behavior of the fluid is coupled to that of the solid (as discussed below).

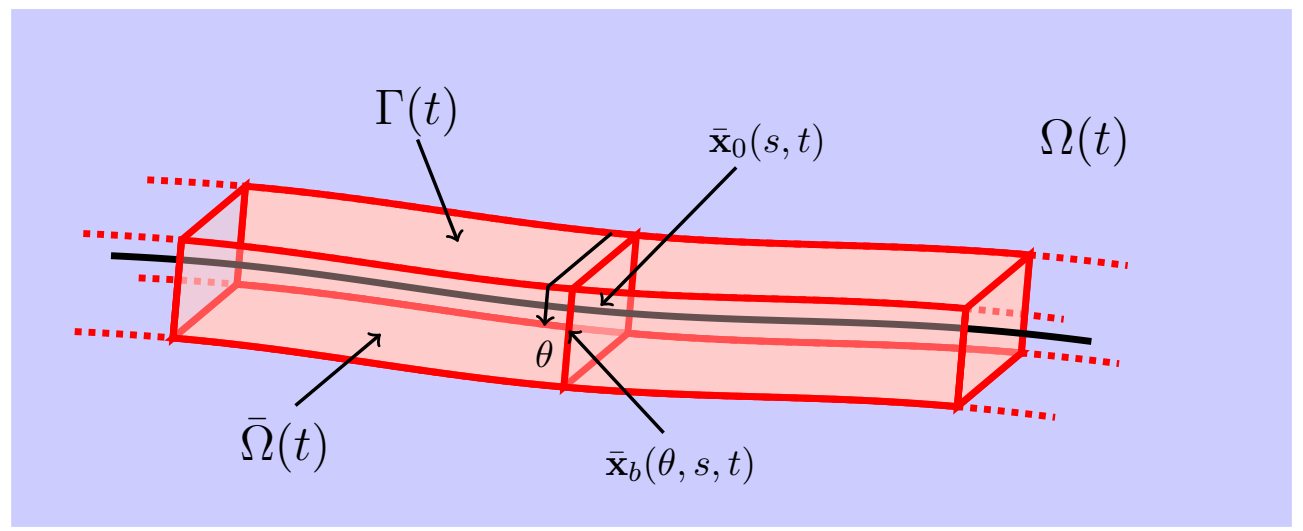

Figure 1: Geometry of a three-dimensional beam immersed in a fluid. The solid domain is $\bar{\Omega}(t)$. The fluid domain is $\Omega(t)$. The interface between the fluid and solid is $\Gamma(t)$. The beam reference-line is $\overline{\mathbf{x}}_{0}(s, t)$. A point on the surface of the beam is denoted by $\overline{\mathbf{x}}_{b}(\theta, s, t)$ where $\theta \in \mathcal{P}(s)$ is the parameterization of the cross-sectional surface curve. The fluid tractions on the surface points $\overline{\mathbf{x}}_{b}(\theta, s, t)$, for $\theta \in \mathcal{P}(s)$, contribute to the force on the beam at $\overline{\mathbf{x}}_{0}(s, t)$. 
An elliptic equation for the fluid pressure can be derived from (1)-(3) as

$$
\Delta p=-\rho \nabla \mathbf{v}:(\nabla \mathbf{v})^{T}, \quad \mathbf{x} \in \Omega(t),
$$

where

$$
\nabla \mathbf{v}:(\nabla \mathbf{v})^{T} \equiv \sum_{i=1}^{3} \sum_{j=1}^{3} \frac{\partial v_{i}}{\partial x_{j}} \frac{\partial v_{j}}{\partial x_{i}} .
$$

In the velocity-pressure form of the incompressible Navier-Stokes equations, the Poisson equation (4) is used in place of (2). This form requires an additional boundary condition, and a natural choice is $\nabla \cdot \mathbf{v}=0$ for $\mathbf{x} \in \partial \Omega(t)$, see [23] for example.

The structure is assumed to be thin and governed by a beam model, which describes the evolution of the displacement, $\overline{\mathbf{u}}=\overline{\mathbf{u}}(s, t)$, of a reference centerline curve of the beam as a function of a Lagrangian coordinate $s \in \overline{\mathcal{R}}$ and time $t$. (Overbars are used throughout to denote quantities belonging to the structure.) The beam model has the form

$$
\bar{\rho} \bar{A} \frac{\partial^{2} \overline{\mathbf{u}}}{\partial t^{2}}=\overline{\mathbf{L}}(\overline{\mathbf{u}}, \overline{\mathbf{v}})+\overline{\mathbf{f}}(s, t), \quad s \in \overline{\mathcal{R}},
$$

where $\bar{\rho}$ is the (constant) beam density, $\bar{A}=\bar{A}(s)$ is the cross-sectional area of the beam, $\overline{\mathbf{L}}$ is the internal restoring force per unit length which depends on $\overline{\mathbf{u}}$ and on the velocity, $\overline{\mathbf{v}}=\partial \overline{\mathbf{u}} / \partial t$, of the reference curve (in general), and $\overline{\mathbf{f}}$ is the external force per unit length on the beam from the fluid (defined below). The effects of torsion are neglected in the present beam model. In physical space, the position $\overline{\mathbf{x}}_{0}$ of the reference curve is given by

$$
\overline{\mathbf{x}}_{0}(s, t)=\overline{\mathbf{x}}_{0}(s, 0)+\overline{\mathbf{u}}(s, t), \quad s \in \overline{\mathcal{R}},
$$

where $\overline{\mathbf{x}}_{0}(s, 0)$ is the initial position of the reference curve of the beam. The position $\overline{\mathbf{x}}_{b}$ of the surface of the beam is given by

$$
\overline{\mathbf{x}}_{b}(\theta, s, t)=\overline{\mathbf{x}}_{0}(s, t)+\overline{\mathbf{z}}(\theta, s, t), \quad \theta \in \overline{\mathcal{P}}, \quad s \in \overline{\mathcal{R}},
$$

where $\overline{\mathbf{z}}$ is the offset from the reference curve to the perimeter $\overline{\mathcal{P}}=\overline{\mathcal{P}}(s)$ of the cross section of the beam at $s$, parameterized by arclength $\theta$ along the perimeter. Note that $\overline{\mathbf{z}}$ is defined in terms of the beam variables; its form for an Euler-Bernoulli beam is given in Section 5. The surface of the beam given by (7) defines the position of the fluid-structure interface $\Gamma(t)$ (see Figure 1).

At the fluid-structure interface, the velocity of the fluid at a point on the surface of the beam matches the corresponding velocity of the beam surface,

$$
\mathbf{v}\left(\overline{\mathbf{x}}_{b}(\theta, s, t), t\right)=\overline{\mathbf{v}}_{b}(\theta, s, t), \quad \theta \in \overline{\mathcal{P}}, \quad s \in \overline{\mathcal{R}},
$$

where

$$
\overline{\mathbf{v}}_{b}(\theta, s, t)=\frac{\partial}{\partial t} \overline{\mathbf{x}}_{b}(\theta, s, t)=\overline{\mathbf{v}}(s, t)+\overline{\mathbf{w}}(\theta, s, t), \quad \overline{\mathbf{w}}(\theta, s, t)=\frac{\partial}{\partial t} \overline{\mathbf{z}}(\theta, s, t) .
$$

Formulas for the finite-thickness corrections, $\overline{\mathbf{z}}$ and $\overline{\mathbf{w}}$, are given in Section 5 for the case of an Euler-Bernoulli beam model. The dynamic matching condition (balance of forces) defines the force per unit length on the beam, $\overline{\mathbf{f}}$, in (5) in terms of the fluid force on the surface of the beam,

$$
\overline{\mathbf{f}}(s, t)=-\int_{\overline{\mathcal{P}}}(\boldsymbol{\sigma} \mathbf{n})(\hat{\theta}, s, t) d \hat{\theta}, \quad s \in \overline{\mathcal{R}},
$$

where

$$
(\boldsymbol{\sigma n})(\theta, s, t) \equiv \boldsymbol{\sigma}\left(\overline{\mathbf{x}}_{b}(\theta, s, t), t\right) \mathbf{n}\left(\overline{\mathbf{x}}_{b}(\theta, s, t), t\right) .
$$

Here, $\mathbf{n}$ denotes the unit normal to the beam surface (outward pointing from the fluid domain).

We note that for the case of a beam in two dimensions as shown in Figure 2, the surface position in (7) reduces to

$$
\overline{\mathbf{x}}_{b, \pm}(s, t)=\overline{\mathbf{x}}_{0}(s, t)+\overline{\mathbf{z}}_{ \pm}(s, t), \quad s \in \overline{\mathcal{R}}
$$




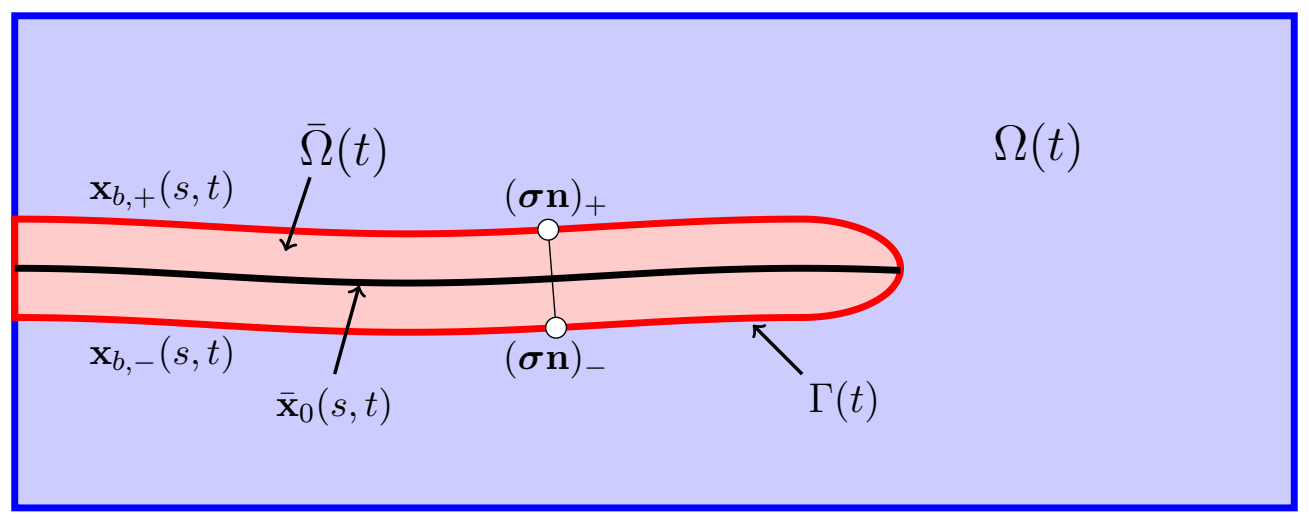

Figure 2: Geometry of a two-dimensional beam immersed in a fluid. The beam reference surface is $\overline{\mathbf{x}}_{0}(s, t)$. The top and bottom surfaces of the beam are the $\overline{\mathbf{x}}_{b,+}(s, t)$ and $\overline{\mathbf{x}}_{b,-}(s, t)$, respectively.

where the plus and minus signs denote the upper and lower surfaces of the beam, respectively. The velocity on the surfaces of the beam are now given by

$$
\overline{\mathbf{v}}_{b, \pm}(s, t)=\frac{\partial}{\partial t} \overline{\mathbf{x}}_{b, \pm}(s, t)=\overline{\mathbf{v}}(s, t)+\overline{\mathbf{w}}_{ \pm}(s, t), \quad \overline{\mathbf{w}}_{ \pm}(s, t)=\frac{\partial}{\partial t} \overline{\mathbf{z}}_{ \pm}(s, t),
$$

and if fluid regions exist on both surfaces of the beam, then the the external force is given by

$$
\overline{\mathbf{f}}(s, t)=\left[(\boldsymbol{\sigma} \mathbf{n})_{+}(s, t)+(\boldsymbol{\sigma} \mathbf{n})_{-}(s, t)\right], \quad s \in \overline{\mathcal{R}},
$$

where

$$
(\boldsymbol{\sigma} \mathbf{n})_{ \pm}(s, t) \equiv \boldsymbol{\sigma}\left(\overline{\mathbf{x}}_{b, \pm}(s, t), t\right) \mathbf{n}\left(\overline{\mathbf{x}}_{b, \pm}(s, t), t\right) .
$$

The formulas (12)-(15) also hold for the case of a thin plate or shell provided the dependent variables, $\overline{\mathbf{x}}_{0}(\mathbf{s}, t)$, $\overline{\mathbf{v}}(\mathbf{s}, t)$, etc., are taken to depend on two independent parameters $\left(s_{1}, s_{2}\right)=\mathbf{s} \in \mathbb{R}^{2}$ that parameterize the reference surface of the shell.

\section{Added-Mass Partitioning}

In the traditional approach to partitioned fluid-structure interaction, the kinematic condition (8) is satisfied by imposing the motion of the solid as an interface condition on the fluid. This inherently one-sided approach is one root cause leading to added-mass instabilities for partitioned fluid-structure interaction. Various avenues to more symmetric imposition of (8) have been taken with various degrees of success. Here we pursue the Added Mass Partitioned (AMP) approach to coupling. The core AMP algorithm for coupling a linear incompressible fluid with a thin shell or beam was described in [5]. In that work a simplified model was considered where linearized equations of motion were used to describe infinitesimal amplitude perturbations from the specific geometry of fluid in a box with a beam on one of the faces of the box. This manuscript considers the more general case of the nonlinear Navier-Stokes equations with finite amplitude deformations applied to more general geometries.

\subsection{Continuous AMP Interface Condition}

The AMP interface condition can be derived at a continuous level by first taking the time derivative of the kinematic interface condition (8) and using the definition of the velocity on the surface of the beam (9) to give

$$
\begin{aligned}
\frac{D}{D t} \mathbf{v}\left(\overline{\mathbf{x}}_{b}(\theta, s, t), t\right) & =\frac{\partial}{\partial t} \overline{\mathbf{v}}(s, t)+\frac{\partial}{\partial t} \overline{\mathbf{w}}(\theta, s, t) \\
& =\frac{\partial^{2} \overline{\mathbf{u}}}{\partial t^{2}}(s, t)+\frac{\partial}{\partial t} \overline{\mathbf{w}}(\theta, s, t),
\end{aligned}
$$


where $D / D t=\partial_{t}+\mathbf{v} \cdot \nabla$ is the material derivative. The fluid acceleration, $D \mathbf{v} / D t$, is given by the fluid momentum equation (1), while the acceleration of the structure, $\partial^{2} \overline{\mathbf{u}} / \partial t^{2}$, is given by the beam equation (5). Substituting these definitions into (16) gives

$$
\frac{\bar{\rho} \bar{A}}{\rho} \nabla \cdot \boldsymbol{\sigma}\left(\overline{\mathbf{x}}_{b}(\theta, s, t), t\right)=\overline{\mathbf{L}}(\overline{\mathbf{u}}, \overline{\mathbf{v}})+\bar{\rho} \bar{A} \frac{\partial}{\partial t} \overline{\mathbf{w}}(\theta, s, t)+\overline{\mathbf{f}}(s, t), \quad \theta \in \overline{\mathcal{P}}, \quad s \in \overline{\mathcal{R}} .
$$

Finally, using (10) for the force $\overline{\mathbf{f}}$ yields the continuous compatibility interface condition

$$
\int_{\overline{\mathcal{P}}}(\boldsymbol{\sigma} \mathbf{n})(\hat{\theta}, s, t) d \hat{\theta}+\frac{\bar{\rho} \bar{A}}{\rho} \nabla \cdot \boldsymbol{\sigma}\left(\overline{\mathbf{x}}_{b}(\theta, s, t), t\right)=\overline{\mathbf{L}}(\overline{\mathbf{u}}, \overline{\mathbf{v}})+\bar{\rho} \bar{A} \frac{\partial}{\partial t} \overline{\mathbf{w}}(\theta, s, t), \quad \theta \in \overline{\mathcal{P}}, \quad s \in \overline{\mathcal{R}},
$$

which can be interpreted as a mixed Robin-type condition along the surface of the beam coupling the behaviors of the fluid and structure.

\subsection{Partitioned Schemes Using the AMP Condition}

Following the approach used in [5], we employ an AMP interface condition based on the compatibility condition (18) as a stable condition for partitioned schemes coupling incompressible fluids and thin structural beams. Equation (18) appears to be a condition that fully couples the fluid and solid values on the interface. However, a critical observation made in [5] is that (18) can be used as a stable condition for partitioned schemes by using predicted values for the solid variables $\overline{\mathbf{u}}^{(p)}, \overline{\mathbf{v}}^{(p)}$ and $\overline{\mathbf{w}}^{(p)}$ when evaluating the right-handside of (18). These predicted values for the solid are obtained in a first step of the partitioned scheme and the interface condition then becomes a mixed Robin-type condition for the fluid in a subsequent step of the scheme. This leads to the primary AMP interface condition:

Condition 1. The AMP interface condition for the fluid is

$$
\int_{\overline{\mathcal{P}}}(\boldsymbol{\sigma} \mathbf{n})(\hat{\theta}, s, t) d \hat{\theta}+\frac{\bar{\rho} \bar{A}}{\rho} \nabla \cdot \boldsymbol{\sigma}\left(\overline{\mathbf{x}}_{b}(\theta, s, t), t\right)=\overline{\mathbf{L}}^{(p)}+\bar{\rho} \bar{A} \frac{\partial}{\partial t} \overline{\mathbf{w}}^{(p)}(\theta, s, t), \quad \theta \in \overline{\mathcal{P}}, \quad s \in \overline{\mathcal{R}},
$$

where $\overline{\mathbf{u}}^{(p)}, \overline{\mathbf{v}}^{(p)}$ and $\overline{\mathbf{w}}^{(p)}$ are predicted solid variables, and $\overline{\mathbf{L}}^{(p)} \equiv \overline{\mathbf{L}}\left(\overline{\mathbf{u}}^{(p)}, \overline{\mathbf{v}}^{(p)}\right)$.

The essential philosophy in this approach is to embed the local behavior of solutions of the FSI problem to define the interface dynamics. Therefore, in a partitioned scheme the solid would be advanced first to a new time step, providing the predicted values used in the interface conditions (19) for the subsequent fluid solve. This process can be viewed as computing the solution of a generalized fluid-solid Riemann problem at the interface, thus extending the approach used in the AMP scheme for elastic solids coupled to inviscid compressible fluids discussed in $[6,7,24]$. In these previous studies, the FSI problems were purely hyperbolic and the fluid-solid Riemann problem involved local, piecewise constant states of the fluid and solid on either side of the interface. The solution was then computed using the method of characteristics, giving interface values that are impedance-weighted averages of the fluid and solid states. In the present case, involving an incompressible fluid, the initial states for the solid domain are $\overline{\mathbf{u}}^{(p)}, \overline{\mathbf{v}}^{(p)}$ and $\overline{\mathbf{w}}^{(p)}$, while the solution of the generalized fluid-solid Riemann problem requires the solution of an elliptic problem in the entire fluid domain with (19) providing the appropriate condition along the interface.

If a fractional-step scheme is used to integrate the fluid equations, with the velocity and pressure being updated in separate stages, then suitable boundary conditions can be derived by decomposing (19) into normal and tangential components. The normal component gives a boundary condition for the pressure, while the tangential components, along with the continuity equation (2), provide boundary conditions for the velocity.

Condition 2. The AMP interface condition for the pressure equation (4) is given by

$$
\mathbf{n}^{T} \int_{\overline{\mathcal{P}}}(p \mathbf{n})(\hat{\theta}, s, t) d \hat{\theta}+\frac{\bar{\rho} \bar{A}}{\rho} \frac{\partial p}{\partial n}=\mathbf{n}^{T}\left[-\overline{\mathbf{L}}^{(p)}-\bar{\rho} \bar{A} \frac{\partial}{\partial t} \overline{\mathbf{w}}^{(p)}+\frac{\mu \bar{\rho} \bar{A}}{\rho} \Delta \mathbf{v}+\int_{\overline{\mathcal{P}}}(\boldsymbol{\tau} \mathbf{n})(\hat{\theta}, s, t) d \hat{\theta}\right],
$$

for $\theta \in \overline{\mathcal{P}}$ and $s \in \overline{\mathcal{R}}$, where

$$
(p \mathbf{n})(\theta, s, t) \equiv p\left(\overline{\mathbf{x}}_{b}(\theta, s, t), t\right) \mathbf{n}\left(\overline{\mathbf{x}}_{b}(\theta, s, t), t\right), \quad(\boldsymbol{\tau} \mathbf{n})(\theta, s, t) \equiv \boldsymbol{\tau}\left(\overline{\mathbf{x}}_{b}(\theta, s, t), t\right) \mathbf{n}\left(\overline{\mathbf{x}}_{b}(\theta, s, t), t\right) .
$$


Condition 3. The AMP interface conditions for the velocity when integrating the momentum equation (1) are given by

$$
\mathbf{t}_{m}^{T}\left[\int_{\overline{\mathcal{P}}}(\boldsymbol{\tau} \mathbf{n})(\hat{\theta}, s, t) d \hat{\theta}+\frac{\mu \bar{\rho} \bar{A}}{\rho} \Delta \mathbf{v}\right]=\mathbf{t}_{m}^{T}\left[\frac{\bar{\rho} \bar{A}}{\rho} \nabla p+\overline{\mathbf{L}}^{(p)}+\bar{\rho} \bar{A} \frac{\partial}{\partial t} \overline{\mathbf{w}}^{(p)}+\int_{\overline{\mathcal{P}}}(p \mathbf{n})(\hat{\theta}, s, t) d \hat{\theta}\right],
$$

for $\theta \in \overline{\mathcal{P}}$ and $s \in \overline{\mathcal{R}}$, where $\mathbf{t}_{m}=\mathbf{t}_{m}(\theta, s, t), m=1,2$, denote mutually orthonormal tangent vectors.

We note that if the beam motion only depends on one degree of freedom (e.g. as with the Euler-Bernoulli beam model described in Section 5) then only (20) is used and (21) should be replaced with a standard condition on the tangential velocity. For example, (21) can be replaced by setting the tangential components of the fluid velocity equal to the tangential components of the velocity on the beam surface, i.e. a no-slip type condition on the tangential velocity,

$$
\mathbf{t}_{m}^{T} \mathbf{v}\left(\overline{\mathbf{x}}_{b}(\theta, s, t), t\right)=\mathbf{t}_{m}^{T} \overline{\mathbf{v}}(s, t)+\mathbf{t}_{m}^{T} \overline{\mathbf{w}}(\theta, s, t), \quad m=1,2,
$$

for $\theta \in \overline{\mathcal{P}}$ and $s \in \overline{\mathcal{R}}$. Another choice for the boundary condition could be a slip wall condition which sets the tangential components of the traction to zero,

$$
\mathbf{t}_{m}^{T}(\boldsymbol{\tau} \mathbf{n})(\theta, s, t)=0, \quad m=1,2
$$

for $\theta \in \overline{\mathcal{P}}$ and $s \in \overline{\mathcal{R}}$.

\subsection{Velocity projection}

The velocity at the interface in the AMP scheme is defined to be a weighted average of the predicted velocities from the fluid and solid. This averaging can be motivated by noting that for heavy beams the motion of the interface is primarily determined from the beam and thus the interface velocity should be nearly equal to that of the structure. For very light beams, on the other hand, the fluid interface approaches a free surface where the motion of the interface is primarily determined by the fluid itself. The weighted average given in [5] correctly accounts for these two limits, and this average is extended here to account for beams of finite thickness.

\section{Velocity Projection Algorithm.}

1. Compute the projected surface velocity $\mathbf{v}^{I}(\theta, s, t)$ on the surface of the beam as a weighted average of the predicted surface velocities of the fluid $\mathbf{v}_{b}^{(p)}(\theta, s, t)$ and the beam $\overline{\mathbf{v}}_{b}^{(p)}(\theta, s, t)$,

$$
\mathbf{v}^{I}(\theta, s, t)=\gamma \mathbf{v}_{b}^{(p)}(\theta, s, t)+(1-\gamma) \overline{\mathbf{v}}_{b}^{(p)}(\theta, s, t), \quad \theta \in \overline{\mathcal{P}}, \quad s \in \overline{\mathcal{R}},
$$

where

$$
\gamma=\frac{1}{1+(\bar{\rho} \bar{A}) /\left(\rho A_{f}\right)} .
$$

Here, $A_{f}=A_{f}(s, t)$ is the area of a characteristic annular region in the fluid surrounding the beam at a position $\overline{\mathbf{x}}_{0}(s, t)$ on the reference curve. It has been found that the algorithm is insensitive to the choice of this characteristic area [5]. For the numerical examples in Section 8, where the domains are $\mathcal{O}(1)$ in size, we have simply chosen $A_{f}=1$ in all cases.

2. Transfer the interface velocities $\mathbf{v}^{I}$ (minus the surface correction $\overline{\mathbf{w}}$ ) onto the beam reference curve using an average of the values on the cross-section perimeter curve $\overline{\mathcal{P}}$

$$
\overline{\mathbf{v}}_{0}(s, t)=\frac{1}{|\overline{\mathcal{P}}|} \int_{\overline{\mathcal{P}}}\left(\mathbf{v}^{I}(\hat{\theta}, s, t)-\overline{\mathbf{w}}(\hat{\theta}, s, t)\right) d \hat{\theta}, \quad s \in \overline{\mathcal{R}},
$$

where $|\overline{\mathcal{P}}|$ denotes the length of the perimeter curve $\overline{\mathcal{P}}$. We note that if the fluid density is not constant, or if the beam separates two fluid regions in two dimensions, then the average in (26) could be replaced by a density-weighted average. Redefine the reference-curve velocity $\overline{\mathbf{v}}(s, t)$ to equal $\overline{\mathbf{v}}_{0}(s, t)$. 
3. Set the fluid velocity on the surface of the beam to be

$$
\mathbf{v}\left(\overline{\mathbf{x}}_{b}(\theta, s, t), t\right)=\overline{\mathbf{v}}(s, t)+\overline{\mathbf{w}}(\theta, s, t), \quad \theta \in \overline{\mathcal{P}}, \quad s \in \overline{\mathcal{R}},
$$

following the kinematic matching conditions in (8) and (9) so that the beam reference-curve velocity provides a consistent definition of the fluid surface velocity.

From the perspective of numerical stability, this velocity projection appears to be needed only when the beam is quite light. Otherwise the fluid velocity on the interface could be taken as the velocity of the beam. This observation is supported by the linear stability analysis in [5] as well as numerical experiments. However, in practice we find that the velocity projection is never harmful and we therefore advocate its use. In addition, for very light beams the interface acts as a free surface with no surface tension (assuming $\overline{\mathbf{L}}$ is also very small). In this case, the fluid interface velocity is smoothed using a few steps of a fourth-order filter to remove oscillations that can be excited on the interface, see Section 7.3 for more details. Finally note that for cases where the beam supports motion only in the normal direction to the beam, only the normal component of the fluid velocity is projected, i.e.

$$
\left(\mathbf{n}^{T} \mathbf{v}\right)^{I}(\theta, s, t)=\gamma\left(\mathbf{n}^{T} \mathbf{v}_{b}^{(p)}\right)(\theta, s, t)+(1-\gamma)\left(\mathbf{n}^{T} \overline{\mathbf{v}}_{b}^{(p)}\right)(\theta, s, t), \quad \theta \in \overline{\mathcal{P}}, \quad s \in \overline{\mathcal{R}},
$$

where $\gamma$ is given by $(25)$ as before.

\section{The AMP FSI time-stepping algorithm}

In this section we outline the FSI time-stepping algorithm, including deforming geometry, for an incompressible fluid governed by the Navier-Stokes equations and a thin structure determined by the general beam model in (5). The intent is to provide a concrete description of the AMP algorithm with sufficient details to indicate the primary extensions required from the original scheme described in [5]. The fluid equations are solved in velocity-pressure form using a second-order accurate fractional-step algorithm [5, 23, 25]. The beam equations are advanced with a second-order accurate predictor-corrector scheme. In practice we have also used a predictor-corrector version of the Newmark-beta scheme for the beam equations.

Given the discrete solution at the current time $t^{n}$, and one previous time level $t^{n-1}$, the goal of the FSI algorithm is to determine the solution at time $t^{n+1}$. The algorithm is written for a fixed time-step, $\Delta t$, so that $t^{n}=n \Delta t$, but is easily extended to a variable $\Delta t$. A predictor-corrector scheme that implements the FSI algorithm is as follows:

\section{Begin predictor.}

Stage I - structure: Predicted values for the displacement and velocity of the shell are obtained using a second-order accurate leap-frog scheme given by

$$
\begin{array}{clrl}
\frac{\overline{\mathbf{u}}_{\mathbf{j}}^{(p)}-\overline{\mathbf{u}}_{\mathbf{j}}^{n-1}}{2 \Delta t} & =\overline{\mathbf{v}}_{\mathbf{j}}^{n}, & \mathbf{j} \in \overline{\mathcal{R}}_{h}, \\
\bar{\rho} \bar{A}_{\mathbf{j}} \frac{\overline{\mathbf{v}}_{\mathbf{j}}^{(p)}-\overline{\mathbf{v}}_{\mathbf{j}}^{n-1}}{2 \Delta t}=\overline{\mathbf{L}}_{h}\left(\overline{\mathbf{u}}_{\mathbf{j}}^{n}, \overline{\mathbf{v}}_{\mathbf{j}}^{n}\right)+\overline{\mathbf{f}}_{\mathbf{j}}^{n}, & \mathbf{j} \in \overline{\mathcal{R}}_{h},
\end{array}
$$

where the external force at $t=t^{n}$ is given in terms of the fluid stress on the surface of the beam by a discrete approximation to (10),

$$
\overline{\mathbf{f}}_{\mathbf{j}}^{n}=\sum_{\mathbf{k} \in \overline{\mathcal{P}}_{h, \mathbf{j}}}(\boldsymbol{\sigma} \mathbf{n})_{\mathbf{k}}^{n} \Delta \theta
$$

Here $\mathbf{j} \in \overline{\mathcal{R}}_{h}$ denotes the indices of the discrete points along the beam reference curve and $\mathbf{i} \in \Gamma_{h}$ denotes the corresponding indices of the discrete fluid points on the beam surface. There is a cross section of the beam identified for each point $\mathbf{j}$ along the reference curve, and the points $\mathbf{k} \in \overline{\mathcal{P}}_{h, \mathbf{j}}$ denote the discrete representation of the curve on the perimeter of the cross section. 
Stage II - grid generation: Predicted values for the deformed interface positions are obtained based on the predicted reference-curve deformation using discrete versions of the formulas in (6) and (7). The predicted fluid grid $\mathcal{G}^{(p)}$ is then defined in terms of the interface positions using an appropriate mapping. In the context of overlapping grids (see Section 7.1) an interface-fitted fluid grid is generated with a hyperbolic marching algorithm and then a new overlapping grid is generated.

Stage III - fluid velocity: Predicted values for the fluid velocity are obtained using a second-order accurate Adams-Bashforth scheme

$$
\rho \frac{\mathbf{v}_{\mathbf{i}}^{(p)}-\mathbf{v}_{\mathbf{i}}^{n}}{\Delta t}=\frac{3}{2} \mathbf{F}_{\mathbf{i}}^{n}-\frac{1}{2} \mathbf{F}_{\mathbf{i}}^{n-1}, \quad \mathbf{i} \in \Omega_{h},
$$

where

$$
\mathbf{F}_{\mathbf{i}}^{n} \equiv-\left(\left(\mathbf{v}_{\mathbf{i}}^{n}-\mathbf{w}_{\mathbf{i}}^{n}\right) \cdot \nabla\right) \mathbf{v}_{\mathbf{i}}^{n}-\nabla_{h} p_{\mathbf{i}}^{n}+\mu \Delta_{h} \mathbf{v}_{\mathbf{i}}^{n} .
$$

On moving grids the equations are solved in a moving coordinate system (see Section 7.1) and $\mathbf{w}_{\mathbf{i}}^{n}$ is the grid velocity. From (21), the boundary conditions for $\mathbf{v}_{\mathbf{i}}^{(p)}$ on $\Gamma_{h}$ are

$$
\left.\begin{array}{c}
\mathbf{t}_{m, \mathbf{i}}^{T}\left[\sum_{\mathbf{k} \in \overline{\mathcal{P}}_{h, \mathbf{j}}} \boldsymbol{\tau}_{\mathbf{k}}^{(p)} \mathbf{n}_{\mathbf{k}} \Delta \theta+\frac{\mu \bar{\rho} \bar{A}_{\mathbf{j}}}{\rho} \Delta_{h} \mathbf{v}_{\mathbf{i}}^{(p)}\right]=\mathbf{t}_{m, \mathbf{i}}^{T}\left[\frac{\bar{\rho} \bar{A}_{\mathbf{j}}}{\rho} \nabla_{h} p_{\mathbf{i}}^{(p)}+\bar{H}_{\mathbf{j}}^{(p)}+\sum_{\mathbf{k} \in \overline{\mathcal{P}}_{h, \mathbf{j}}} p_{\mathbf{k}}^{(p)} \mathbf{n}_{\mathbf{k}} \Delta \theta\right], \\
\nabla_{h} \cdot \mathbf{v}_{\mathbf{i}}^{(p)}=0,
\end{array}\right\}
$$

where $\mathbf{i} \in \Gamma_{h}, \mathbf{j} \in \overline{\mathcal{R}}_{h}, m=1,2$, and

$$
\boldsymbol{\tau}_{\mathbf{i}}^{(p)}=\mu\left[\nabla_{h} \mathbf{v}_{\mathbf{i}}^{(p)}+\left(\nabla_{h} \mathbf{v}_{\mathbf{i}}^{(p)}\right)^{T}\right], \quad \bar{H}_{\mathbf{j}}^{(p)}=\overline{\mathbf{L}}_{h}\left(\overline{\mathbf{u}}_{\mathbf{j}}^{(p)}, \overline{\mathbf{v}}_{\mathbf{j}}^{(p)}\right)+\bar{\rho} \bar{A}_{\mathbf{j}} \dot{\overline{\mathbf{w}}}_{\mathbf{j}}^{(p)} .
$$

The term $\dot{\overline{\mathbf{w}}}_{\mathbf{j}}^{(p)}$ in (32) is a predicted value for $\partial \overline{\mathbf{w}} / \partial t$, which for an Euler-Bernoulli beam can be computed from the predicted beam position, velocity and acceleration. At this stage of the time-stepping algorithm, a predicted value for the pressure, $p_{\mathbf{i}}^{(p)}$, on the right-hand side of (31) is not yet available. However, a sufficiently accurate value for the purposes of the boundary conditions can be obtained using a thirdorder extrapolation in time, $p_{\mathbf{i}}^{(p)}=3 p_{\mathbf{i}}^{n}-3 p_{\mathbf{i}}^{n-1}+p_{\mathbf{i}}^{n-2}$. This extrapolated pressure is replaced in the next stage of the algorithm by solving a discrete Poisson problem. Appropriate boundary conditions for $\mathbf{v}_{\mathbf{i}}^{(p)}$ should also be applied on other boundaries.

Stage IV - fluid pressure: Predicted values for the pressure are determined by solving the pressure equation

$$
\Delta_{h} p_{\mathbf{i}}^{(p)}=-\rho \nabla \mathbf{v}_{\mathbf{i}}^{(p)}:\left(\nabla \mathbf{v}_{\mathbf{i}}^{(p)}\right)^{T}, \quad \mathbf{i} \in \Omega_{h},
$$

subject to the boundary conditions,

$$
\mathbf{n}_{\mathbf{i}}^{T}\left[\sum_{\mathbf{k} \in \overline{\mathcal{P}}_{h, \mathbf{j}}} p_{\mathbf{k}}^{(p)} \mathbf{n}_{\mathbf{k}} \Delta \theta+\frac{\bar{\rho} \bar{A}_{\mathbf{j}}}{\rho} \nabla_{h} p_{\mathbf{i}}^{(p)}\right]=\mathbf{n}_{\mathbf{i}}^{T}\left[-\bar{H}_{\mathbf{j}}^{(p)}+\frac{\mu \bar{\rho} \bar{A}_{\mathbf{j}}}{\rho} \Delta_{h} \mathbf{v}_{\mathbf{i}}^{(p)}+\sum_{\mathbf{k} \in \overline{\mathcal{P}}_{h, \mathbf{j}}} \boldsymbol{\tau}_{\mathbf{k}}^{(p)} \mathbf{n}_{\mathbf{k}} \Delta \theta\right], \quad \mathbf{i} \in \Gamma_{h},
$$

with $\mathbf{j} \in \overline{\mathcal{R}}_{h}$ and appropriate conditions for $p_{\mathbf{i}}^{(p)}$ applied on the other boundaries. Note that in practice it is useful to add a divergence damping term to the right-hand side of the the pressure equation (33) following $[23,25]$.

\section{End predictor.}

\section{Begin corrector.}


Stage V - structure: Corrected values for the displacement and velocity of the shell are obtained using a second-order accurate Adams-Moulton scheme,

$$
\begin{array}{clrl}
\frac{\overline{\mathbf{u}}_{\mathbf{j}}^{n+1}-\overline{\mathbf{u}}_{\mathbf{j}}^{n}}{\Delta t} & =\overline{\mathbf{v}}_{\mathbf{j}}^{n+\frac{1}{2}}, & \mathbf{j} \in \overline{\mathcal{R}}_{h}, \\
\bar{\rho} \bar{A}_{\mathbf{j}} \frac{\overline{\mathbf{v}}_{\mathbf{j}}^{n+1}-\overline{\mathbf{v}}_{\mathbf{j}}^{n}}{\Delta t} & =\overline{\mathbf{L}}_{h}\left(\overline{\mathbf{u}}_{\mathbf{j}}^{n+\frac{1}{2}}, \overline{\mathbf{v}}_{\mathbf{j}}^{n+\frac{1}{2}}\right)+\overline{\mathbf{f}}_{\mathbf{j}}^{n+\frac{1}{2}}, & \mathbf{j} \in \overline{\mathcal{R}}_{h},
\end{array}
$$

where

$$
\overline{\mathbf{u}}_{\mathbf{j}}^{n+\frac{1}{2}} \equiv \frac{1}{2}\left(\overline{\mathbf{u}}_{\mathbf{j}}^{(p)}+\overline{\mathbf{u}}_{\mathbf{j}}^{n}\right), \quad \overline{\mathbf{v}}_{\mathbf{j}}^{n+\frac{1}{2}} \equiv \frac{1}{2}\left(\overline{\mathbf{v}}_{\mathbf{j}}^{(p)}+\overline{\mathbf{v}}_{\mathbf{j}}^{n}\right), \quad \overline{\mathbf{f}}_{\mathbf{j}}^{n+\frac{1}{2}} \equiv \frac{1}{2}\left(\overline{\mathbf{f}}_{\mathbf{j}}^{(p)}+\overline{\mathbf{f}}_{\mathbf{j}}^{n}\right),
$$

and $\overline{\mathbf{f}}_{\mathbf{j}}^{(p)}$ is defined by $(29)$ with $(\sigma \mathbf{n})^{n}$ replaced by the predicted values $(\sigma \mathbf{n})^{(p)}$.

Stage VI - grid generation: The fluid grid could be corrected and regenerated at this stage of the algorithm. In practice, however, this stage is generally skipped to avoid the cost of an extra grid generation step. Note that the predicted grid is already second-order accurate and there appears to be no noticeable adverse effect on the stability of the algorithm by skipping this stage.

Stage VII - fluid velocity: Corrected values for the fluid velocity are obtained using a second-order accurate Adams-Moulton scheme,

$$
\rho \frac{\mathbf{v}_{\mathbf{i}}^{n+1}-\mathbf{v}_{\mathbf{i}}^{n}}{\Delta t}=\frac{1}{2}\left(\mathbf{F}_{\mathbf{i}}^{(p)}+\mathbf{F}_{\mathbf{i}}^{n}\right), \quad \mathbf{i} \in \Omega_{h},
$$

where $\mathbf{F}_{\mathbf{i}}^{(p)}$ is defined from (30) but with level $n$ values replaced by the predicted values $\mathbf{v}_{\mathbf{i}}^{(p)}$, $\mathbf{w}_{\mathbf{i}}^{(p)}$ and $p_{\mathbf{i}}^{(p)}$. The boundary conditions have the same form as those used in Stage III with $\overline{\mathbf{u}}_{\mathbf{j}}^{(p)}, \overline{\mathbf{v}}_{\mathbf{j}}^{(p)}$ and $\overline{\mathbf{w}}_{\mathbf{j}}^{(p)}$ replaced by $\overline{\mathbf{u}}_{\mathbf{i}}^{n+1}, \overline{\mathbf{v}}_{\mathbf{i}}^{n+1}$ and $\overline{\mathbf{w}}_{\mathbf{j}}^{n+1}$.

Stage VIII - fluid pressure: Corrected values for the pressure are determined by solving the discrete equations in Stage IV with the predicted values replaced by values at $t^{n+1}$.

Stage IX - project interface velocity: Define an interface velocity as a weighted average of the fluid and solid interface velocities, using

$$
\mathbf{v}_{\mathbf{i}}^{I}=\gamma \mathbf{v}_{\mathbf{i}}^{n+1}+(1-\gamma)\left(\overline{\mathbf{v}}_{\mathbf{j}}^{n+1}+\overline{\mathbf{w}}_{\mathbf{j}}^{n+1}\right), \quad \mathbf{i} \in \Gamma_{h}, \quad \mathbf{j} \in \overline{\mathcal{R}}_{h},
$$

where $\gamma$ is defined in (25). Redefine the beam reference-curve velocity as an average of the surface values (adjusted for $\overline{\mathbf{w}}_{\mathbf{k}}^{n+1}$ ),

$$
\overline{\mathbf{v}}_{\mathbf{j}}^{n+1}=\frac{1}{\left|\overline{\mathcal{P}}_{h, \mathbf{j}}\right|} \sum_{\mathbf{k} \in \overline{\mathcal{P}}_{h, \mathbf{j}}}\left(\mathbf{v}_{\mathbf{k}}^{I}-\overline{\mathbf{w}}_{\mathbf{k}}^{n+1}\right) \Delta \theta, \quad \mathbf{j} \in \overline{\mathcal{R}}_{h},
$$

and then redefine the fluid surface velocity to be consistent with the new beam velocity,

$$
\mathbf{v}_{\mathbf{i}}^{n+1}=\overline{\mathbf{v}}_{\mathbf{j}}^{n+1}+\overline{\mathbf{w}}_{\mathbf{j}}^{n+1}, \quad \mathbf{i} \in \Gamma_{h}, \quad \mathbf{j} \in \overline{\mathcal{R}}_{h} .
$$

\section{End corrector.}

We emphasize that the AMP algorithm is stable with no corrector step, although if the predictor step is used alone, then Stage IX should be performed following the predictor to project the interface velocity. We typically use the corrector step, since for the fluid in isolation the scheme has a larger stability region than the predictor step alone, and the stability region includes the imaginary axis so that the scheme can be used for inviscid problems $(\mu=0)$. 


\section{An Euler-Bernoulli beam model}

For the purposes of this paper, we consider a two-dimensional beam governed by a generalized EulerBernoulli (EB) beam model. The model considers the behavior of the displacement, $\eta(s, t)$, of the reference curve of the beam in the direction normal to its initially flat position, where $s$ measures distance along the reference curve. The model assumes small deviations from its initial position (i.e. small slopes) so that displacement in the direction tangent to the reference curve is neglected. The generalized EB model includes damping terms and takes the form

$$
\bar{\rho} \bar{h} \frac{\partial^{2} \eta}{\partial t^{2}}=-\bar{K}_{0} \eta+\frac{\partial}{\partial s}\left(\bar{T} \frac{\partial \eta}{\partial s}\right)-\frac{\partial^{2}}{\partial s^{2}}\left(\bar{E} \bar{I} \frac{\partial^{2} \eta}{\partial s^{2}}\right)-\bar{K}_{1} \frac{\partial \eta}{\partial t}+\bar{T}_{1} \frac{\partial^{2}}{\partial s^{2}}\left(\frac{\partial \eta}{\partial t}\right)+f(s, t), \quad s \in \overline{\mathcal{R}},
$$

where $\bar{\rho}$ is the density of the beam, $\bar{h}=\bar{h}(s)$ is its thickness, $\bar{K}_{0}$ is the linear stiffness coefficient, $\bar{T}$ is the tension coefficient, $\bar{E}$ is Young's modulus, and $\bar{I}$ is the area-moment of inertia of the beam. The term with coefficient $\bar{K}_{1}$ is a linear damping term, while the term with coefficient $\bar{T}_{1}$ damps higher spatial frequencies in $\eta(s, t)$ faster than lower frequencies and thus serves to smooth the beam. We assume that all of the coefficients in (34) are constants, except for the thickness of the beam which may vary with $s$ such as near the rounded end of a cantilevered beam (see Figure 5, for example).

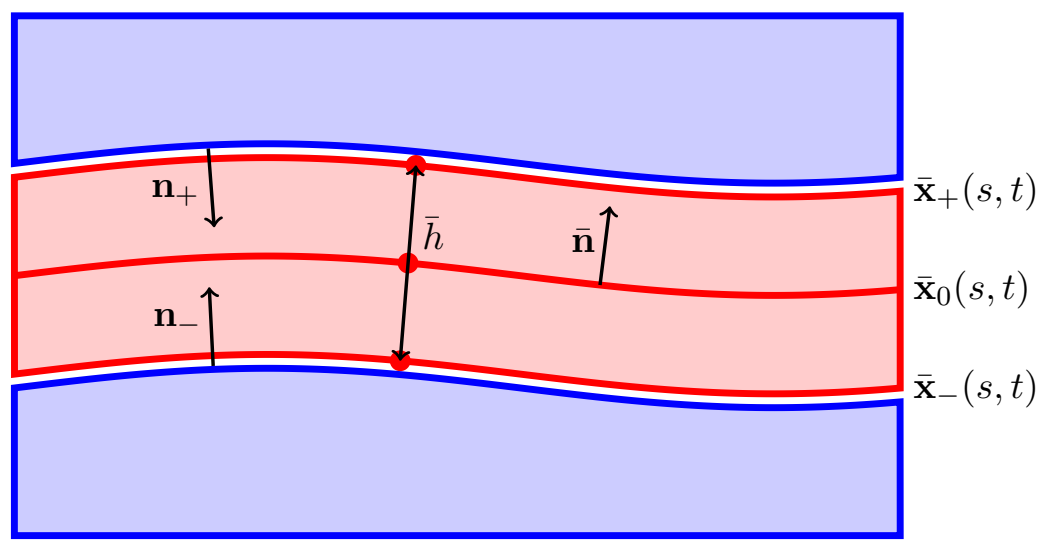

Figure 3: Geometry of an Euler-Bernoulli beam. The beam reference-line is $\overline{\mathbf{x}}_{0}=\overline{\mathbf{x}}_{0}(s, t)$ and the beam normal is $\overline{\mathbf{n}}$. The beam has thickness $\bar{h}$ and meets the fluid on the curves $\overline{\mathbf{x}}_{-}$and $\overline{\mathbf{x}}_{+}$. Outward normals to the fluid domain are $\mathbf{n}_{-}$and $\mathbf{n}_{+}$.

The geometry of an FSI problem involving a section of an EB beam is illustrated in Figure 3. For this configuration, the displacement of the reference curve is given by $\overline{\mathbf{u}}(s, t)=(0, \eta(s, t))$ so that its position is $\overline{\mathbf{x}}_{0}(s, t)=(s, \eta(s, t))$. The unit normal to the reference curve, $\overline{\mathbf{n}}(s, t)$, can be computed in terms of the slope $\partial \eta / \partial s$, and this determines the position of the upper and lower surfaces of the beam as

$$
\overline{\mathbf{x}}_{ \pm}(s, t)=\overline{\mathbf{x}}_{0}(s, t)+\overline{\mathbf{z}}_{ \pm}(s, t), \quad \overline{\mathbf{z}}_{ \pm}(s, t)= \pm \frac{\bar{h}}{2} \overline{\mathbf{n}}(s, t), \quad s \in \overline{\mathcal{R}},
$$

which is a specific case of the formula given previously in (12) with the $b$ subscript dropped for notational convenience. From (35), the velocity of the upper and lower surfaces of the beam are given by

$$
\overline{\mathbf{v}}_{ \pm}(s, t)=\frac{\partial}{\partial t} \overline{\mathbf{x}}_{ \pm}(s, t)=\overline{\mathbf{v}}(s, t)+\overline{\mathbf{w}}_{ \pm}(s, t), \quad \overline{\mathbf{w}}_{ \pm}(s, t)= \pm \frac{\bar{h}}{2} \frac{\partial}{\partial t} \overline{\mathbf{n}}(s, t) .
$$

Finally, a modeling choice is required to determine the force $f(s, t)$ on the reference curve of the beam, and we use

$$
f(s, t)=-\left[\left(\mathbf{n}^{T} \boldsymbol{\sigma} \mathbf{n}\right)_{+}+\left(\mathbf{n}^{T} \boldsymbol{\sigma} \mathbf{n}\right)_{-}\right]
$$


where $\mathbf{n}$ is the unit fluid normal on the beam surface so that $\left(\mathbf{n}^{T} \boldsymbol{\sigma n}\right)_{ \pm}$is the component of the fluid traction in the direction normal to the upper and lower surfaces of the beam. This modeling approximation is valid for small beam deflections, and it is consistent with the use of an EB beam model and the AMP pressure condition in (20).

For a cantilevered beam, the free end of the beam is usually rounded as shown in Figure 5. The flow can be complex near a sharp corner and by rounding the corner a high resolution grid can be generated that resolves the flow in this region. In addition, the force on the beam surface is computed using the beam-surface normal $\mathbf{n}$ and the approximation $\mathbf{n}^{T} \boldsymbol{\sigma} \mathbf{n}$. Near the rounded end of a beam this is a poor approximation since the fluid normal may be orthogonal to the beam reference curve. Consequently, in practice, the fluid forces on the beam tip are ignored in computing force on the beam (in particular, any points on the surface of the beam that extend beyond the length of the beam reference line are ignored). This should be considered a modeling assumption. Similarly, contributions from the end points are also ignored when projecting the interface velocity onto the beam.

\section{Analysis of a model problem for a two-dimensional beam}

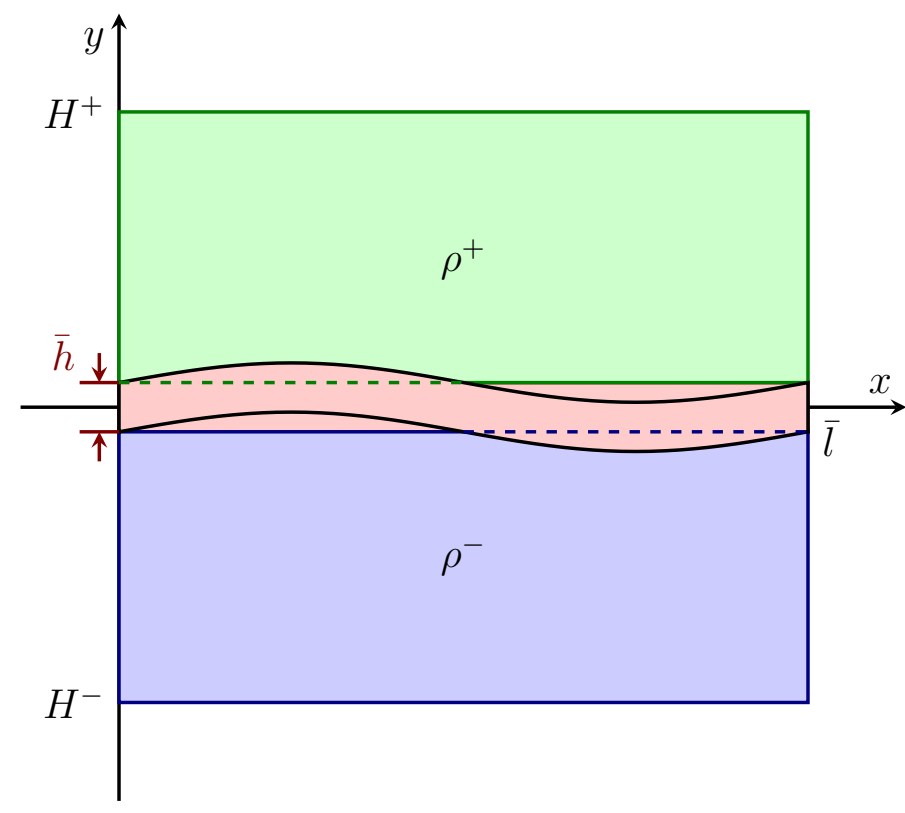

Figure 4: The geometry of the linearized model for a beam with fluid on two sides.

In this section, the stability of the AMP algorithm is investigated for a linearized model problem consisting of an EB beam coupled to inviscid, incompressible fluids with different constant fluid densities in the regions above and below the beam as shown in Figure 4. For comparative purposes, we also consider the stability of a traditional partitioned (TP) scheme for the same model problem. We begin by obtaining the exact solution of the model problem to explicitly identify the contribution of the added mass of the fluid for two different choices of the boundary conditions on the fluid. The stability of the discrete AMP and TP schemes is then investigated using a mode analysis that extends the theory for the case of a beam with fluid on one side only presented in [5]. For both the AMP and TP schemes, the fluid equations are advanced using a backward-Euler time integrator as opposed to the predictor-corrector integrator described in Section 3. While this simplifies the analysis somewhat, the essential results are unaffected. It is found, in agreement with [5], that the AMP scheme is stable for arbitrarily light structures when the usual time-step restriction is satisfied. The TP scheme without sub-time step iteration, on the other hand, becomes unconditionally unstable for light structures. Note that the TP scheme has been analyzed previously by Causin, Gerbeau and Nobile [1], among others, and the results presented here extend those works. 
In the following analysis the two fluids are coupled to an EB beam with constant thickness $\bar{h}$ and without damping terms. The equations describing the evolution of the fluid-structure system are then linearized about flat undeflected beam surfaces at $y=-\bar{h} / 2$ and $\bar{h} / 2$ as shown in Figure 4 . Let $\rho^{+}, \mathbf{v}^{+}(x, y, t)$ and $p^{+}(x, y, t)$, denote the fluid density, velocity and pressure, respectively, in the upper domain, $\Omega_{+}=[0, \bar{l}] \times\left[\bar{h} / 2, H^{+}\right]$, and $\rho^{-}, \mathbf{v}^{-}(x, y, t)$ and $p^{-}(x, y, t)$, the corresponding quantities in the lower domain $\Omega_{-}=[0, \bar{l}] \times\left[H^{-},-\bar{h} / 2\right]$, where $\bar{l}$ is the length of the beam. The governing equations and boundary conditions for this model are

$$
\begin{aligned}
& \text { Fluid: } \begin{cases}\rho^{ \pm} \frac{\partial \mathbf{v}^{ \pm}}{\partial t}+\nabla p^{ \pm}=0, & \mathbf{x} \in \Omega_{ \pm}, \\
\nabla \cdot \mathbf{v}^{ \pm}=0, & \mathbf{x} \in \Omega_{ \pm}, \\
v_{2}^{ \pm}=0 \text { or } p^{ \pm}=0, & x \in(0, \bar{l}), \quad y=H^{ \pm}\end{cases} \\
& \text {Beam: }\left\{\bar{\rho} \bar{h} \frac{\partial^{2} \eta}{\partial t^{2}}=-\bar{K}_{0} \eta+\bar{T} \frac{\partial^{2} \eta}{\partial x^{2}}-\bar{E} \bar{I} \frac{\partial^{4} \eta}{\partial x^{4}}-[p]_{\Gamma}, \quad x \in(0, \bar{l}),\right. \\
& \text { Interface: }\left\{v_{2}^{ \pm}=\frac{\partial \eta}{\partial t}, \quad x \in(0, \bar{l}), \quad y= \pm \frac{\bar{h}}{2}\right. \text {, }
\end{aligned}
$$

where

$$
[p]_{\Gamma} \equiv p^{+}(x, \bar{h} / 2, t)-p^{-}(x,-\bar{h} / 2, t)
$$

denotes the jump of the pressure across the beam, and where we take periodic boundary conditions in the $x$-direction. Initial conditions for $\mathbf{v}^{ \pm}, \eta$ and $\partial \eta / \partial t$ are needed to complete the specification of the problem, but these are not required in the subsequent analysis.

\subsection{Exact solution of the continuous model problem}

It is instructive to first examine the behavior of the exact solution of the FSI problem in (38). Since this model problem is a linear constant-coefficient system of equations with periodic boundary conditions in the $x$-direction, the solution can be expressed in terms of a Fourier series in $x$, e.g.

$$
\mathbf{v}^{ \pm}(x, y, t)=\sum_{k=-\infty}^{\infty} \hat{\mathbf{v}}^{ \pm}(k, y, t) e^{i k_{x} x}, \quad k_{x}=2 \pi k / \bar{l},
$$

and similar expressions for $p^{ \pm}$and $\eta^{ \pm}$. Here, $k_{x}$ identifies the wave number of the Fourier mode. Transforming the equations for the fluid to Fourier space gives

$$
\left.\begin{array}{r}
\rho^{ \pm} \frac{\partial \hat{v}_{1}^{ \pm}}{\partial t}+i k_{x} \hat{p}^{ \pm}=0 \\
\rho^{ \pm} \frac{\partial \hat{v}_{2}^{ \pm}}{\partial t}+\frac{\partial \hat{p}^{ \pm}}{\partial y}=0 \\
i k_{x} \hat{v}_{1}^{ \pm}+\frac{\partial \hat{v}_{2}^{ \pm}}{\partial y}=0
\end{array}\right\}, \quad \begin{aligned}
& y \in\left(H^{-},-\bar{h} / 2\right) \text { for the lower fluid } \\
& y \in\left(\bar{h} / 2, H^{+}\right) \text {for the upper fluid. }
\end{aligned}
$$

The transformed equation for the beam is

$$
\bar{\rho} \bar{h} \frac{\partial^{2} \hat{\eta}}{\partial t^{2}}=-\tilde{L} \hat{\eta}-[\hat{p}]_{\Gamma}
$$

where $\tilde{L}=\bar{K}_{0}+\bar{T} k_{x}^{2}+\bar{E} \bar{I} k_{x}^{4}$, and for notational brevity we have suppressed the functional dependence on the wave number. The transformed equations for the fluid in (39) can be manipulated to derive equations for the pressures of the form

$$
\frac{\partial^{2} \hat{p}^{ \pm}}{\partial y^{2}}-k_{x}^{2} \hat{p}^{ \pm}=0
$$


It is assumed that $k_{x} \neq 0$ in the present analysis while the special case when $k_{x}=0$ is considered later. For the case of the rigid-wall boundary conditions, $\hat{v}_{2}^{ \pm}=0$ at $y=H^{ \pm}$, we use the $y$-momentum equations in (39) to derive the Neumann conditions for the pressures,

$$
\frac{\partial \hat{p}^{ \pm}}{\partial y}\left(H^{ \pm}, t\right)=0
$$

General solutions of the pressure equations in (41) satisfying the homogeneous Neumann boundary conditions in $(42)$ are

$$
\hat{p}^{ \pm}=a^{ \pm} \cosh \left(k_{x}\left(y-H^{ \pm}\right)\right),
$$

where $a^{ \pm}=a^{ \pm}(t)$ are a time-dependent coefficients. Similarly, the kinematic conditions at the interface,

$$
\hat{v}_{2}^{ \pm}( \pm \bar{h} / 2, t)=\frac{\partial \hat{\eta}}{\partial t}
$$

together with the $y$-momentum equations in (39) give the interface conditions

$$
\frac{\partial \hat{p}^{ \pm}}{\partial y}( \pm \bar{h} / 2, t)=-\rho^{ \pm} \frac{\partial^{2} \hat{\eta}}{\partial t^{2}}
$$

The general solutions in (43) and the interface conditions in (44) imply

$$
a^{ \pm}= \pm \frac{\rho^{ \pm}}{k_{x}}\left(\frac{\partial^{2} \hat{\eta}}{\partial t^{2}}\right) \operatorname{csch}\left(k_{x} \mathcal{D}^{ \pm}\right)
$$

where $\mathcal{D}^{ \pm}= \pm H^{ \pm}-\bar{h} / 2$ measure the vertical extent of the upper and lower fluid domains. Using (43) and (45), the applied force appearing in the beam equation (40) takes the form

$$
[\hat{p}]_{\Gamma}=\hat{M}_{a}^{-} \frac{\partial^{2} \hat{\eta}}{\partial t^{2}}+\hat{M}_{a}^{+} \frac{\partial^{2} \hat{\eta}}{\partial t^{2}}
$$

where

$$
\hat{M}_{a}^{ \pm}=\rho^{ \pm} \mathcal{D}^{ \pm}\left[\frac{\operatorname{coth}\left(k_{x} \mathcal{D}^{ \pm}\right)}{k_{x} \mathcal{D}^{ \pm}}\right], \quad \text { (rigid-wall BCs), }
$$

are the added masses from the upper and lower fluids for the case of rigid-wall boundary conditions at $y=H^{ \pm}$. Note that $\rho^{ \pm} \mathcal{D}^{ \pm}$is proportional to the total mass of fluid in the upper and lower domains. For the alternate case of the pressure boundary conditions, $\hat{p}^{ \pm}=0$ at $y=H^{ \pm}$, a similar analysis leads to the added masses given by

$$
\hat{M}_{a}^{ \pm}=\rho^{ \pm} \mathcal{D}^{ \pm}\left[\frac{\tanh \left(k_{x} \mathcal{D}^{ \pm}\right)}{k_{x} \mathcal{D}^{ \pm}}\right], \quad \text { (pressure BCs) }
$$

Finally, using the applied force (46) in the equation of motion for the beam (40) gives

$$
\left(\bar{\rho} \bar{h}+\hat{M}_{a}^{-}+\hat{M}_{a}^{+}\right) \frac{\partial^{2} \hat{\eta}}{\partial t^{2}}=-\tilde{L} \hat{\eta}
$$

which explicitly identifies the contribution of the added mass of the fluid in the beam equation. A solution to (49) satisfying initial conditions for $\hat{\eta}$ and $\partial \hat{\eta} / \partial t$ can be found easily to complete the solution for $\hat{\eta}$. Given $\hat{\eta}$, the pressure is then fully determined from (43) and (45), which in turn determines $\hat{v}_{1}^{ \pm}$and $\hat{v}_{2}^{ \pm}$from (39) together with the initial conditions and boundary conditions. This completes the solution of the model problem in Fourier space.

We now return to the special case when $k_{x}=0$. For rigid-wall boundary conditions, the full solution for $k_{x}=0$ is found to be

$$
\hat{v}_{1}^{ \pm}=\hat{v}_{1}^{ \pm}(y, 0), \quad \hat{v}_{2}^{ \pm}=0, \quad \hat{\eta}=\hat{\eta}(0), \quad \hat{p}^{ \pm}=\hat{p}_{0}^{ \pm}(t),
$$

where $\hat{p}_{0}^{ \pm}(t)$ are spatially uniform fluid pressures whose difference $\Delta \hat{p}_{0}=\hat{p}_{0}^{+}-\hat{p}_{0}^{-}$is a constant related to the beam displacement by

$$
\bar{K}_{0} \hat{\eta}(0)=-\Delta \hat{p}_{0}
$$


Note that the $k_{x}=0$ mode of the beam is stationary, consistent with the fact that the volumes of the lower and upper domains must remain constant for incompressible fluids. The added mass in this case is interpreted to be infinite. This is fully consistent with the observation that the added mass in (47) tends to infinity as $k_{x} \mathcal{D}^{ \pm}$tends to zero. For the case of pressure boundary conditions with $k_{x}=0$, solutions for the fluid pressures become

$$
\hat{p}^{ \pm}=-\rho^{ \pm}\left(\frac{\partial^{2} \hat{\eta}}{\partial t^{2}}\right)\left(y-H^{ \pm}\right)
$$

so that the added masses in (46) are

$$
\hat{M}_{a}^{ \pm}=\rho^{ \pm} \mathcal{D}^{ \pm}
$$

This is in agreement with the limit $k_{x} \mathcal{D}^{ \pm} \rightarrow 0$ for the formula for $\hat{M}_{a}^{ \pm}$in (48).

Returning to the equation for $\hat{\eta}\left(t, k_{x}\right)$ in $(49)$, we note that added mass associated with the fluids on either side of the beam tends to reduce the acceleration of the beam. Since $\hat{M}_{a}^{ \pm} \rightarrow 0$ as $k_{x} \mathcal{D}^{ \pm} \rightarrow \infty$ for either (47) or (48), the effect of the added mass is small for high-frequency components of the solution. For the case of rigid-wall boundary conditions, $\hat{M}_{a}^{ \pm} \rightarrow \infty$ as $\left|k_{x} \mathcal{D}\right|$ becomes small so that the effect of the added mass is large in this limit. This suggests that the rigid-wall case could be difficult numerically if the algorithm is sensitive to the effects of added mass. For the case of the pressure boundary condition, $\hat{M}_{a}^{ \pm}$ becomes constant when $k_{x} \mathcal{D}=0$ and so this case would likely be less difficult numerically than the case of rigid walls.

\subsection{Stability analysis of the AMP and TP schemes}

The stability of a partitioned scheme for an FSI problem depends crucially on the treatment of the interface and the resulting boundary conditions applied to the fluid and structure on either side. AMP interface conditions have been developed and used in the AMP FSI time-stepping algorithm, and the purpose of this section is to analyze the stability of a form of this algorithm applied to the model problem in (38). A starting point for this analysis is the system of equations in (39) and (40) already written in Fourier space. For this system, we consider a discretization in time, but let the dependence on $y$ remain continuous. This approach reveals the stability of the partitioned algorithm, while keeping the algebraic details as simple as possible.

We begin with an analysis of the AMP algorithm, and then compare the results with a traditional partitioned (TP) in the end. For both cases, let

$$
\hat{\mathbf{v}}^{n \pm}(y) \approx \hat{\mathbf{v}}^{ \pm}\left(y, t^{n}\right), \quad \hat{p}^{n \pm}(y) \approx \hat{p}^{ \pm}\left(y, t^{n}\right), \quad \hat{\eta}^{n} \approx \hat{\eta}\left(t^{n}\right),
$$

where $t^{n}=n \Delta t$. Furthermore, let $D_{+t}$ and $D_{-t}$ denote the forward and backward divided different operators in time (e.g. $D_{+t} \hat{\eta}^{n}=\left(\hat{\eta}^{n+1}-\hat{\eta}^{n}\right) / \Delta t$ and $\left.D_{-t} \hat{\eta}^{n}=\left(\hat{\eta}^{n}-\hat{\eta}^{n-1}\right) / \Delta t\right)$.

The following AMP scheme is an abbreviated version of the full scheme described in Section 4: 
AMP scheme:

Stage I: Advance the beam displacement using a leap-frog scheme,

$$
\bar{\rho} \bar{h} D_{+t} D_{-t} \hat{\eta}^{n}=-\tilde{L} \hat{\eta}^{n}-\left[\hat{p}^{n}\right]_{\Gamma} .
$$

Stage II: Advance the fluid velocity and pressure using a backward-Euler scheme applied to the equations in pressure-velocity form,

$$
\left.\begin{array}{c}
\rho^{ \pm} D_{+t} \hat{v}_{1}^{n \pm}+i k_{x} \hat{p}^{(n+1) \pm}=0 \\
\rho^{ \pm} D_{+t} \hat{v}_{2}^{n \pm}+\frac{\partial \hat{p}}{\partial y}^{(n+1) \pm}=0 \\
{\frac{\partial^{2} \hat{p}}{\partial y^{2}}}^{(n+1) \pm}-k_{x}^{2} \hat{p}^{(n+1) \pm}=0
\end{array}\right\}, \quad \begin{aligned}
& y \in\left(H^{-},-\bar{h} / 2\right) \text { for the lower fluid, } \\
& y \in\left(\bar{h} / 2, H^{+}\right) \text {for the upper fluid, }
\end{aligned}
$$

with the AMP Robin interface conditions derived from (20),

$$
-\left[\hat{p}^{n+1}\right]_{\Gamma}+\frac{\bar{\rho} \bar{h}}{\rho^{ \pm}} \frac{\partial \hat{p}^{(n+1) \pm}}{\partial y}=\tilde{L} \hat{\eta}^{n+1}, \quad y= \pm \frac{\bar{h}}{2}
$$

and boundary conditions,

$$
{\frac{\partial \hat{p}^{(n+1) \pm}}{\partial y}}^{(n+}=0, \quad y=H^{ \pm}
$$

A key ingredient of the AMP scheme is the Robin condition in (53) for the fluid pressure. This condition, along with (51), implies

$$
\frac{\partial \hat{p}}{\partial y}^{(n+1) \pm}=-\rho^{ \pm} D_{+t} D_{-t} \hat{\eta}^{n+1}, \quad y= \pm \frac{\bar{h}}{2}
$$

which is a discrete version of the interface condition in (44). We observe that the acceleration term on the right-hand side of (55) is evaluated at the same time level as the pressure gradient term on the left-hand side, and this plays an important role in the stability of the AMP scheme as is noted below. After solving the pressure equation for the fluid subject to the boundary condition in (54) and interface condition in (55), the applied force is found to be

$$
-\left[\hat{p}^{n+1}\right]_{\Gamma}=-\hat{M}_{a}^{-} D_{+t} D_{-t} \hat{\eta}^{n+1}-\hat{M}_{a}^{+} D_{+t} D_{-t} \hat{\eta}^{n+1},
$$

where the added masses, $\hat{M}_{a}^{ \pm}$, are given in (47). Substituting the expression for the applied force (56), with $n+1$ replaced by $n$, into the leap-frog scheme (51) gives the difference equation for $\hat{\eta}^{n}$,

$$
\left(\bar{\rho} \bar{h}+\hat{M}_{a}^{-}+\hat{M}_{a}^{+}\right) D_{+t} D_{-t} \hat{\eta}^{n}=-\tilde{L} \hat{\eta}^{n}
$$

which is analogous to the evolution equation for $\hat{\eta}(t)$ in (49). A solution of the form $\hat{\eta}^{n}=A^{n} \hat{\eta}^{0}$ is sought which leads to the quadratic equation

$$
A^{2}-2\left(1-\frac{\Delta t^{2} \tilde{L}}{2\left(\bar{\rho} \bar{h}+\hat{M}_{a}^{-}+\hat{M}_{a}^{+}\right)}\right) A+1=0,
$$

for the amplitude $A$. Stability requires $|A| \leq 1$ and that the roots be simple, if they have modulus one, which leads to the following theorem: 
Theorem 1. The AMP scheme applied to the model problem in (38) is stable if and only if

$$
\Delta t<2 \sqrt{\frac{\bar{\rho} \bar{h}+\hat{M}_{a}^{-}+\hat{M}_{a}^{+}}{\tilde{L}}},
$$

where $\hat{M}_{a}^{ \pm}$is given by (47) for case of the rigid-wall BCs and by (48) for the case of pressure BCs. Moreover, if (59) holds, then the roots of (58) satisfy $|A|=1$ and the AMP scheme for the model problem is nondissipative.

We note that if a stable time step is chosen for the leap-frog scheme in (51) with no external forcing due to the fluid, namely $\Delta t<2 \sqrt{\bar{\rho} \bar{h} / \tilde{L}}$, then this value of $\Delta t$ would be stable for the AMP scheme as well since $\hat{M}_{a}^{ \pm} \geq 0$. We also note that a velocity projection has been omitted in the prototype AMP scheme considered in the stability analysis, and thus the fluid and structure velocities at the interface would only match to first-order accuracy. An additional projection step to match the velocities on the interface exactly could be included, but this is not essential for the present analysis.

Having considered the stability of the AMP scheme, we now turn our attention to a TP scheme defined as follows:

\section{Traditional partitioned (TP) scheme:}

Stage I: Advance the beam displacement using (51).

Stage II: Advance the fluid velocity and pressure using (52) with interface condition,

$$
\frac{\partial \hat{p}}{\partial y}^{(n+1)^{ \pm}}=-\rho^{ \pm} D_{+t} D_{-t} \hat{\eta}^{n}, \quad y= \pm \frac{\bar{h}}{2},
$$

and the boundary condition in (54).

The only difference between the AMP scheme and the TP scheme is the coupling at the interface. For the TP and AMP schemes, the stress from the fluid at time level $t^{n}$ provides a forcing to the leap-frog scheme in (51) to advance the displacement of the beam to time level $t^{n+1}$. The velocity and pressure in the fluid are then advanced to time level $t^{n+1}$ using the discrete equations in (52), the boundary condition in (54) and a coupling condition at the interface. The AMP scheme uses the coupling condition in (53), whereas the TP scheme uses (60). The latter is derived from the time derivative of the kinematic interface condition matching the vertical velocity of the beam to that of the fluid on interface. We note, however, that a similar interface condition was derived from the leap-frog scheme in (51) and the AMP interface coupling condition in (53), which appears in (55). The key difference is that the time level for the vertical acceleration of the beam in (60) lags that of fluid in contrast to (55), and this is the essential issue leading to the instability of the TP scheme for light structures as the subsequent analysis shows.

Following the steps used in the analysis of the AMP scheme, the pressure equation in (52) is solved with interface conditions in (60) and boundary conditions in (54) to give the applied force

$$
-\left[\hat{p}^{n+1}\right]_{\Gamma}=-\hat{M}_{a}^{-} D_{+t} D_{-t} \hat{\eta}^{n}-\hat{M}_{a}^{+} D_{+t} D_{-t} \hat{\eta}^{n} .
$$

Using the beam equation in (51) together with (61) to eliminate the applied force yields a difference equation for $\hat{\eta}^{n}$ of the form

$$
\bar{\rho} \bar{h} D_{+t} D_{-t} \hat{\eta}^{n}+\left(\hat{M}_{a}^{-}+\hat{M}_{a}^{+}\right) D_{+t} D_{-t} \hat{\eta}^{n-1}=-\tilde{L} \hat{\eta}^{n} .
$$

Again we observe a time lag in the added-mass terms in (62) as compared to the previous evolution equation for $\hat{\eta}^{n}$ in (57) for the AMP scheme. This time-lag is well known and is the primary source of the added-mass instability for the TP scheme. Using $\hat{\eta}^{n}=A^{n} \hat{\eta}^{0}$ in (62) leads to the cubic equation for $A$,

$$
\bar{\rho} \bar{h} A(A-1)^{2}+\Delta t^{2} \tilde{L} A^{2}+\left(\hat{M}_{a}^{-}+\hat{M}_{a}^{+}\right)(A-1)^{2}=0 .
$$


The TP scheme is weakly stable if all roots of (63) satisfy $|A| \leq 1$, and with no repeated roots of modulus one. Using the theory of von Neumann polynomials $[26,27]$ leads to the following theorem.

Theorem 2. For $\bar{\rho} \bar{h}>0$ and $\tilde{L}>0$, the traditional partitioned scheme applied to the model problem in (38) is weakly stable if and only if

$$
\hat{M}_{a}^{-}+\hat{M}_{a}^{+}<\bar{\rho} \bar{h}
$$

and

$$
\Delta t<2 \sqrt{\frac{\bar{\rho} \bar{h}-\hat{M}_{a}^{-}-\hat{M}_{a}^{+}}{\tilde{L}}},
$$

where $\hat{M}_{a}^{ \pm}$is given by (47) for case of the rigid-wall BCs and by (48) for the case of pressure BCs.

The condition in (64) implies that the TP scheme is unconditionally unstable if the contribution from the added mass is too large, i.e. if $\hat{M}_{a}^{-}+\hat{M}_{a}^{+}>\bar{\rho} \bar{h}$, regardless of the choice for the time-step, $\Delta t$. In general, the results of the analysis suggest that TP-type schemes would suffer from stability issues when $\hat{M}_{a}^{ \pm} / \bar{\rho} \bar{h}$ is large. We note from (47) or (48) that $\hat{M}_{a}^{ \pm} / \bar{\rho} \bar{h}$ is large if the mass of the fluid domain is large compared to that of the structure, i.e. if $\rho^{ \pm} \mathcal{D}^{ \pm} / \bar{\rho} h$ is large. However, the ratio is also large for the case of rigid-wall boundary conditions if $\left|k_{x} \mathcal{D}^{ \pm}\right|$is small in view of the bracketed term in (47). This latter condition can be re-interpreted as $\mathcal{D}^{ \pm} / \bar{l}$ is small, i.e. the fluid domain is long and slender, since $k_{x}=2 \pi k / \bar{l}$. These observations are in agreement with the results of Causin et al. [1], who studied a simple beam with fluid on one side. The observations are also in agreement with the numerical results presented here and in [5]. If the condition $\hat{M}_{a}^{-}+\hat{M}_{a}^{+}<\bar{\rho} \bar{h}$ is satisfied, then (65) indicates how the time-step must be reduced as the total added-mass increases.

\subsection{Pressure regularization for rigid-wall boundary conditions}

Note that for both the TP and AMP schemes the limit $k_{x}=0$ with rigid-wall boundary conditions must be treated with some care since the continuous fluid pressure is only determined up to an arbitrary additive constant. In the AMP scheme $p^{+}$and $p^{-}$are coupled so that only the difference in pressure is arbitrary, whereas both pressures are arbitrary for the TP scheme. In a numerical implementation these singularities need to be removed since, due to round-off or truncation errors, the singular systems may have no solution. The specific choice of regularization can affect the overall stability of the approach. For example, one common regularization would follow the approach in [25], which for $k_{x}=0$ would solve the augmented pressure equations

$$
\frac{\partial^{2} \hat{p}^{(n+1) \pm}}{\partial y^{2}}+\alpha^{(n+1) \pm}=0
$$

with the constraints

$$
\int_{H^{-}}^{-\frac{\bar{h}}{2}} \hat{p}^{(n+1)-} d y=\int_{\frac{\bar{h}}{2}}^{H^{+}} \hat{p}^{(n+1)+} d y=0 .
$$

Here $\alpha^{(n+1) \pm}$ are two additional unknowns which serve to regularize the solution and ensure that the augmented system has a unique solution. The effect of regularization can be significant for the $k_{x}=0$ mode. Using this regularization the traditional partitioned scheme is found to be stable for the $k_{x}=0$ mode under the mollified time-step restriction

$$
\Delta t<2 \sqrt{\frac{\bar{\rho} \bar{h}-\frac{1}{3} \rho^{-} \mathcal{D}^{-}-\frac{1}{3} \rho^{+} \mathcal{D}^{+}}{\tilde{L}}} .
$$

This is significant difference from (65) which required $\Delta t \rightarrow 0$ when $k_{x} \rightarrow 0$. This less severe time-step restriction is a result of the pressure regularization and applies only to the $k_{x}=0$ mode. All other small $k_{x}$ modes in the system must satisfy (65). An analogous regularization for the AMP scheme involves the pressure equations

$$
\frac{\partial^{2} \hat{p}^{(n+1) \pm}}{\partial y^{2}}+\alpha^{(n+1)}=0
$$


and the single constraint

$$
\int_{H^{-}}^{-\frac{\bar{h}}{2}} \hat{p}^{(n+1)-} d y+\int_{\frac{\bar{h}}{2}}^{H^{+}} \hat{p}^{(n+1)+} d y=0,
$$

which determines the one unknown $\alpha^{(n+1)}$. In this case it can be shown that the AMP scheme is stable with no time-step restriction for $k_{x}=0$, and the solution is found to be

$$
\hat{v}_{1}^{(n+1) \pm}=\hat{v}_{1}^{0 \pm}, \quad \hat{v}_{2}^{(n+1) \pm}=0, \quad \hat{\eta}^{n+1}=\hat{\eta}^{0}, \quad \hat{p}^{(n+1) \pm}=\mp \bar{K}_{0} \hat{\eta}^{0} \frac{\mathcal{D}^{\mp}}{\mathcal{D}^{+}+\mathcal{D}^{-}} .
$$

Note that, by adding one pressure regularization, the AMP scheme is able to determine the solution uniquely, and the solution is compatible with the exact solution for $k_{x}=0$ given in the continuous case by (50).

\section{Numerical approach using deforming composite grids (DCG)}

Our numerical approach for the solution of the equations governing an FSI initial-boundary-value problem is based on the use of deforming composite grids (DCG). This FSI-DCG approach was first described in [6] for the case of an inviscid compressible flow coupled to a linearly elastic solid, and later in [7] for the case of compressible flow coupled to nonlinear hyperelastic solids. Here, we extend the approach to FSI problems involving an incompressible flow coupled to deforming beams.

\subsection{Deforming composite grids and the fluid domain solver}

Deforming composite grids (DCGs) are used to discretize the evolving fluid domains in physical space. An overlapping grid, $\mathcal{G}$, consists of a set of structured component grids, $\left\{G_{g}\right\}, g=1, \ldots, \mathcal{N}$, that cover each fluid domain, $\Omega_{k}(t)$, and overlap where the component grids meet. Typically, boundary-fitted curvilinear grids are used near the boundaries while one or more background Cartesian grids are used to handle the bulk of the fluid domain. Each component grid is a logically rectangular, curvilinear grid in $n_{d}$ space dimensions, and is defined by a smooth mapping from parameter space $\mathbf{r}$ (the unit square or cube) to physical space $\mathbf{x}$,

$$
\mathbf{x}=\mathbf{g}(\mathbf{r}, t), \quad \mathbf{r} \in[0,1]^{n_{d}}, \quad \mathbf{x} \in \mathbb{R}^{n_{d}} .
$$

Typically, the background grids are static, while the boundary-fitted grids evolve in time to match the motion of the boundary.
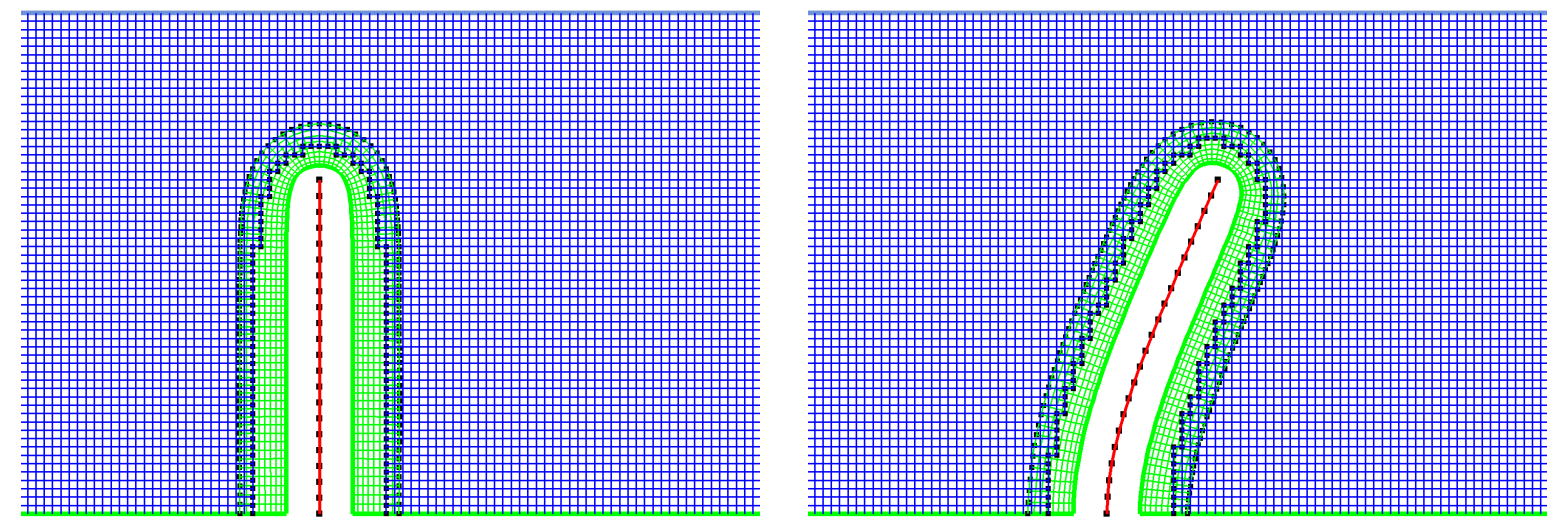

Figure 5: Composite grids for a beam in a channel at times $t=0.0$, and 1.0. The green fluid grid deforms over time to match the evolving beam (shown in white) and overlaps with the background Cartesian fluid grid (shown in blue). The reference curve of the beam is shown in red and the thickness of the beam describes the position of the surface of the beam.

In the FSI-DCG approach, component grids next to a fluid-structure interface deform over time to match the beam motion. This is illustrated in Figure 5 for the case of a beam in a fluid channel. A green fluid grid 
is fitted to the boundary of the beam, which is defined in terms of displacement of the reference curve of the beam (shown in red) and the thickness of the beam (c.f. (35)).

The beam thickness is a function of the position $s \in \overline{\mathcal{R}}$ along the reference curve, and remains fixed as the beam deforms. $\overline{\mathcal{R}}$ is defined from a parameterization of the beam reference curve, e.g. $\overline{\mathcal{R}}=[0, \bar{l}]$ where $\bar{l}$ is the length of the undeformed beam. After each time step, the points on the fluid interface corresponding to the surface of the beam are recomputed according to the current displacement of the beam and the normal to the reference curve using (35), and a hyperbolic grid generator [28] is used to regenerate the local interface grids. After all deforming component grids have been regenerated, the Ogen grid generator [29] is called to regenerate the overlapping grid connectivity (e.g., cut holes, determine interpolation points) between the evolving interface-fitted fluid grids and the fixed background fluid grids, typically Cartesian grids, shown in blue in Figure 5.

The incompressible Navier-Stokes equations are solved in velocity-pressure form on overlapping grids using a second-order accurate fractional-step algorithm [5, 23, 25] similar to that described in Section 4. The treatment of moving overlapping grids, including the assignment of exposed points, follows the approach discussed in [30] for compressible flow. The governing equations for the fluid on a given component grid are solved in a coordinate frame moving with the grid. Consider the momentum equations in (1) for the fluid in an Eulerian frame,

$$
\frac{\partial v_{i}}{\partial t}+v_{j} \frac{\partial v_{i}}{\partial x_{j}}=\frac{1}{\rho} \frac{\partial \sigma_{j i}}{\partial x_{j}}, \quad i=1, \ldots, n_{d},
$$

with summation convention. Under a general moving coordinate transformation, $\mathbf{x}=\mathbf{g}(\mathbf{r}, t)$, the equations in (66) are transformed using the chain rule to become

$$
\frac{\partial v_{i}}{\partial t}+\left(v_{j}-w_{j}\right) \frac{\partial r_{k}}{\partial x_{j}} \frac{\partial v_{i}}{\partial r_{k}}=\frac{1}{\rho} \frac{\partial r_{k}}{\partial x_{j}} \frac{\partial \sigma_{j i}}{\partial r_{k}}, \quad i=1, \ldots, n_{d},
$$

where the component of velocity $v_{i}$ and stress $\sigma_{j i}$ are now considered to be functions of $\mathbf{r}$ and $t$, and where $w_{j}=\partial g_{j} / \partial t$ are the components of the grid velocity, $\mathbf{w}$. The pressure equation in (4) is transformed to $(\mathbf{r}, t)$ in a similar way.

\subsection{Structural domain solvers and the AMP condition}

Two approaches are implemented to advance the governing equations for the beam. The first is a second-order accurate predictor-corrector scheme described previously in Section 4, which also uses standard second-order accurate finite differences to approximate the spatial derivatives in (34) assuming an EulerBernoulli beam. The basic AMP condition in (19) for this beam model becomes

$$
(\boldsymbol{\sigma} \mathbf{n})_{+}+(\boldsymbol{\sigma n})_{-}+\frac{\bar{\rho} \bar{h}}{\rho} \nabla \cdot \boldsymbol{\sigma}\left(\overline{\mathbf{x}}_{ \pm}(s, t), t\right)=\overline{\mathbf{L}}\left(\overline{\mathbf{u}}^{(p)}, \overline{\mathbf{v}}^{(p)}\right)+\bar{\rho} \bar{h} \frac{\partial}{\partial t} \overline{\mathbf{w}}_{ \pm}^{(p)}(s, t), \quad s \in \overline{\mathcal{R}},
$$

where the predicted quantities on the right-hand side of (68) involving the displacement and velocity of the beam can be computed in terms of the discrete values for $\eta, \partial \eta / \partial t$, and $\partial^{2} \eta / \partial t^{2}$, and their spatial derivatives, using standard finite differences. The normal and tangential components of (68) then become boundary conditions for the fractional-step fluid solver.

The second approach implemented to advance the equations in (34) for an Euler-Bernoulli beam uses a standard finite-element approximation in space and the Newmark-beta time-stepping scheme (given below). The spatial representation of the beam displacement in the finite-element approximation is taken as

$$
\eta_{h}(s, t)=\sum_{j=1}^{N_{n}}\left\{\eta_{j}(t) \phi_{j}(s)+\eta_{j}^{\prime}(t) \psi_{j}(s)\right\},
$$

where the degrees of freedom are the nodal displacement $\eta_{j}(t)$ and slope $\eta_{j}^{\prime}(t)$, and where $\phi_{j}(s)$ and $\psi_{j}(s)$ are cubic Hermite polynomials with support on the interval $\left[s_{j-1}, s_{j+1}\right]$ and satisfying

$$
\begin{array}{ll}
\phi_{i}\left(s_{j}\right)=\delta_{i j}, & \phi_{i}^{\prime}\left(s_{j}\right)=0, \\
\psi_{i}\left(s_{j}\right)=0, & \psi_{i}^{\prime}\left(s_{j}\right)=\delta_{i j},
\end{array}
$$


where $\delta_{i j}$ is the Kronecker delta. Following the usual Galerkin approximation in space, the beam equation becomes

$$
M_{h} \frac{d^{2} \boldsymbol{\eta}_{h}}{d t^{2}}=-K_{h} \boldsymbol{\eta}_{h}-B_{h} \frac{d \boldsymbol{\eta}_{h}}{d t}+\mathbf{f}_{h},
$$

where $M_{h}$ is a mass matrix, $K_{h}$ is a stiffness matrix, and $B_{h}$ is a damping matrix. The vector $\boldsymbol{\eta}_{h}$ contains the nodal displacements and slopes, $\eta_{j}(t)$ and $\eta_{j}^{\prime}(t), j=1, \ldots, N_{n}$, and the components of the forcing vector $\mathbf{f}_{h}$ are given by integrals of $f(s, t)$ in (34) with the Hermite basis functions ${ }^{6}$.

The ODEs in (70) are advanced in time using a second-order accurate Newmark-beta scheme in predictorcorrector form. Let $\boldsymbol{\eta}_{h}^{n} \approx \boldsymbol{\eta}_{h}\left(t^{n}\right), \dot{\boldsymbol{\eta}}_{h}^{n} \approx \frac{d \boldsymbol{\eta}_{h}}{d t}\left(t^{n}\right), \mathbf{a}_{h}^{n} \approx \frac{d^{2} \boldsymbol{\eta}_{h}}{d t^{2}}\left(t^{n}\right)$, and $\mathbf{f}_{h}^{n} \approx \mathbf{f}_{h}\left(t^{n}\right)$. The Newmark-beta scheme is based on a Taylor series in time and is given by

$$
\begin{aligned}
& \boldsymbol{\eta}_{h}^{n+1}=\boldsymbol{\eta}_{h}^{n}+\Delta t \dot{\boldsymbol{\eta}}_{h}^{n}+\frac{\Delta t^{2}}{2}\left[(1-2 \beta) \mathbf{a}_{h}^{n}+2 \beta \mathbf{a}_{h}^{n+1}\right], \\
& \dot{\boldsymbol{\eta}}_{h}^{n+1}=\dot{\boldsymbol{\eta}}_{h}^{n}+\Delta t\left[(1-\gamma) \mathbf{a}_{h}^{n}+\gamma \mathbf{a}_{h}^{n+1}\right] \\
& M_{h} \mathbf{a}_{h}^{n+1}=-K_{h} \boldsymbol{\eta}_{h}^{n+1}-B_{h} \dot{\boldsymbol{\eta}}_{h}^{n+1}+\mathbf{f}_{h}^{n+1}
\end{aligned}
$$

We take $\beta=1 / 4$ and $\gamma=1 / 2$ which defines a second-order accurate approximation. During the prediction step, the forcing $\mathbf{f}_{h}^{n+1}$ is not yet known in FSI problems and instead a predicted value is used given by extrapolation in time

$$
\mathbf{f}_{h}^{(p)}=2 \mathbf{f}_{h}^{n}-\mathbf{f}_{h}^{n-1} .
$$

Given predicted values for $\boldsymbol{\eta}_{h}^{(p)}$ and $d \boldsymbol{\eta}_{h}^{(p)} / d t$ from the first stage of the AMP algorithm, pointwise values of the beam operator, $\overline{\mathbf{L}}^{(p)}$, are required to evaluate the right-hand side of the AMP condition in (68). This is generally a straightforward computation in the context of finite difference or finite volume approximations to the beam operator, whereas the calculation may be more difficult within the finite-element approximation. As an example, consider a beam operator of the form

$$
\bar{L}=-\left(\bar{E} \bar{I} \eta_{s s}\right)_{s s}
$$

where the subscripts denote partial derivatives with respect to $s$. It is sufficient to only consider the highest derivative term in (34) for the purposes of the present discussion. Let $\bar{L}$ have the Hermite finite-element representation

$$
\bar{L}_{h}(s, t)=\sum_{j=1}^{N_{n}}\left\{\gamma_{j}(t) \phi_{j}(s)+\gamma_{j}^{\prime}(t) \psi_{j}(s)\right\},
$$

where $\gamma_{j}(t)$ and $\gamma_{j}^{\prime}(t)$ are coefficient functions of time, and $\phi_{j}(s)$ and $\psi_{j}(s)$ are the cubic polynomial basis functions in (69). The $2 N_{n}$ unknowns, $\gamma_{j}(t)$ and $\gamma_{j}^{\prime}(t)$, are determined from the $2 N_{n}$ Galerkin equations

$$
\left(\chi_{j}, \bar{L}_{h}\right)_{\overline{\mathcal{R}}}=\left(\chi_{j},-\left(\bar{E} \bar{I} \eta_{h, s s}\right)_{s s}\right)_{\overline{\mathcal{R}}}, \quad j=1,2, \ldots, N_{n}
$$

where $\chi_{j}=\phi_{j}$ or $\psi_{j}$, and where $(f, g)_{\overline{\mathcal{R}}} \equiv \int_{0}^{\bar{l}} f(s) g(s) d s$. Integration by parts (twice) gives

$$
\left.\left(\chi_{j}, \bar{L}_{h}\right)_{\overline{\mathcal{R}}}=-\left(\chi_{j, s s}, \bar{E} \bar{I} \eta_{h, s s}\right)_{\overline{\mathcal{R}}}+\left\{-\chi_{j}\left(\bar{E} \bar{I} \eta_{h, s s}\right)_{s}+\chi_{j, s} \bar{E} \bar{I} \eta_{h, s s}\right)\right\}\left.\right|_{0} ^{\bar{l}}, \quad j=1,2, \ldots, N_{n} .
$$

The problem now arises in computing second-order accurate approximations to the boundary terms $\left(\bar{E} \bar{I} \eta_{h, s s}\right)_{s}$ and $\bar{E} \bar{I} \eta_{h, s s}$ appearing in (73). The cubic Hermite representation is not of sufficient accuracy for this purpose since, for example, $\eta_{h, s s s}$ is constant over an element and at best first-order accurate.

There are various possible solutions to the difficulty of evaluating the right-hand side of (68) in the context of a finite-element approximation, and we have considered and tested two different approaches that require relatively minor changes. The properties of the two approaches are evaluated in Section 8 where some conclusions are drawn.

\footnotetext{
${ }^{6}$ Appropriate adjustments are made to the matrices and right-hand-side to account for boundary conditions.
} 


\subsubsection{AMP-PBA : Predict Beam Acceleration}

In the first approach, which we refer to as AMP-PBA (Predict Beam Acceleration), the vector form of (34) is used in (68) to eliminate $\overline{\mathbf{L}}^{(p)}$, which gives an AMP condition of the form,

$$
(\boldsymbol{\sigma} \mathbf{n})_{+}+(\boldsymbol{\sigma} \mathbf{n})_{-}+\frac{\bar{\rho} \bar{h}}{\rho} \nabla \cdot \boldsymbol{\sigma}\left(\overline{\mathbf{x}}_{ \pm}(s, t), t\right)=\bar{\rho} \bar{h} \frac{\partial^{2}}{\partial t^{2}} \overline{\mathbf{u}}^{(p)}-\overline{\mathbf{f}}^{(p)}(s, t)+\bar{\rho} \bar{h} \frac{\partial}{\partial t} \overline{\mathbf{w}}_{ \pm}^{(p)}(s, t), \quad s \in \overline{\mathcal{R}} .
$$

The predicted beam acceleration $\partial^{2} \overline{\mathbf{u}}^{(p)} / \partial t^{2}$ in (74) is readily computed from the acceleration term in the weak form (70), while the predicted fluid forcing, $\overline{\mathbf{f}}^{(p)}(s, t)=-(\boldsymbol{\sigma} \mathbf{n})_{+}^{(p)}-(\boldsymbol{\sigma} \mathbf{n})_{-}^{(p)}$, can be obtained from the discrete fluid stress. This gives the following AMP-PBA algorithm:

1. Obtain predicted values for $\overline{\mathbf{f}}^{(p)}=-(\boldsymbol{\sigma} \mathbf{n})_{+}^{(p)}-(\boldsymbol{\sigma} \mathbf{n})_{-}^{(p)}$ using extrapolation in time during the predictor stage or the current guess during the corrector stage (c.f. the algorithm in Section 4).

2. Solve (70) for $d^{2} \boldsymbol{\eta}_{h}^{(p)} / d t^{2}$ using the predicted force $\overline{\mathbf{f}}^{(p)}$, and evaluate pointwise values for $\bar{\rho} \bar{h}\left(\partial^{2} \overline{\mathbf{u}}^{(p)} / \partial t^{2}\right)$ from the Hermite representation for this quantity.

3. Evaluate the right-hand side to (74) and use this when applying the AMP conditions to the fluid.

\subsubsection{AMP-PBF : Predict Beam internal Force}

In the second approach, referred to as AMP-PBF (Predict Beam internal Force), a mixed finiteelement/finite-difference approach is used to compute the beam internal force $\overline{\mathbf{L}}^{(p)}$ directly from equation (73) (extended to include all terms in the full EB model). In particular, using the solution nodal values and derivatives on the boundary and two adjacent points, a fourth-order accurate approximation to $\bar{E} \bar{I} \eta_{s s}$ and a second-order accurate approximation to $\left(\bar{E} \bar{I} \eta_{s s}\right)_{s}$ on the boundary can be derived. This allows the coefficients $\gamma_{j}(t)$ and $\gamma_{j}^{\prime}(t)$ to be determined and then pointwise values of $\overline{\mathbf{L}}^{(p)}$ can be computed from (71). This gives the following AMP-PBF algorithm:

1. Given predicted values for $\eta_{h}^{(p)}$, determine second-order accurate approximations to the boundary terms $\left(\bar{E} \bar{I} \eta_{h, s s}\right)_{s}$ and $\bar{E} \bar{I} \eta_{h, s s}$ in (73) using a finite-difference approximation involving the boundary points and two adjacent points.

2. Compute values for $\overline{\mathbf{L}}^{(p)}$ by solving the Galerkin equations in (73) (extended to include all terms in the full EB model) for $\gamma_{j}$ and $\gamma_{j}^{\prime}$.

3. Evaluate the right-hand side to (68) using (71), and use this when applying the AMP conditions to the fluid.

\subsection{Filtering the projected interface velocity}

The projected interface velocity (24) is optionally smoothed using a few iterations of a fourth-order filter as described in [31]. The beam solution can also be smoothed to prevent high-frequency numerical oscillations, for example by choosing the coefficient $\bar{T}_{1}$ in (34) to be proportional to $\Delta s^{2}$. However, for light beams the interface velocity is determined primarily from the fluid and thus the filter on the interface velocity is needed for light beams to prevent numerical oscillations on the interface. Note that as the beam becomes very light, with beam parameters $\bar{\rho} \bar{h}, \bar{T}, \bar{E} \bar{I}$, etc. going to zero, the AMP interface condition approaches that of a free surface boundary condition for the fluid. Such a free surface condition with no surface tension is susceptible to physical and numerical instabilities, and the fourth-order filter acts to suppress these instabilities and thus serves as a numerical surface tension. This filter does not affect the overall second-order accuracy of the AMP FSI time-stepping scheme.

\subsection{Time step determination}

The global time step, $\Delta t$, for the AMP FSI time-stepping scheme is chosen to be the minimum of the stable time steps for the fluid domain solver and beam solver. The $\Delta t$ for the fluid solver is chosen to be a factor $\lambda_{\mathrm{SF}}$ ("SF" for stability factor) times an estimate of the largest stable time step based on a von Neumann analysis of the linearized Navier-Stokes system. Typically we choose $\lambda_{\mathrm{SF}}=0.9$. In this work, the FEM beam solver is implicit in time and stable for any time step. However, for accuracy reasons it is convenient to choose $\Delta t$ based on a condition relating to explicit time-stepping. Thus, the time-step for the beam is chosen as a factor $\bar{\lambda}_{\mathrm{SF}}$ times an estimate of the largest time step such that an explicit time-stepping procedure for the beam would satisfy a CFL stability condition. Here $\bar{\lambda}_{\mathrm{SF}}$ is chosen in the range 1 to 10 . 


\section{Numerical Results}

We now present the results for a series of simulations chosen to demonstrate the properties of our numerical approach for FSI problems based on the use of the AMP interface conditions. In order to illustrate the basic properties of the approach in a simple setup, we begin with two cases that use a single deforming fluid grid coupled to an Euler-Bernoulli (EB) beam with negligible thickness and finite mass. This simplification eliminates the finite-thickness corrections given in (35) and (36) by setting $\bar{h}=0$. The first test involves a time-dependent problem in which an exact solution is known following the method of manufactured solutions, while the second test considers a steady state FSI problem with known analytical solution. The solutions for both tests are obtained using the beam solver based on finite differences, and they illustrate the accuracy and stability of our numerical approach for a relatively simple geometric configuration. The next set of tests consider finite-thickness EB beams in more complex geometries. Solutions of these FSI problems are obtained using deforming composite grids and the beam solver based on finite elements as discussed in Section 7. These problems include the dynamics of a flexible beam separating two fluid chambers, fluid flow in a channel with flexible walls, and finally the deformation of a flexible cantilevered beam in a cross fluid flow.

\subsection{Verification results for a beam with negligible thickness}

We first consider solutions of two FSI problems involving the interaction of an incompressible fluid and a beam of negligible thickness and finite mass. The geometric configuration of both problems is illustrated in Figure 6. The fluid domain is given by $\Omega(t)=[0,1] \times[0,1+\eta(x, t)]$, where $\eta(x, t)$ is the vertical displacement of the Euler-Bernoulli (EB) beam which forms the upper surface of the fluid domain. The fluid domain is represented by a single transfinite interpolation (TFI) grid which evolves in time according to the computed solution for $\eta$. The TFI mapping is given by

$$
(x, y)=\mathbf{g}(\mathbf{r}, t) \equiv\left(r_{1},\left(1+\eta\left(r_{1}, t\right)\right) r_{2}\right), \quad \mathbf{r} \in[0,1] \times[0,1] .
$$

The equations for the fluid are solved in the mapped domain on the unit square with uniform grid spacings $\Delta r_{1}=\Delta r_{2}$. The equations for the EB beam are solved for $s=r_{1} \in[0,1]$ using the same grid spacing as the fluid grid in the $r_{1}$ direction.

\subsubsection{Manufactured Solutions}

The method of manufactured solutions [32,33] can be used to construct exact solutions of FSI problems by adding forcing functions to the governing equations. The forcing is specified so that a chosen function becomes an exact solution to the forced equations. Here the approach is used to verify the stability and accuracy of the AMP scheme for the nonlinear FSI problem described above consisting of an initially rectangular fluid domain bounded by an EB beam along its top surface.

The parameters for the fluid are chosen to be $\rho=1$ and $\mu=0.05$. The thickness of the beam is taken to be zero while the finite mass of the beam given by $\bar{\rho} \bar{h}$ is varied to consider the cases of light, medium and heavy beams. The remaining parameters of the beam are assumed to be $\bar{K}=1, \bar{T}=\bar{\rho} \bar{h}$ and $\bar{E} \bar{I}=\bar{K}_{1}=\bar{T}_{1}=0$. The exact displacement of the beam is chosen to be

$$
\eta_{e}(x, t)=\frac{a}{\pi f_{t}} \sin \left(f_{x} \pi x\right) \sin \left(f_{t} \pi t\right),
$$

where $a=0.5$ is an amplitude and $f_{x}=f_{t}=2$ are frequencies. (Note that $s=x=r_{1}$ for this problem.) The exact components of velocity and the pressure in the fluid are taken to be

$$
\left.\begin{array}{l}
v_{1, e}(\mathbf{x}, t)=-a \cos \left(f_{x} \pi x\right) \sin \left(f_{x} \pi \tilde{y}\right) \cos \left(f_{t} \pi t\right), \\
v_{2, e}(\mathbf{x}, t)=a \sin \left(f_{x} \pi x\right) \cos \left(f_{x} \pi \tilde{y}\right) \cos \left(f_{t} \pi t\right)-a \frac{\partial \eta_{e}}{\partial x} \cos \left(f_{x} \pi x\right) \sin \left(f_{x} \pi \tilde{y}\right) \cos \left(f_{t} \pi t\right), \\
p_{e}(\mathbf{x}, t)=\cos \left(f_{x} \pi x\right) \cos \left(f_{x} \pi y\right) \cos \left(f_{t} \pi t\right),
\end{array}\right\}
$$

where $\tilde{y}=y-\left(1+\eta_{e}\right)$. The components of velocity have been chosen so that the continuity equation is satisfied in the exact solution, and the vertical velocities of the fluid and the beam match along the interface 
at $y=\eta_{e}$. Forcing functions are added to the equations for the fluid and structure so that the functions in (75) and (76) are exact solutions.

The stability of the AMP scheme for light beams is illustrated in figure 6 for the case $\bar{\rho} \bar{h}=10^{-3}$. The image on the left shows the TFI grid for the fluid domain at $t=0.1$. The beam lies on the top surface of the domain, while the boundary conditions on the other three sides of the domain are taken to be no-slip conditions on the left and right sides $\left(\mathbf{v}=\mathbf{v}_{e}\right)$ and Dirichlet conditions on the bottom $\left(\mathbf{v}=\mathbf{v}_{e}\right.$ and $\left.p=p_{e}\right)$. The plot in the center of the figure shows the numerical solution for the vertical component of the fluid velocity, $v_{2}$, while the plot on the right shows the difference between the computed solution for $v_{2}$ and the exact solution given by $v_{2, e}$. Here we observe that the error in $v_{2}$ is well behaved in that the magnitude is small and it is smooth throughout the domain including the boundaries. The behavior of the error in the other components of the solution are similar.
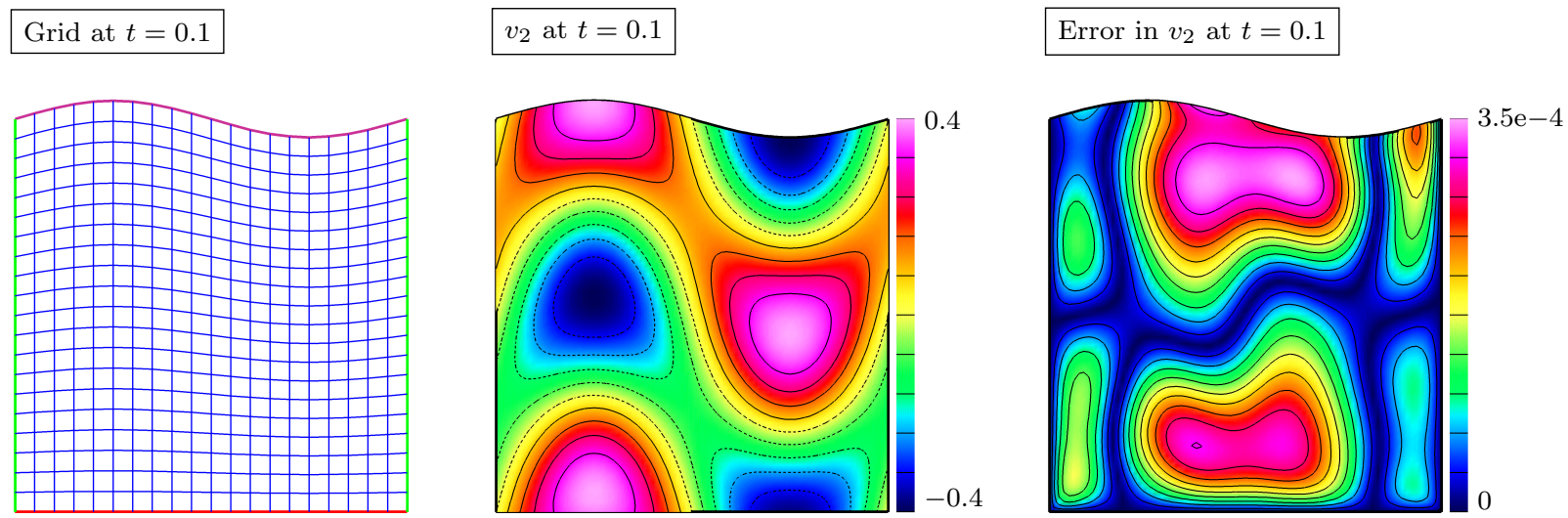

Figure 6: Manufactured solutions: computational results at $t=0.1$ for a light beam $\left(\bar{\rho} \bar{h}=10^{-3}\right)$ using a grid spacing of $h=1 / 80$. Left: deformed grid (coarsened version). Middle: solution component $v_{2}$. Right: error in $v_{2}$.

A convergence study is shown in Table 7 for light $\left(\bar{\rho} \bar{h}=10^{-3}\right)$, medium $(\bar{\rho} \bar{h}=1)$ and heavy $\left(\bar{\rho} \bar{h}=10^{3}\right)$ beams using grid spacings $\Delta r_{1, j}=\Delta r_{2, j}=h_{j}=1 /(10 j), j=1,2, \ldots$ The maximum-norm errors, $E_{j}^{(q)}$ for solution component $q$ and for grid resolutions $j=1,2,4$ and 8 , are computed for each value of $\bar{\rho} \bar{h}$. The convergence rate is estimated by a least squares fit to the logarithm of the error over the grid resolutions. The results in the table for the components of the fluid velocity $\left(v_{1}, v_{2}\right)$ and pressure $p$, and for the beam displacement $\eta$ and velocity $\eta_{t}$ show the expected second-order accuracy for all three choices of the beam mass, and in particular for the case of a light beam when added-mass effects are strong.

\subsubsection{Steady state beam bounding a pressurized fluid chamber}

To illustrate the effectiveness of the AMP coupling scheme in comparison to the traditional FSI coupling, we now discuss a problem where the beam deforms as a result of a uniform fluid pressure applied at the bottom of the fluid domain. For this problem, an exact solution for the long-time steady state deflection of the beam can be found. The steady state can be computed numerically by integrating the time-dependent equations from an initially flat beam. It is found that for light beams the traditional scheme is unstable, in agreement with the previous stability analysis, while the AMP scheme remains stable and the steady state solution is computed accurately.

For this FSI problem, the material parameters for the fluid are chosen as $\rho=1$ and $\mu=0.2$, while those for the beam are chosen as $\bar{K}=0, \bar{T}=5, \bar{E} \bar{I}=0, \bar{K}_{1}=\bar{T}_{1}=0$ and a finite value for $\bar{\rho} \bar{h}$. The boundary conditions on the left and right sides of the fluid domain are given as a no-slip walls $(\mathbf{v}=0)$, while a given pressure $\left(p=P_{a}=5\right)$ and zero tangential velocity $\left(v_{1}=0\right)$ are assigned on the bottom of the fluid domain. One boundary condition is required at each end of the beam and these are taken as $\eta=0$ at each end. (The highest spatial derivative in the beam model is two for the chosen parameters.) The initial conditions are 


\begin{tabular}{|c|c|c|c|c|c|c|c|c|c|c|}
\hline \multicolumn{10}{|c|}{ light beam, $\bar{\rho} \bar{h}=10^{-3}$} \\
\hline$h_{j}$ & $E_{j}^{(p)}$ & $r$ & $E_{j}^{\left(v_{1}\right)}$ & $r$ & $E_{j}^{\left(v_{2}\right)}$ & $r$ & $E_{j}^{(\eta)}$ & $r$ & $E_{j}^{\left(\eta_{t}\right)}$ & $r$ \\
\hline $1 / 10$ & $6.55 \mathrm{e}-02$ & & $2.64 \mathrm{e}-02$ & & $2.23 \mathrm{e}-02$ & & $9.06 \mathrm{e}-04$ & & $2.19 \mathrm{e}-02$ & \\
\hline $1 / 20$ & $1.70 \mathrm{e}-02$ & 3.84 & $7.26 \mathrm{e}-03$ & 3.64 & $5.41 \mathrm{e}-03$ & 4.12 & $2.23 \mathrm{e}-04$ & 4.07 & $5.15 \mathrm{e}-03$ & 4.25 \\
\hline $1 / 40$ & $4.45 \mathrm{e}-03$ & 3.83 & $1.86 \mathrm{e}-03$ & 3.91 & $1.39 \mathrm{e}-03$ & 3.90 & $6.03 \mathrm{e}-05$ & 3.69 & $1.31 \mathrm{e}-03$ & 3.94 \\
\hline $1 / 80$ & $1.12 \mathrm{e}-03$ & 3.98 & $4.70 \mathrm{e}-04$ & 3.95 & $3.55 \mathrm{e}-04$ & 3.91 & $1.54 \mathrm{e}-05$ & 3.90 & $3.29 \mathrm{e}-04$ & 3.97 \\
\hline rate & 1.96 & & 1.94 & & 1.99 & & 1.95 & & 2.01 & \\
\hline
\end{tabular}

\begin{tabular}{|c|c|c|c|c|c|c|c|c|c|c|}
\hline \multicolumn{10}{|c|}{ medium beam, $\bar{\rho} \bar{h}=1$} \\
\hline$h_{j}$ & $E_{j}^{(p)}$ & $r$ & $E_{j}^{\left(v_{1}\right)}$ & $r$ & $E_{j}^{\left(v_{2}\right)}$ & $r$ & $E_{j}^{(\eta)}$ & $r$ & $E_{j}^{\left(\eta_{t}\right)}$ & $r$ \\
\hline $1 / 10$ & $1.18 \mathrm{e}-01$ & & $2.07 \mathrm{e}-02$ & & $2.40 \mathrm{e}-02$ & & $3.56 \mathrm{e}-04$ & & $9.09 \mathrm{e}-03$ & \\
\hline $1 / 20$ & $2.53 \mathrm{e}-02$ & 4.69 & $5.86 \mathrm{e}-03$ & 3.53 & $5.80 \mathrm{e}-03$ & 4.14 & $7.70 \mathrm{e}-05$ & 4.63 & $1.89 \mathrm{e}-03$ & 4.80 \\
\hline $1 / 40$ & $6.49 \mathrm{e}-03$ & 3.90 & $1.54 \mathrm{e}-03$ & 3.80 & $1.52 \mathrm{e}-03$ & 3.82 & $2.02 \mathrm{e}-05$ & 3.81 & $4.61 \mathrm{e}-04$ & 4.11 \\
\hline $1 / 80$ & $1.67 \mathrm{e}-03$ & 3.89 & $3.95 \mathrm{e}-04$ & 3.90 & $3.87 \mathrm{e}-04$ & 3.92 & $5.10 \mathrm{e}-06$ & 3.96 & $1.15 \mathrm{e}-04$ & 4.02 \\
\hline rate & 2.04 & & 1.91 & & 1.98 & & 2.03 & & 2.10 & \\
\hline
\end{tabular}

\begin{tabular}{|c|c|c|c|c|c|c|c|c|c|c|}
\hline \multicolumn{10}{|c|}{ heavy beam, $\bar{\rho} \bar{h}=10^{3}$} \\
\hline$h_{j}$ & $E_{j}^{(p)}$ & $r$ & $E_{j}^{\left(v_{1}\right)}$ & $r$ & $E_{j}^{\left(v_{2}\right)}$ & $r$ & $E_{j}^{(\eta)}$ & $r$ & $E_{j}^{\left(\eta_{t}\right)}$ & $r$ \\
\hline $1 / 10$ & $1.73 \mathrm{e}-01$ & & $2.06 \mathrm{e}-02$ & & $2.80 \mathrm{e}-02$ & & $8.45 \mathrm{e}-05$ & & $2.91 \mathrm{e}-03$ & \\
\hline $1 / 20$ & $3.33 \mathrm{e}-02$ & 5.20 & $5.63 \mathrm{e}-03$ & 3.66 & $6.68 \mathrm{e}-03$ & 4.20 & $2.25 \mathrm{e}-05$ & 3.76 & $7.72 \mathrm{e}-04$ & 3.77 \\
\hline $1 / 40$ & $8.83 \mathrm{e}-03$ & 3.77 & $1.51 \mathrm{e}-03$ & 3.74 & $1.77 \mathrm{e}-03$ & 3.77 & $6.37 \mathrm{e}-06$ & 3.53 & $1.91 \mathrm{e}-04$ & 4.05 \\
\hline $1 / 80$ & $2.31 \mathrm{e}-03$ & 3.82 & $3.90 \mathrm{e}-04$ & 3.86 & $4.54 \mathrm{e}-04$ & 3.90 & $1.63 \mathrm{e}-06$ & 3.92 & $4.75 \mathrm{e}-05$ & 4.01 \\
\hline rate & 2.06 & & 1.91 & & 1.98 & & 1.89 & & 1.98 & \\
\hline
\end{tabular}

Figure 7: Maximum-norm errors and estimated convergence rates using manufactured solutions. The column labeled "r" provides the ratio of the errors at the current grid spacing to that on the next coarser grid.
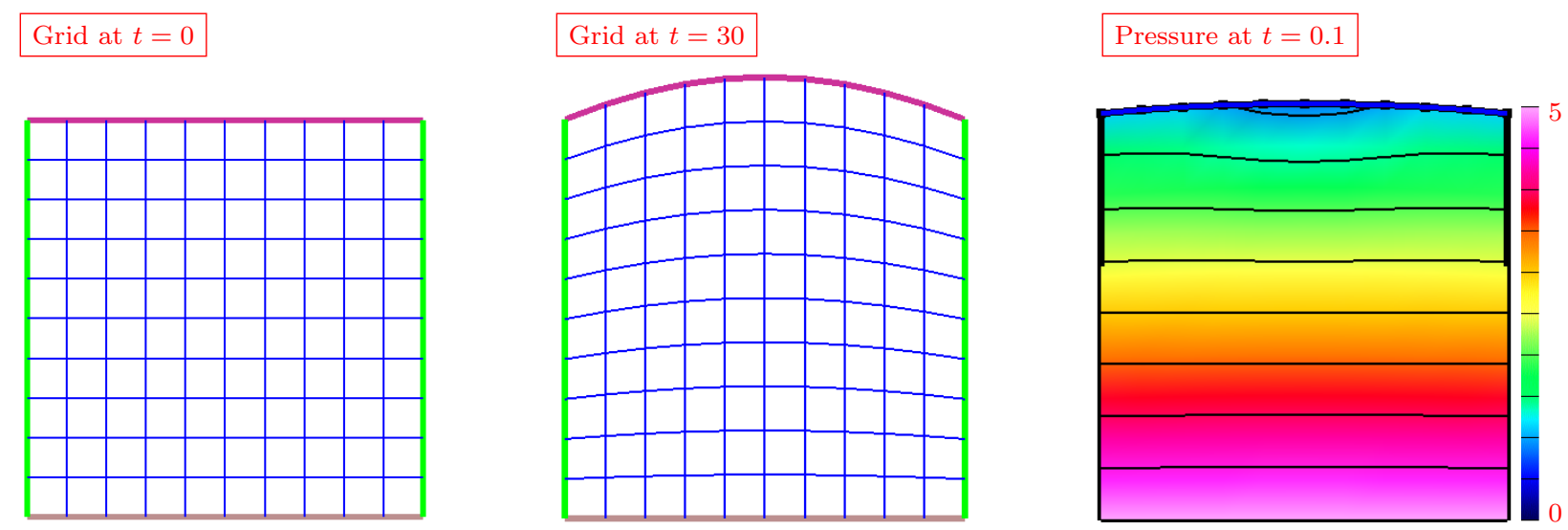

Figure 8: Computational grids and pressure contour plots for a light beam $\left(\bar{\rho} \bar{h}=10^{-3}\right)$ bounding a pressurized fluid chamber using a grid spacing of $h=1 / 10$. Left: initial grid. Middle: steady state grid. Right: pressure contour.

given as a flat stationary beam $(\eta=\dot{\eta}=0)$ and a fluid at rest. The exact steady-state solution is

$$
\mathbf{v}=0, \quad p=P_{a}, \quad \eta=\frac{P_{a} x(1-x)}{2 \bar{T}}, \quad x \in[0,1] .
$$

As before, $\bar{\rho} \bar{h}$ is varied to illustrate the stability of the time-integration of the equations using the AMP coupling scheme for a range of masses of the beam, and also to highlight the instability of the traditional FSI coupling scheme for light beams.

The computational grids for the case of a light beam $\left(\bar{\rho} \bar{h}=10^{-3}\right)$ at initial and steady states are shown in the left and middle images of Figure 8; a contour plot of the pressure at an early time $(t=0.1)$ is also 
included in the right image of Figure 8 to show the evolution of the system. Figure 9 shows the behavior of the displacement and velocity of the beam at its center, $x=1 / 2$, as a function of time for the cases of a light $\left(\bar{\rho} \bar{h}=10^{-3}\right)$, medium $(\bar{\rho} \bar{h}=1)$ and heavy $(\bar{\rho} \bar{h}=10)$ beam. For each case, there is an early transient stage consisting of oscillations of the beam, but these decay as the beam approaches a steady state. Since the damping is supplied by the viscous dissipation in the fluid, the oscillations decay more rapidly for lighter beams. At very long times, the solutions for beam displacement and velocity approximate the exact steady state solutions. Since the steady state solution is quadratic in $x$, we expect the agreement to be close to exact. The table in Figure 10 gives the max-norm errors in the steady state solutions for the light, medium and heavy beams. As expected, the AMP scheme is found to be stable and accurate in all three cases with errors close to the double-precision machine epsilon. Finally, we note that for this problem the traditional partitioned scheme (without sub-time-step iterations) is found to be unstable when $\bar{\rho} \bar{h}$ is less than 5 (approximately).
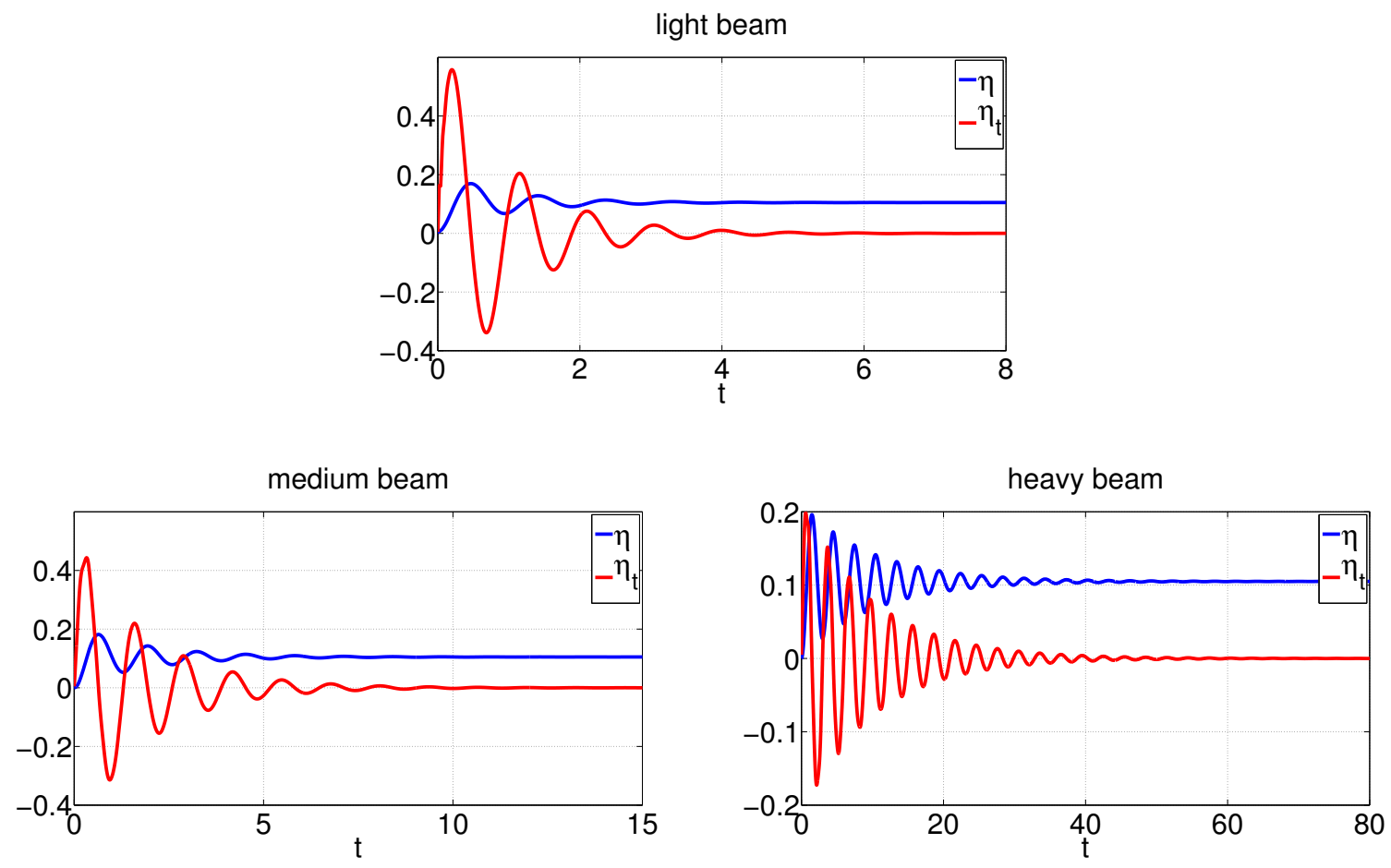

Figure 9: Beam displacement $\eta$ and velocity $\eta_{t}$ at its center, $x=1 / 2$, versus time for light $\left(\bar{\rho} \bar{h}=10^{-3}\right)$, medium $(\bar{\rho} \bar{h}=1)$ and heavy $(\bar{\rho} \bar{h}=10)$ beams.

\begin{tabular}{|c|c|c|c|c|c|c|}
\hline $\bar{\rho} \bar{h}$ & $t_{\mathrm{ss}}$ & $E_{j}^{(p)}$ & $E_{j}^{\left(v_{1}\right)}$ & $E_{j}^{\left(v_{2}\right)}$ & $E_{j}^{(\eta)}$ & $E_{j}^{\left(\eta_{t}\right)}$ \\
\hline $1 \mathrm{e}-3$ & 30 & $1.50 \mathrm{e}-12$ & $7.06 \mathrm{e}-14$ & $2.25 \mathrm{e}-13$ & $2.00 \mathrm{e}-15$ & $2.25 \mathrm{e}-13$ \\
\hline 1 & 50 & $4.53 \mathrm{e}-12$ & $3.73 \mathrm{e}-14$ & $7.15 \mathrm{e}-13$ & $1.80 \mathrm{e}-13$ & $7.15 \mathrm{e}-13$ \\
\hline 10 & 250 & $4.19 \mathrm{e}-12$ & $3.84 \mathrm{e}-14$ & $2.63 \mathrm{e}-12$ & $9.08 \mathrm{e}-14$ & $2.63 \mathrm{e}-12$ \\
\hline
\end{tabular}

Figure 10: Maximum-norm errors at near steady state when $t=t_{\mathrm{ss}}$ for light $\left(\bar{\rho} \bar{h}=10^{-3}\right)$, medium $(\bar{\rho} \bar{h}=1)$ and heavy $(\bar{\rho} \bar{h}=10)$ beams.

\subsection{Verification results for a beam with finite thickness}

For the case of FSI problems involving beams with finite thickness, we first consider solutions of two further benchmark problems to verify the stability and accuracy of the AMP scheme for this more difficult 
configuration. All numerical solutions described in this section, and in the subsequent sections, are computed using deforming composite grids and the beam solver based on finite elements. As part of the verification tests, we consider both the AMP-PBA and AMP-PBF implementations of the AMP interface conditions. For both benchmark problems, the beam has constant nonzero thickness $\bar{h}$ and separates fluid domains on either side.

\subsubsection{Dynamic motion of a flat beam separating two fluid chambers}

We consider a beam separating two initially rectangular fluid domains. The beam is assumed to have finite thickness $\bar{h}$, and is chosen to initially lie along the $x$-axis and occupy the domain $\bar{\Omega}(0)=[-1,1] \times$ $[-\bar{h} / 2, \bar{h} / 2]$. At time $t=0$, the lower fluid domain is the rectangular domain $\Omega_{-}(0)=[-1,1] \times\left[H^{-},-\bar{h} / 2\right]$, while the upper fluid domain is $\Omega_{+}(0)=[-1,1] \times\left[\bar{h} / 2, H^{+}\right]$. For this first test problem, we assume that the beam satisfies a sliding boundary condition $\eta_{s}=\bar{E} \bar{I} \eta_{s s s}=0$ at each end, and that the fluid satisfies slip-wall conditions along the vertical boundaries. At the top and bottom fluid boundaries, we assume that $v_{1}=0$ and $p=P_{ \pm}(t)$, where $P_{ \pm}(t)$ are specified time-dependent pressures. For this configuration, an exact solution can be found for which the beam moves vertically, but remains flat. In the fluid, the horizontal component of the velocity is zero, while the vertical component of the velocity and the pressure depend only on $y$ and $t$. This solution, albeit relatively simple, provides a good test of the stability of the AMP scheme for finite-thickness beams and for the two implementations of the AMP interface conditions.

Working through the equations governing the fluids in each chamber, assuming no dependence on $x$ and that the densities in both chambers are equal and given by $\rho$, and using the kinematic matching conditions at the top and bottom surfaces of the beam, we find that $v_{1}=0$ and $v_{2}=d \eta / d t$ in both fluid chambers, and

$$
p(\mathbf{x}, t)=\left\{\begin{array}{lll}
P_{-}(t)-\rho\left(y-H^{-}\right) \frac{d^{2} \eta}{d t^{2}}, & \mathbf{x} \in \Omega_{-}(t)=[-1,1] \times\left[H^{-}, \eta-\bar{h} / 2\right], & t>0, \\
P_{+}(t)-\rho\left(y-H^{+}\right) \frac{d^{2} \eta}{d t^{2}}, & \mathbf{x} \in \Omega_{+}(t)=[-1,1] \times\left[\eta+\bar{h} / 2, H^{+}\right], & t>0 .
\end{array}\right.
$$

The equation governing an EB beam with all terms involving spatial derivatives set to zero is

$$
\bar{\rho} \bar{h} \frac{d^{2} \eta}{d t^{2}}=-\bar{K}_{0} \eta-\bar{K}_{1} \frac{d \eta}{d t}+p(x, \eta-\bar{h} / 2, t)-p(x, \eta+\bar{h} / 2, t), \quad x \in[-1,1], \quad t>0,
$$

Using the expressions for the fluid pressures in (78), we find that the vertical displacement of the (flat) beam satisfies

$$
(\bar{\rho} \bar{h}+\rho H) \frac{d^{2} \eta}{d t^{2}}+\bar{K}_{1} \frac{d \eta}{d t}+\bar{K}_{0} \eta=\Delta P(t), \quad t>0,
$$

where $H=H^{+}-H^{-}-\bar{h}$ is the total depth of the fluid chambers and $\Delta P(t)=P_{-}(t)-P_{+}(t)$ is the difference between the applied pressures. We observe that the displacement of the beam satisfies a standard spring equation with mass given by the sum of the beam mass, $\bar{\rho} \bar{h}$, and the fluid added mass, $\rho H$, damping given by $\bar{K}_{1}$, spring constant given by $\bar{K}_{0}$, and a time-dependent external forcing given by $\Delta P(t)$. Exact solutions for $\eta(t)$ satisfying (80) for chosen initial conditions are easily found. For example, if $\eta=d \eta / d t=0$ at $t=0$, and if $\bar{K}_{1}=0$ and $\Delta P(t)$ is constant, then

$$
\eta(t)=\frac{\Delta P}{K_{0}}\{(1-\cos (\omega t)\}, \quad t>0,
$$

where the frequency of the oscillating beam is given by

$$
\omega=\sqrt{\frac{K_{0}}{\bar{\rho} \bar{h}+\rho H}} .
$$

Numerical solutions of this FSI problem are computed using the full DCG methodology as discussed in Section 7.1. The composite grid $\mathcal{G}_{\text {fp }}^{(j)}$ for the geometry is shown in the left plot of Figure 13, and consists of a background Cartesian grid and a body-fitted grid adjacent to the beam surface for each fluid chamber. The two body-fitted grids are generated independently using the hyperbolic grids generator, and each has 6 

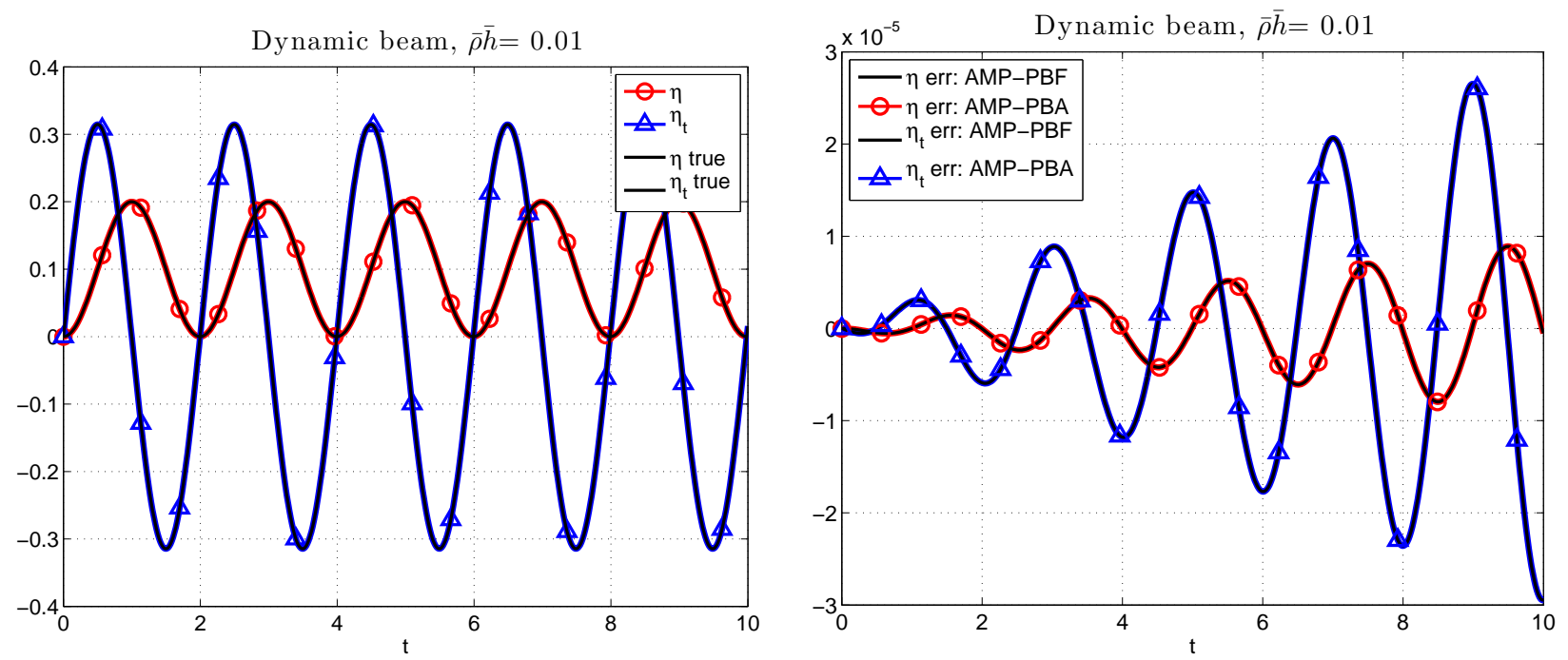

Figure 11: Oscillating beam between two fluids for $\bar{\rho} \bar{h}=0.01$. Left: beam vertical displacement, $\eta$, and velocity, $\eta_{t}$, compared to the exact solution. Right: errors in $\eta$ and $\eta_{t}$ for the AMP-PBF (predict beam internal force) and AMP-PBA (predict beam acceleration) schemes. Results are for the coarse grid $\mathcal{G}_{\mathrm{fp}}^{(2)}$.

\begin{tabular}{|c|c|c|c|c|c|c|c|c|}
\hline \multicolumn{1}{|c|}{ Oscillating beam, $\bar{\rho} \bar{h}=10$} \\
\hline$h_{j}$ & $E_{j}^{(p)}$ & $r$ & $E_{j}^{(\mathbf{v})}$ & $r$ & $E_{j}^{(\eta)}$ & $r$ & $E_{j}^{\left(\eta_{t}\right)}$ & $r$ \\
\hline $1 / 20$ & $5.5 \mathrm{e}-6$ & & $1.2 \mathrm{e}-5$ & & $7.9 \mathrm{e}-6$ & & $9.6 \mathrm{e}-6$ & \\
\hline $1 / 40$ & $6.7 \mathrm{e}-7$ & 8.3 & $1.2 \mathrm{e}-6$ & 10.2 & $8.8 \mathrm{e}-7$ & 9.0 & $1.1 \mathrm{e}-6$ & 8.9 \\
\hline $1 / 80$ & $6.2 \mathrm{e}-8$ & 10.8 & $1.1 \mathrm{e}-7$ & 10.5 & $8.8 \mathrm{e}-8$ & 10.0 & $1.1 \mathrm{e}-7$ & 10.0 \\
\hline $1 / 160$ & $4.0 \mathrm{e}-9$ & 15.2 & $7.5 \mathrm{e}-9$ & 14.6 & $6.1 \mathrm{e}-9$ & 14.4 & $7.5 \mathrm{e}-9$ & 14.5 \\
\hline rate & 3.47 & & 3.52 & & 3.43 & & 3.43 & \\
\hline
\end{tabular}

\begin{tabular}{|c|c|c|c|c|c|c|c|c|}
\hline \multicolumn{10}{|c|}{ Oscillating beam, $\bar{\rho} \bar{h}=0.01$} \\
\hline$h_{j}$ & $E_{j}^{(p)}$ & $r$ & $E_{j}^{(\mathbf{v})}$ & $r$ & $E_{j}^{(\eta)}$ & $r$ & $E_{j}^{\left(\eta_{t}\right)}$ & $r$ \\
\hline $1 / 20$ & $3.1 \mathrm{e}-4$ & & $1.7 \mathrm{e}-4$ & & $4.8 \mathrm{e}-5$ & & $9.1 \mathrm{e}-5$ & \\
\hline $1 / 40$ & $1.3 \mathrm{e}-5$ & 22.8 & $5.1 \mathrm{e}-6$ & 34.1 & $2.1 \mathrm{e}-6$ & 22.8 & $4.8 \mathrm{e}-6$ & 19.0 \\
\hline $1 / 80$ & $9.2 \mathrm{e}-7$ & 14.6 & $3.3 \mathrm{e}-7$ & 15.3 & $1.4 \mathrm{e}-7$ & 14.6 & $3.3 \mathrm{e}-7$ & 14.5 \\
\hline $1 / 160$ & $5.8 \mathrm{e}-8$ & 15.9 & $2.1 \mathrm{e}-8$ & 16.1 & $8.9 \mathrm{e}-9$ & 16.0 & $2.1 \mathrm{e}-8$ & 15.9 \\
\hline rate & 4.10 & & 4.30 & & 4.10 & & 4.02 & \\
\hline
\end{tabular}

Figure 12: Oscillating beam between two fluids. Maximum errors and estimated convergence rates at $t=0.7$ computed using the AMP scheme for a heavy beam, $\bar{\rho} \bar{h}=10$ and a light beam $\bar{\rho} \bar{h}=0.01$. For this problem the convergence rates are close to four since the errors are primarily due to the beam solver which is fourth-order accurate in space.

grids lines in the normal direction (so that the grids becomes thinner as the composite grid is refined). The grid spacings in each coordinate direction are approximately equal for all component grids and chosen to be $h_{j}=1 /(10 j)$. As before, the index $j$ determines the resolution of the grid.

The parameters for the EB beam in the numerical calculations are taken as $\bar{h}=0.1, \bar{K}_{0}=10, \bar{T}=10^{-1}$, $\bar{E} \bar{I}=10^{-3}, \bar{K}_{1}=\bar{T}_{1}=0$, and $\bar{\rho}$ varied to test light and heavy beams. The parameters for the fluid are $\rho=1, \mu=.02, H^{-}=-.5-\bar{h} / 2$, and $H^{+}=.5+\bar{h} / 2$ so that $H=1$. The pressure difference between the top and bottom was taken as $\Delta P=1$. Figure 11 shows the computed values of $\eta$ and $d \eta / d t$ compared to the exact solution for a light beam $\bar{\rho} \bar{h}=10^{-2}$ on the grid $\mathcal{G}_{\mathrm{fp}}^{(2)}$. The differences between the computed and 
exact solutions are nearly indistinguishable. Figure 11 also plots the errors in $\eta$ and $d \eta / d t$ for the AMP-PBF (predict beam internal force) and AMP-PBA (predict beam acceleration) schemes, and the results are again nearly indistinguishable. The tables in Figure 12 shows the max-norm errors and estimated convergence rates for a light beam, $\bar{\rho} \bar{h}=10^{-2}$, and a heavy beam, $\bar{\rho} \bar{h}=10$ at $t=0.7$. The maximum errors are converging close to fourth-order accuracy. Recall that the beam solver is fourth-order accurate in space and second-order order accurate in time but converges as fourth-order overall when the time-step scales as $\Delta t \propto h_{j}^{2}$, which is the case for the calculations presented here.

\subsubsection{Steady state beam separating two pressurized fluid chambers}

As a final benchmark test case for which an exact solution is available, we consider the long-time behavior of an EB beam separating two initially rectangular pressurized fluid chambers. The geometric configuration for this test case is the same as that considered in the previous test for a beam with finite thickness $\bar{h}$, but the boundary conditions on the vertical sides are changed so that the beam does not remain flat as time evolves. Here we assume no-slip boundary conditions for the fluid on the side walls, and take $\eta=\partial \eta / \partial s=0$ as clamped boundary conditions for the EB beam. The material properties of the beam are taken to be $\bar{h}=0.1, \bar{T}=0, \bar{E} \bar{I}=0.2, \bar{K}_{1}=5$ and $\bar{T}_{1}=0$, with $\bar{\rho}$ varied to consider light, medium and heavy beams. The properties of the fluid are $\rho=1, \mu=.05, P_{+}=0$ and $P_{-}(t)=P_{0} R(t)$, with $P_{0}=1$, where $R(t)$ is a smooth ramp function given by

$$
R(t)= \begin{cases}(35+(-84+(70-20 t) t) t) t^{4}, & \text { for } 0 \leq t \leq 1 \\ 1, & \text { for } t>1\end{cases}
$$

The ramp function satisfies $R=R^{\prime}=R^{\prime \prime}=R^{\prime \prime \prime}=0$ at $t=0$ and has three continuous derivatives at $t=1$. Thus, the applied pressure in the lower chamber varies smoothly from $P_{-}(0)=0$ to $P_{-}(1)=P_{0}$, and remains equal to $P_{0}$ for $t>1$. At $t=0$, the fluid in both chambers is at rest, and the displacement and velocity of the beam are both zero.

As in the FSI problem considered in Section 8.1.2, the beam oscillates in response to the nonzero applied fluid pressure in the bottom chamber, but then approaches a steady state solution as time increases due to the viscous dissipation in the fluid and damping in the beam. At steady state, the velocity in both fluid chambers is zero and the fluid pressures are uniform as before, but now for the beam parameters considered here the displacement of the beam is given by

$$
\eta=\frac{P_{0}}{24 \bar{E} \bar{I}}\left(1-x^{2}\right)^{2}, \quad x \in[-1,1]
$$
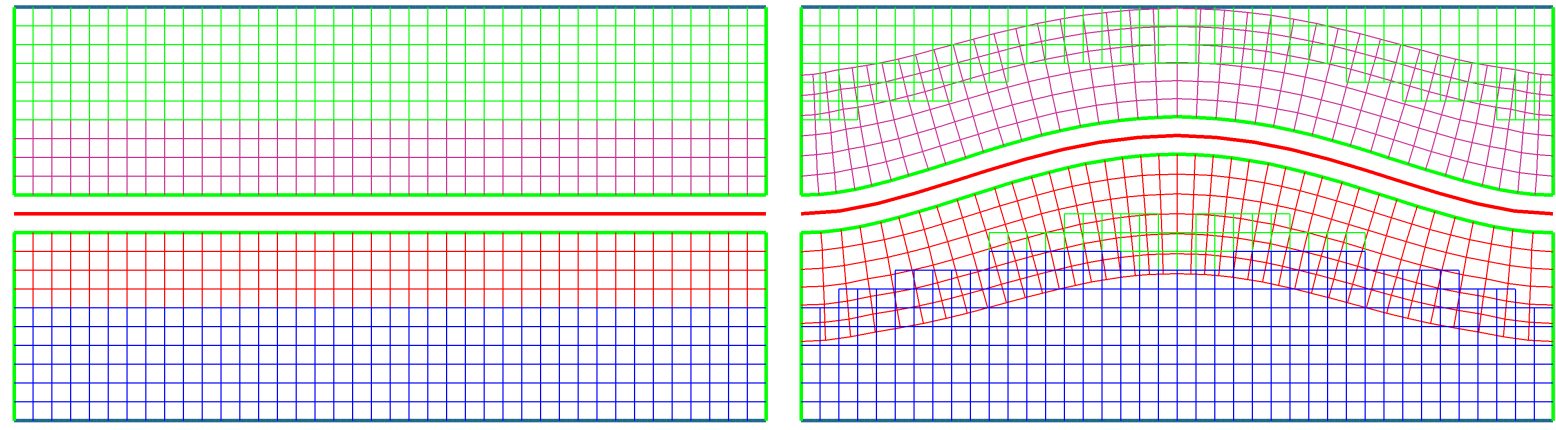

Figure 13: Composite grids for the partitioned chambers: coarse grid $\mathcal{G}_{\mathrm{fp}}^{(2)}$ at $t=0$ (left) and at $t=10$ (right). The solution at $t=10$ has nearly reached steady state.

Numerical solutions are computed using the composite grid $\mathcal{G}_{\mathrm{fp}}^{(j)}$, as before, with grid spacings approximately equal to $h_{j}=1 /(10 j)$. Figure 13 shows a representative grid, $\mathcal{G}_{\mathrm{fp}}^{(2)}$, at $t=0$ (left) and at $t=10$ (right) computed for the case $\bar{\rho} \bar{h}=0.1$. The solution at $t=10$ has nearly reached steady state. The maximum error 
in the beam displacement compared to the steady state solution is approximately $4 \times 10^{-7}$ at $t=10$, and reaches $10^{-15}$ by $t=50$. Figure 14 shows the motion of the beam midpoint for different beam densities and different algorithmic options with a smaller damping coefficient of $\bar{K}_{1}=1$. The top-left graph in the figure shows the behavior of the displacement, $\eta(0, t)$, and velocity, $\eta_{t}(0, t)$, as functions of time. Results are shown for a light beam, $\bar{\rho} \bar{h}=0.01$, and a medium beam, $\bar{\rho} \bar{h}=1$, computed on a coarse grid $\mathcal{G}_{\mathrm{fp}}^{(2)}$ and a finer grid $\mathcal{G}_{\mathrm{fp}}^{(4)}$. The light beam is excited more rapidly than the medium, and undergoes more rapid oscillations. We also observe that the time histories computed using the coarse and fine grid are nearly indistinguishable for both beams. The top-right graph in Figure 14 compares results from the AMP scheme and the traditional partitioned scheme with sub-iterations (TP-SI) for the case $\bar{\rho} \bar{h}=0.01$. Added-mass effects are relatively large for this light beam requiring a relaxation parameter in the TP-SI iteration of $\omega=0.1$. The TP-SI scheme required an average of approximately 77 sub-iterations per time step to achieve a sub-iteration convergence tolerance of $10^{-5}$. The results from the AMP and TP-SI schemes are in good agreement, but the computational cost for the TP-SI scheme is significantly larger.

The dynamic beam problem provides a good test to compare the behavior of the two AMP variations described in Section 7.2 for the finite-element based beam solver. It is our experience for this problem, and others, that for very light beams the AMP-PBA (predict beam acceleration) algorithm tends to be more robust (e.g. requires fewer or no smoothing iterations) than the AMP-PBF (predict beam internal force). The AMP-PBF approach requires the evaluation of the third spatial-derivative of the finite element beam displacement and this can be sensitive, especially near the ends of the beam where one-sided approximations are used (as might be expected). The bottom graph in Figure 14 compares results obtained using the AMPPBA and AMP-PBF for a moderately light beam with $\bar{\rho} \bar{h}=0.1$. In this case the results obtained using both AMP variations are in excellent agreement. However, for a very light beam, $\bar{\rho} \bar{h}=0.01$, the AMP-PBF variation has some difficulty and shows poor behavior near the ends of the beam where the acceleration of the beam varies very rapidly during the startup phase when the pressure forcing is turned on. It is entirely possible that this sub-optimal behavior of the AMP-PBF variation could be rectified with further refinements to the algorithm, but we have not pursued this since the alternative AMP-PBA variation behaves well and is simpler to implement.

\subsection{Traveling-wave pressure-pulse in an elastic tube}

The propagation of a pressure pulse through a two-dimensional elastic tube can be used as a model for the flow of blood in a large artery or vein following the problem proposed in [34], and later considered in [13, 21] to study the stability of partitioned schemes. Added-mass effects are important in this application since the density of the elastic wall is close to that of blood and also since the tube is long and thin. Traditional partitioned methods require many sub-iterations per time step to remain stable for this problem. The AMP algorithm is shown to provide stable results with no sub-iterations per time step.

The domain for the fluid is the channel $\Omega(t)=[0, L] \times[0, R(t)]$, where $L$ is the length of the channel and $R(t)$ is the distance from the axis of symmetry of the channel at $y=0$ and the elastic wall. We consider the case when $L=6$ and $R(0)=0.5$. The boundary conditions for the fluid domain are as follows. The bottom boundary is a slip-wall where $v_{2}=0$ and $\partial v_{1} / \partial y=0$. The top boundary is the flexible beam where the AMP interface conditions are applied. On the left boundary the tangential component of the velocity is set to zero, $v_{2}=0$, and the pressure is specified to be the time-dependent pulse

$$
p(0, y, t)= \begin{cases}p_{\max } \sin \left(\pi t / t_{\max }\right) & \text { for } 0 \leq t \leq t_{\max }, \\ 0 & \text { for } t>t_{\max },\end{cases}
$$

where $p_{\max }$ and $t_{\max }$ determine the magnitude and duration of the pulse (values given below). The right boundary is an outflow boundary where $p=0$ and the components of velocity are extrapolated.

The composite grid $\mathcal{G}_{\text {et }}^{(j)}$ for the fluid domain at various times is shown in Figure 15. The composite grid consists of a background Cartesian grid (shown in blue) together with a hyperbolic grid adjacent to the top interface (shown in green). The hyperbolic grid is generated with 6 grids lines in the normal direction and thus this boundary-fitted component grid becomes thinner as the composite grid is refined. The grid spacing is chosen to be $h_{j}=1 /(10 j)$ approximately.

We perform simulations of the elastic tube problem for two choices of the parameters of the fluid and the beam, identified as Parameter Sets I and II. The parameter sets are based on the parameters chosen in 

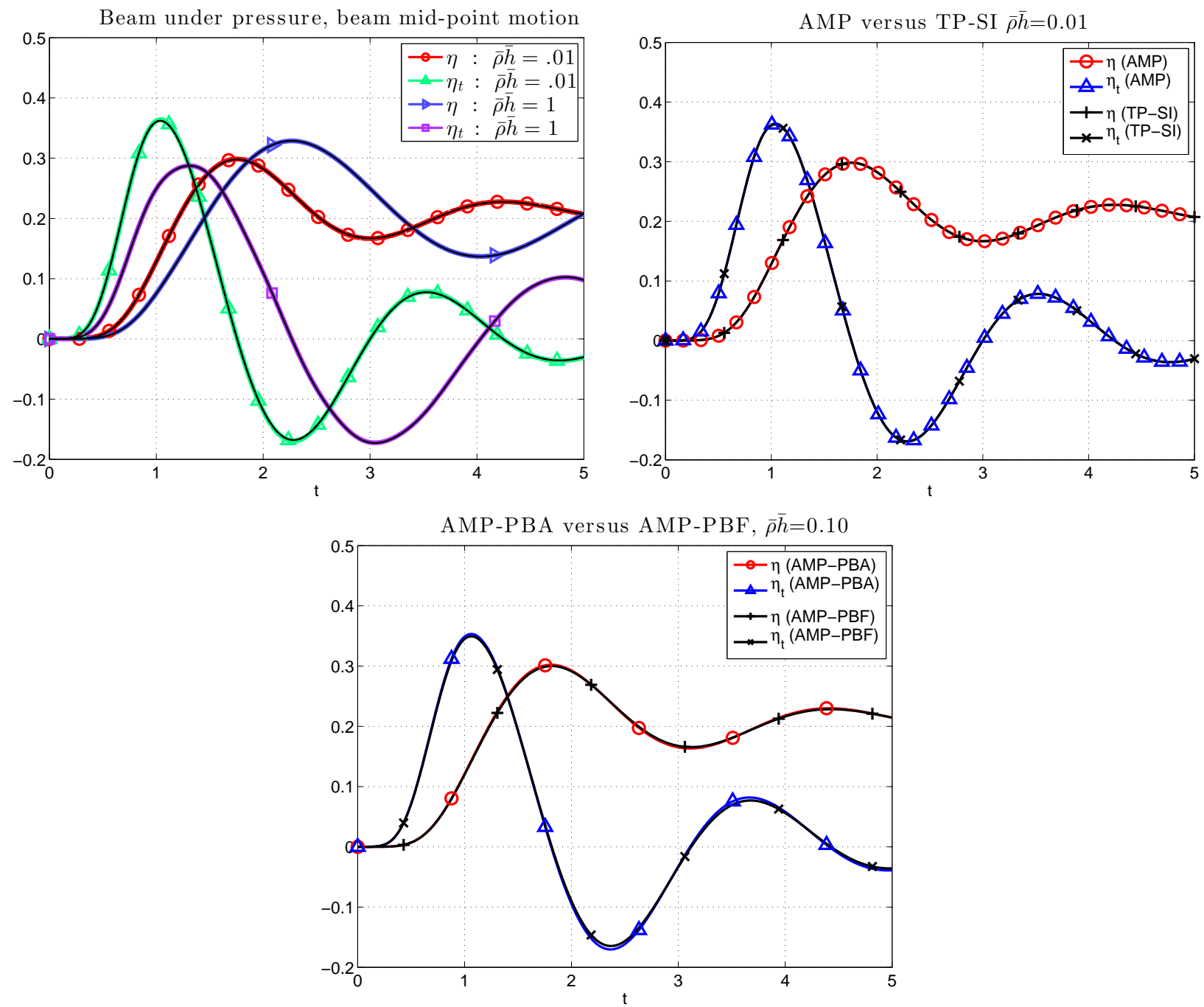

Figure 14: Beam under pressure, motion of the beam mid-point. Top left: $\eta$ and $\eta_{t}$ for a light $(\bar{\rho} \bar{h}=0.01)$ and medium $(\bar{\rho} \bar{h}=1)$ beam using a coarse grid $\mathcal{G}_{\mathrm{fp}}^{(2)}$ (colored curves) and a finer grid $\mathcal{G}_{\mathrm{fp}}^{(4)}$ (black curves). Top right: AMP versus the TP-SI scheme for the light beam, $\bar{\rho} \bar{h}=0.01$. Bottom: A comparsion of the two AMP schemes for coupling to the finite-element beam solver on grid $\mathcal{G}_{\mathrm{fp}}^{(4)}$ for $\rho \bar{h}=0.1$.

Fernandez et al. [21], but with some differences as described below. For both choices, the beam is taken to be a generalized string model with $\bar{E} \bar{I}=0$ (i.e. no fourth-order spatial derivative term in the beam equation). The AMP-PBA (predict-beam-acceleration) variation is used for all calculations in this section and the projected interface velocity is smoothed with three iterations of the fourth-order filter, as described in Section 7.3.

\subsubsection{Parameter Set I}

Dimensionless parameters for this set are taken to be $\rho=1, \mu=10^{-2}, p_{\max }=2$ and $t_{\max }=0.75$ for the fluid, and $\bar{\rho} \bar{h}=0.11, \bar{T}=5 / 6 \approx .83333, \bar{K}_{0}=40 / 3 \approx 13.333, \bar{K}_{1}=\bar{\rho} \bar{h} / 100$ and $\bar{T}_{1}=5 / 60 \approx .08333$ for the beam. The term with coefficient $\bar{K}_{0}$ acts as a linear restoring force while the term with coefficient $\bar{T}_{1}$ is a visco-elastic damping term that tends to smooth high-frequency oscillations in space. The parameters used for this first set are slightly different than the ones used in [21]. Our parameters give a larger amplitude 

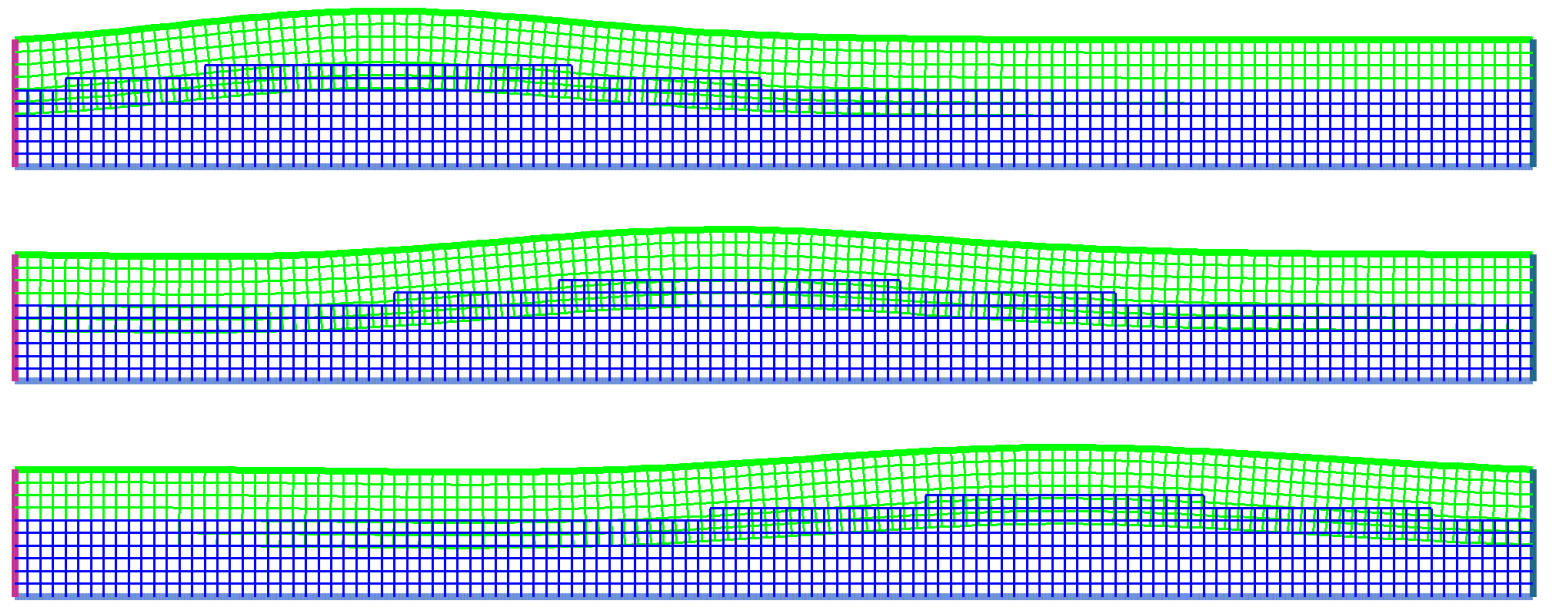

Figure 15: Composite grids for the traveling-wave pressure-pulse in a flexible tube: coarse grid $\mathcal{G}_{\mathrm{et}}^{(2)}$ at times $t=1.0$, 1.5 and 2.0 .

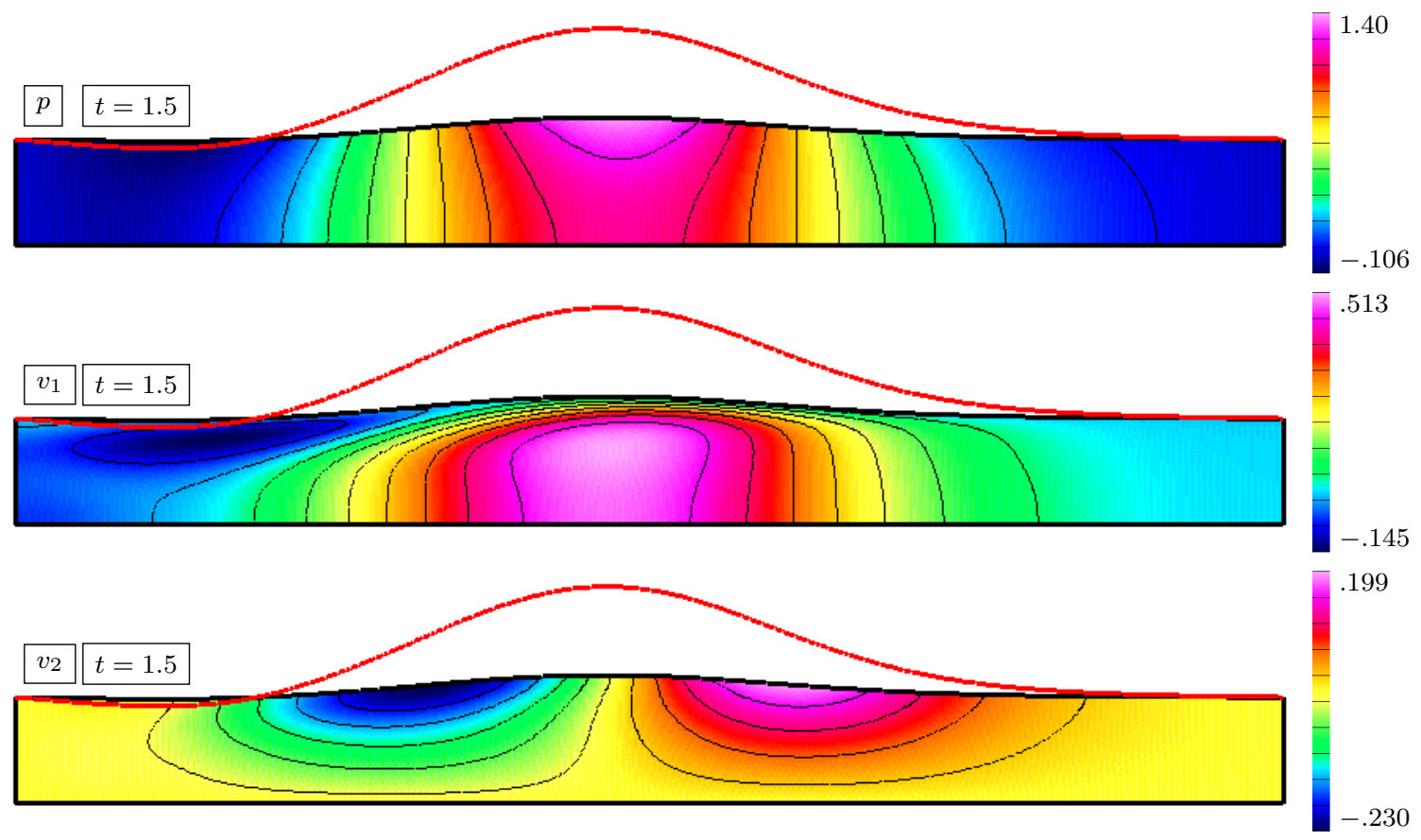

Figure 16: Parameter set I: Traveling-wave pressure-pulse in a flexible tube. Contours of $p, v_{1}$ and $v_{2}$ at $t=1.5$ computed on grid $\mathcal{G}_{\mathrm{et}}^{(8)}$. For clarity, the top surface curve is alse is shown in red with the displacement amplified by a factor of 5 .

of the deformation of the beam and a thicker boundary layer in the fluid near the surface of the beam. A larger deformation of the beam is a more severe test of our DCG approach, and a thicker boundary layer enables grid-convergence tests demonstrating second-order accuracy using coarser grids. (The choice of viscosity used in [21] requires a very fine grid to resolve the very thin boundary layer, see Parameter Set II in Section 8.3.2 below). We also note that the fluid tractions and velocities are transferred directly on the beam reference curve for the simulations described in [21], whereas our approach applies these quantities on the beam surface as described in Section 7.2. In order to approximate a beam of negligible thickness with our approach, we choose a small value for the beam thickness of $\bar{h}=10^{-3}$. 

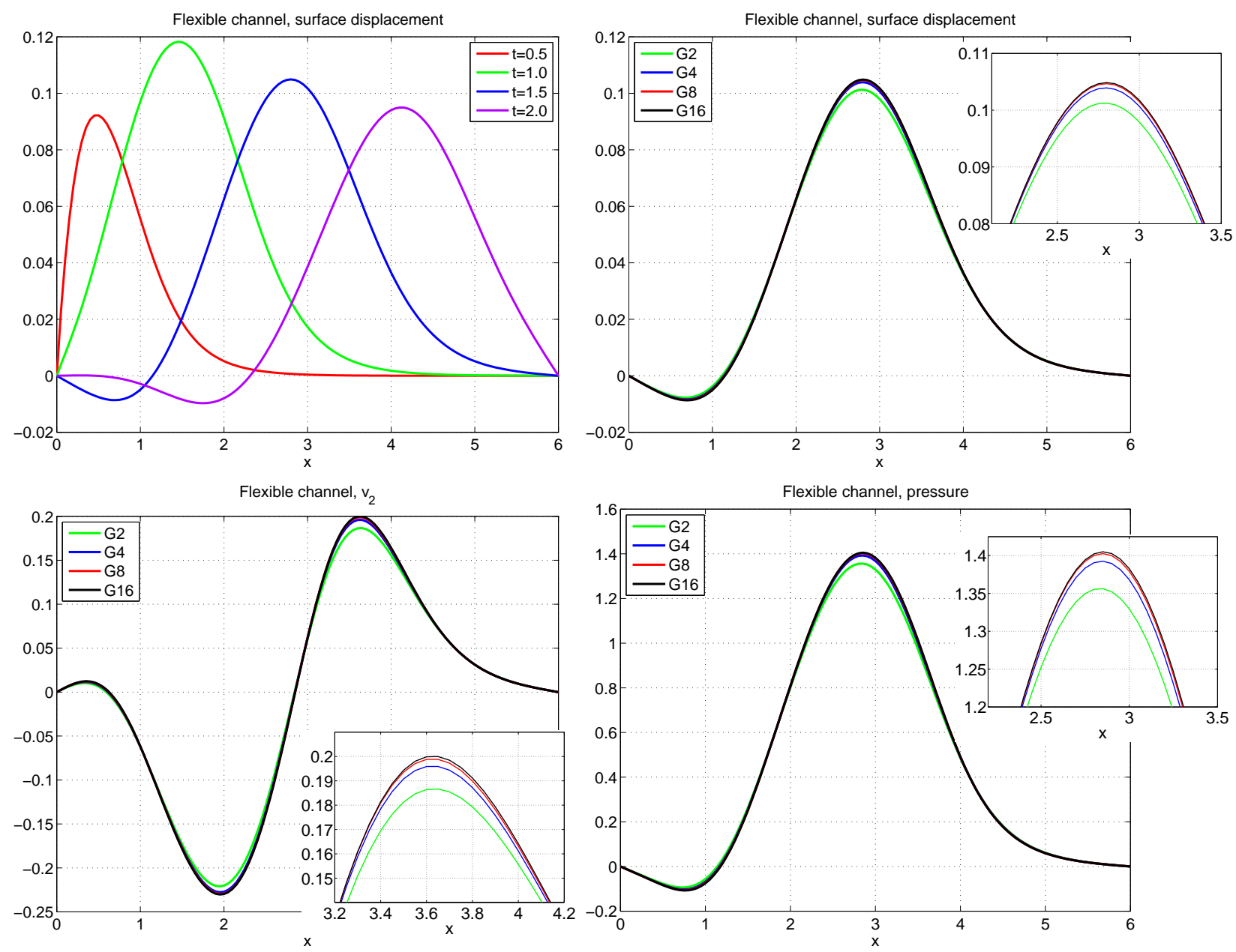

Figure 17: Traveling-wave pressure-pulse in a flexible tube. Top left: surface displacement over time showing the formation and propogation of the pulse. Top right: surface displacement at $t=1.5$ for grids $\mathcal{G}_{\text {et }}^{(k)}, k=2,4,8,16$ showing the convergence of the surface as the grid is refined. Bottom left and lower right: convergence of $v_{2}$ and $p$, respectively, as the grid is refined.

Figure 16 shows shaded contours of the fluid pressure and the components of the fluid velocity at $t=1.5$. The pressure pulse at this time has created a bulge in the flexible channel near its half-length, and for clarity the centerline-curve of the beam on the top surface is drawn as a red curve with the displacement amplified by a factor of 5 . The traveling pressure pulse is seen to consist of a localized high pressure region where the beam displacement is positive. The pressure is largest at the surface near the maximum displacement of the beam. The horizontal component of the velocity is generally large and positive within the pulse with a boundary layer near the top surface to match the no-slip condition there. The vertical component of velocity is positive ahead of the pulse and negative behind it confirming that the pulse is traveling in the positive $x_{1}$ direction.

The top left plot in Figure 17 shows the evolution of the shape of the surface at four different times. The surface is initially displaced upwards on the left side due to the high pressure at inflow (82) which acts over the time interval $\left[0, t_{\max }\right]$, where $t_{\max }=0.75$. The surface pulse then propagates to the right and slowly decays in amplitude. The top right plot shows the beam displacement at $t=1.5$ computed using grids with increasing resolution. The solutions are seen to converge with the curves on two finest grids being nearly indistinguishable. The two plots on the bottom of Figure 17 show the grid convergence of the vertical component of the fluid velocity and the fluid pressure at the surface of the beam at $t=1.5$. Here, we also observe excellent convergence of these two fluid variables. 
To provide a more quantitative estimate of the solution accuracy, a self-convergence study is performed. Given a sequence of three or more grids of increasing resolution, a posteriori estimates of the errors and convergence rates can be computed using the Richardson extrapolation procedure described in [35]. These self-convergence estimates assume that the numerical solution converges to a limiting solution, but does not assume that some chosen fine-grid solution is the exact solution. A posteriori estimates computed in this way are given in the table in Figure 18 for four grids of increasing resolution (the convergence rate computation uses the three finest resolutions). The table provides estimates of the max-norm errors for various components of the solutions and the corresponding estimates of the convergence rates. We observe that all solution components are converging with approximately second-order accuracy. Note that due to the manner in which rates are computed, the ratios of errors in the columns headed "r" are the average ratios and thus the same for each grid resolution.

\begin{tabular}{|c|c|c|c|c|c|c|}
\hline \multicolumn{7}{|c|}{ Traveling wave pressure-pulse } \\
\hline$h_{j}$ & $E_{j}^{(p)}$ & $r$ & $E_{j}^{\left(v_{1}\right)}$ & $r$ & $E_{j}^{\left(v_{2}\right)}$ & $r$ \\
\hline $1 / 20$ & $5.0 \mathrm{e}-2$ & & $5.4 \mathrm{e}-2$ & & $1.4 \mathrm{e}-2$ & \\
\hline $1 / 40$ & $1.4 \mathrm{e}-2$ & 3.6 & $1.4 \mathrm{e}-2$ & 3.8 & $3.6 \mathrm{e}-3$ & 4.0 \\
\hline $1 / 80$ & $3.8 \mathrm{e}-3$ & 3.6 & $3.8 \mathrm{e}-3$ & 3.8 & $9.1 \mathrm{e}-4$ & 4.0 \\
\hline $1 / 160$ & $1.0 \mathrm{e}-3$ & 3.6 & $1.0 \mathrm{e}-3$ & 3.8 & $2.3 \mathrm{e}-4$ & 4.0 \\
\hline rate & 1.87 & & 1.91 & & 1.99 & \\
\hline
\end{tabular}

Figure 18: Traveling wave pressure-pulse (parameter set I): Maximum errors and estimated convergence rates at $t=1.5$ computed using the AMP scheme.

Figure 19 compares results from the AMP scheme with the traditional-partitioned scheme with sub-timestep iterations (TP-SI) on the coarse grid $\mathcal{G}_{\mathrm{et}}^{(2)}$. The number of sub-iterations per time step required for the TP-SI scheme is approximately 15 on average, although near the start of the run almost 250 iterations are needed. (A convergence tolerance of $10^{-4}$ on the sub-iterations is used.) A simple under-relaxed fixed-point iteration is used with the value for the under-relaxation parameter taken as $\omega=0.025$ (the fact that such a small value is needed indicates that the problem has large added-mass effects). The solutions from the AMP and TP-SI are in excellent agreement.

\subsubsection{Parameter Set II}

The parameter values for this set match those of Fernandez et al. [21] so that a direct comparison of the results can be made. We prefer to work with dimensionless parameters obtained by scaling with the reference length $L_{0}=1 \mathrm{~cm}$, velocity $U_{0}=10^{2} \mathrm{~cm} / \mathrm{s}$, density $\rho_{0}=1 \mathrm{gm} / \mathrm{cm}^{3}$, and time $T_{0}=L_{0} / U_{0}=10^{-2} \mathrm{~s}$. These scalings give the corresponding dimensionless parameters $\rho=1, \mu=3.5 \times 10^{-4}, p_{\max }=2$ and $t_{\max }=0.5$ for the fluid, and $\bar{\rho} \bar{h}=0.11, \bar{T}=2.5, \bar{K}_{0}=40, \bar{K}_{1}=1.1 \times 10^{-3}$ and $\bar{T}_{1}=0.25$ for the beam. As in the previous set of parameters, the product $\bar{\rho} \bar{h}=0.11$ is fixed but $\bar{h}=10^{-3}$ and $\bar{\rho}=110$ are chosen to reduce the effects of a finite-width beam.

Figure 20 compares the surface displacement of the beam at $t=1.5$ computed with different grids using the AMP-PBA algorithm. The composite grids used in these calculations are the same as those used in the previous section. We observed that the surface displacement appears to be converging in a manner consistent with second-order accuracy. The figure also shows the results from Fernandez et al. [21] using a first-order accurate monolithic scheme on a grid with spacing $h=1 / 320$ (equal to the spacing on grid $\mathcal{G}_{\mathrm{et}}^{(32)}$ ). Although the monolithic results do not exactly match the fine grid AMP results, we note that the monolithic results do not yet appear to be grid converged since they differ by a fair amount from the monolithic results on a grid coarsened by a factor of 2 , see [21].

Figure 21 shows shaded contours and surface displacements of the solution at $t=1.0$ computed using the AMP algorithm. We note that the behavior of the solution is qualitatively similar to that observed previously for Parameter Set I. The contour plot of the horizontal component of velocity, $v_{1}$, however, shows the very thin boundary layer that forms near the interface. 

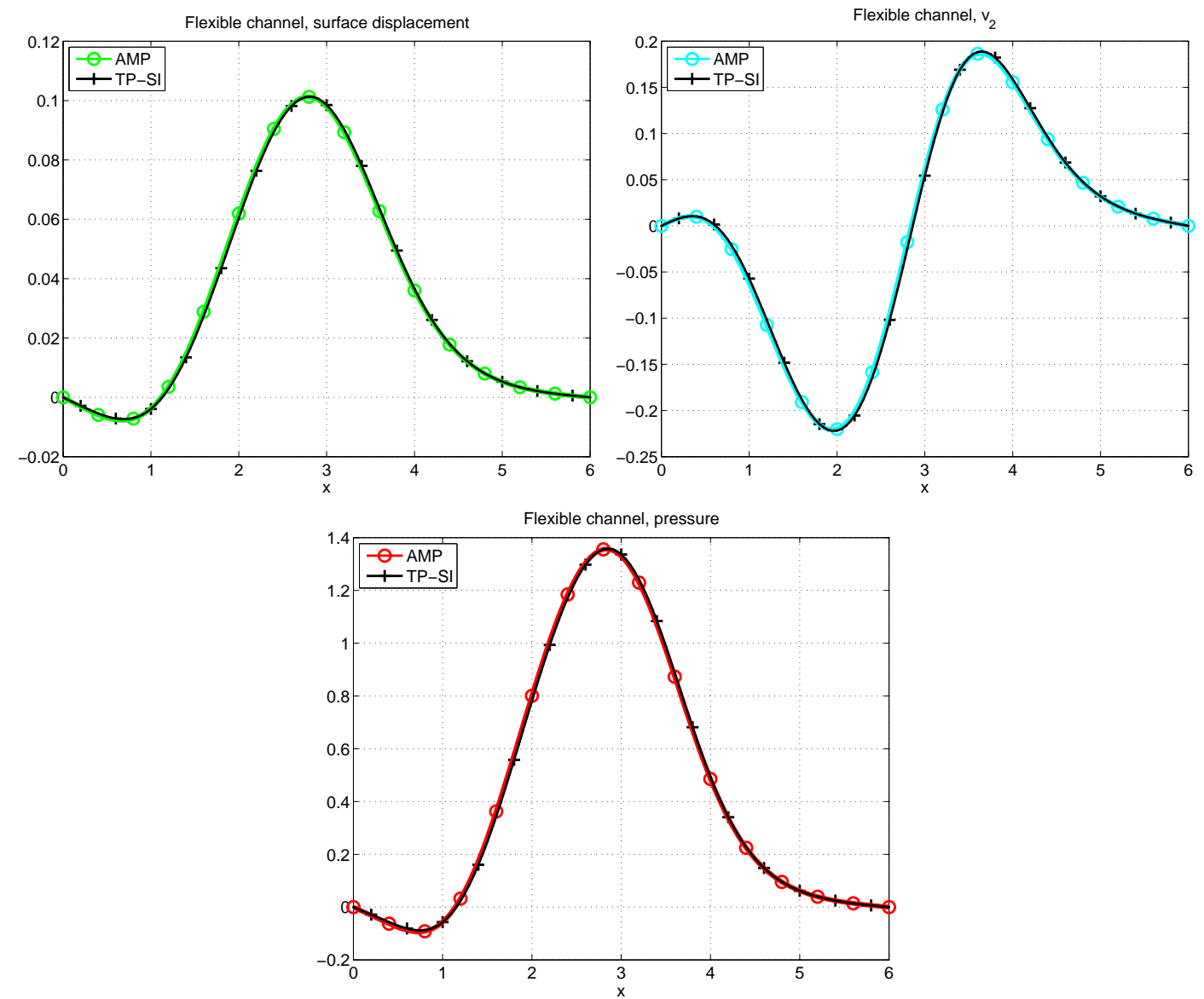

Figure 19: Traveling-wave pressure-pulse in a flexible tube. A comparison between the AMP scheme and the TP scheme with sub-time-step iterations at $t=1.5$ with grid $\mathcal{G}_{\mathrm{et}}^{(2)}$. The surface displacement, velocity $v_{2}$ and pressure are shown (with markers placed every 8 grid points). The results of the two schemes are seen to be nearly indistinguishable.

\subsection{Beam in a cross flow}

As a final demonstration of our AMP algorithm, we consider flow past a flexible beam that partially blocks a fluid channel as shown in Figure 22. A flow from left to right causes the beam to initially bend to the right and then oscillate. Eventually the flow approaches a steady-state consisting of a long recirculation region downstream of the deflected beam. The computed solution from the AMP scheme for a light beam is compared with the solution obtained using the TP-SI scheme. Results for beams of different densities are also shown and used to estimate the added mass for this configuration.

The geometry of the problem is illustrated in Figure 22 and consists of an initially vertical beam, with length $\bar{l}=1$, that extends into a rectangular fluid channel of height $y_{b}=2$ and overall dimensions $\left[x_{a}, x_{b}\right] \times\left[y_{a}, y_{b}\right]=[-3,8] \times[0,2]$. The thickness of the beam at its clamped base is $\bar{h}(0)=0.2$ (the beam reference line is located along the centerline) and this remains approximately constant along the beam until its rounded free end ${ }^{7}$. Note that while the beam reference-line (red curve in Figure 22) has an undeformed

7 The reason that the beam at the tip is rounded off, rather than having sharp corners, is so that a high-quality grid can be constructed that resolves the rapid variation of the solution around the tip. In addition, the solution and its derivatives will remain smooth near the rounded tip and this will give greater confidence that the numerical solution is converging to the true solution in this region. 


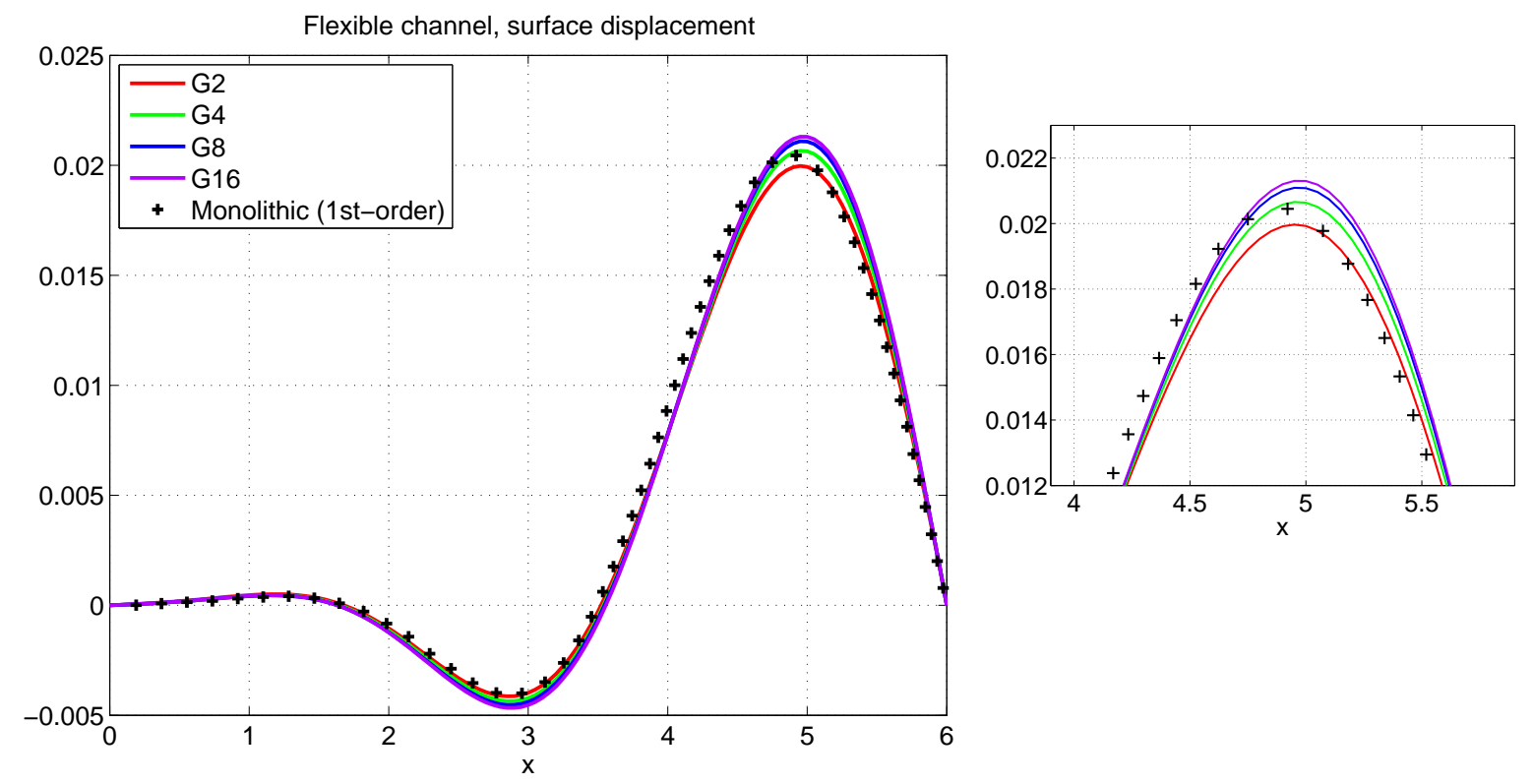

Figure 20: Paramater set II: Traveling-wave pressure-pulse in a flexible tube. Surface displacement as the grid are refined. Results for monolithic scheme are from Fernandez et.al. [21].

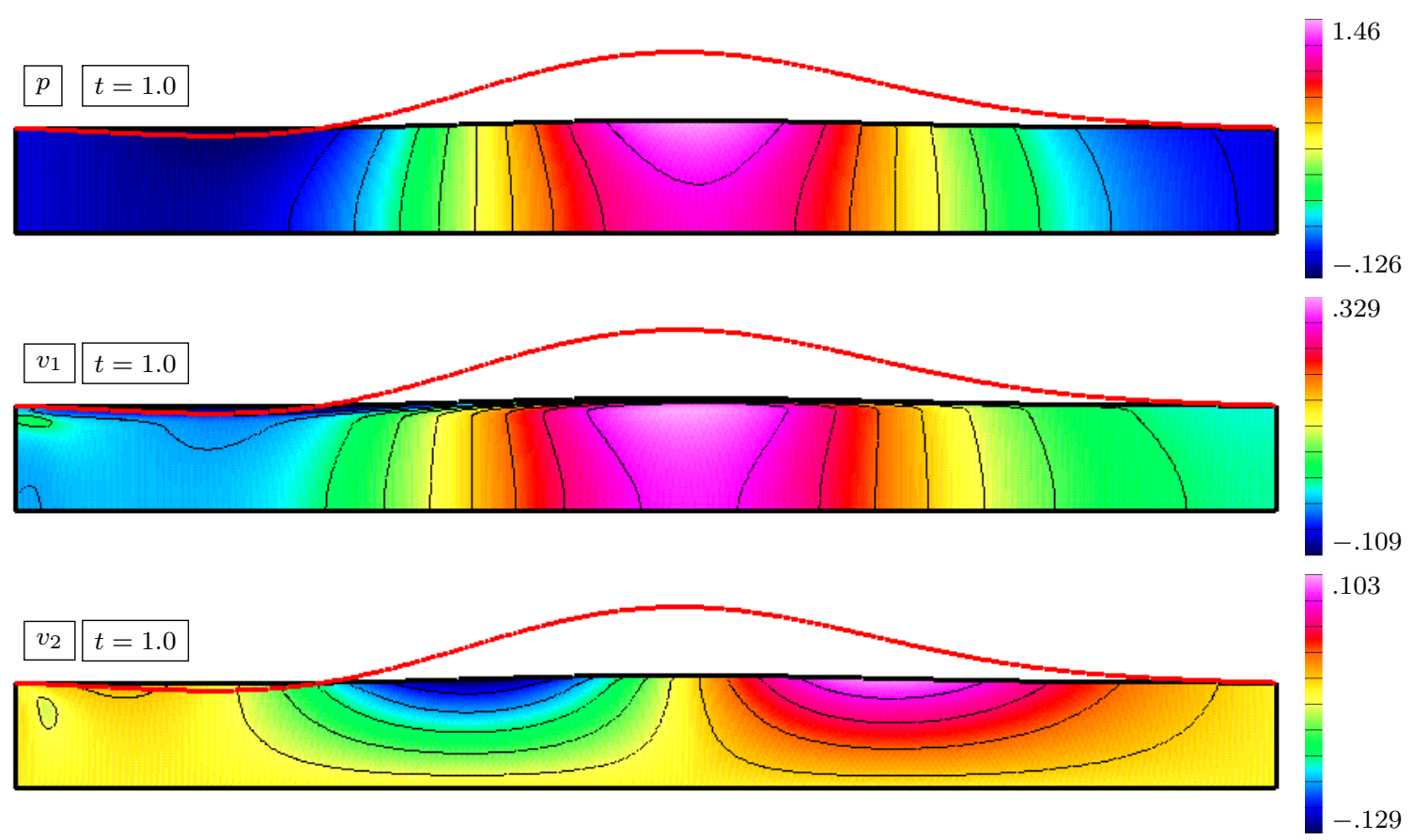

Figure 21: Parameter set II: Traveling-wave pressure-pulse in a flexible tube. Contours of $p, v_{1}$ and $v_{2}$ at $t=1.0$ computed on grid $\mathcal{G}_{\text {et }}^{(8)}$. For clarity, the top surface curve is alse is shown in red with the displacement amplified by a factor of 10 .

length of $\bar{l}=1$, the actual distance from the base of the beam to the rounded tip at the top is slightly longer ${ }^{8}$.

\footnotetext{
${ }^{8}$ The actual boundary and rounded end of the beam, and hence its thickness, is defined in terms of hyperbolic trigonometric functions through the SmoothedPolygon mapping [36]. The precise definition of the grid along with
} 
The base of the beam is at the origin of the computational domain, and the upstream vertical boundary of the channel is at $x_{a}=-3$ while the downstream boundary is at $x_{b}=8$. The grid for this geometry, denoted by $\mathcal{G}_{b c f}^{(j)}$, was shown previously in Figure 5, and consists of a background Cartesian grid in the fluid channel together with a boundary-fitted hyperbolic grid that extends around the beam. The hyperbolic grid is generated with 6 grids lines in the normal direction, and thus this grid becomes thinner as the composite grid is refined as in previous examples. The grid spacing is chosen to be approximately $h_{j}=1 /(10 j)$.

The parameters for the fluid are $\rho=1$ and $\mu=0.05$, while the parameters for the beam are $\bar{E} \bar{I}=5$, $\bar{K}=\bar{T}=\bar{K}_{1}=\bar{T}_{1}=0$, and with a range of values for the beam density $\bar{\rho}$ as noted below. The conditions on the boundaries of the fluid channel are chosen as a no-slip wall on the bottom boundary at $y=0$, a slip-wall on the top boundary at $y=y_{b}$, inflow on the left at $x=x_{a}$, and outflow on the right at $x=x_{b}$. The outflow boundary condition is a Robin condition on $p$ and extrapolation of the velocity components. The inflow velocity profile is taken to be uniform over most of the inflow boundary but parabolic near the bottom to match the no-slip boundary condition on the lower wall. The inflow profile is given as

$$
v_{1}\left(x_{b}, y, t\right)=V_{\text {in }}(y) R(t), \quad V_{\text {in }}(y)= \begin{cases}V_{\max } & \text { for } y \geq d, \\ V_{\max }(y / d)^{2} & \text { for } y<d,\end{cases}
$$

where $V_{\max }=1, d=0.2$ and $R(t)$ is the ramp function given previously in (81) so that the horizontal component of the inflow velocity transitions smoothly in time from $v_{1}=0$ at $t=0$ to $v_{1}=V_{\text {in }}(y)$ for $t>1$. The vertical component of the inflow velocity at $x=x_{a}$ is zero.
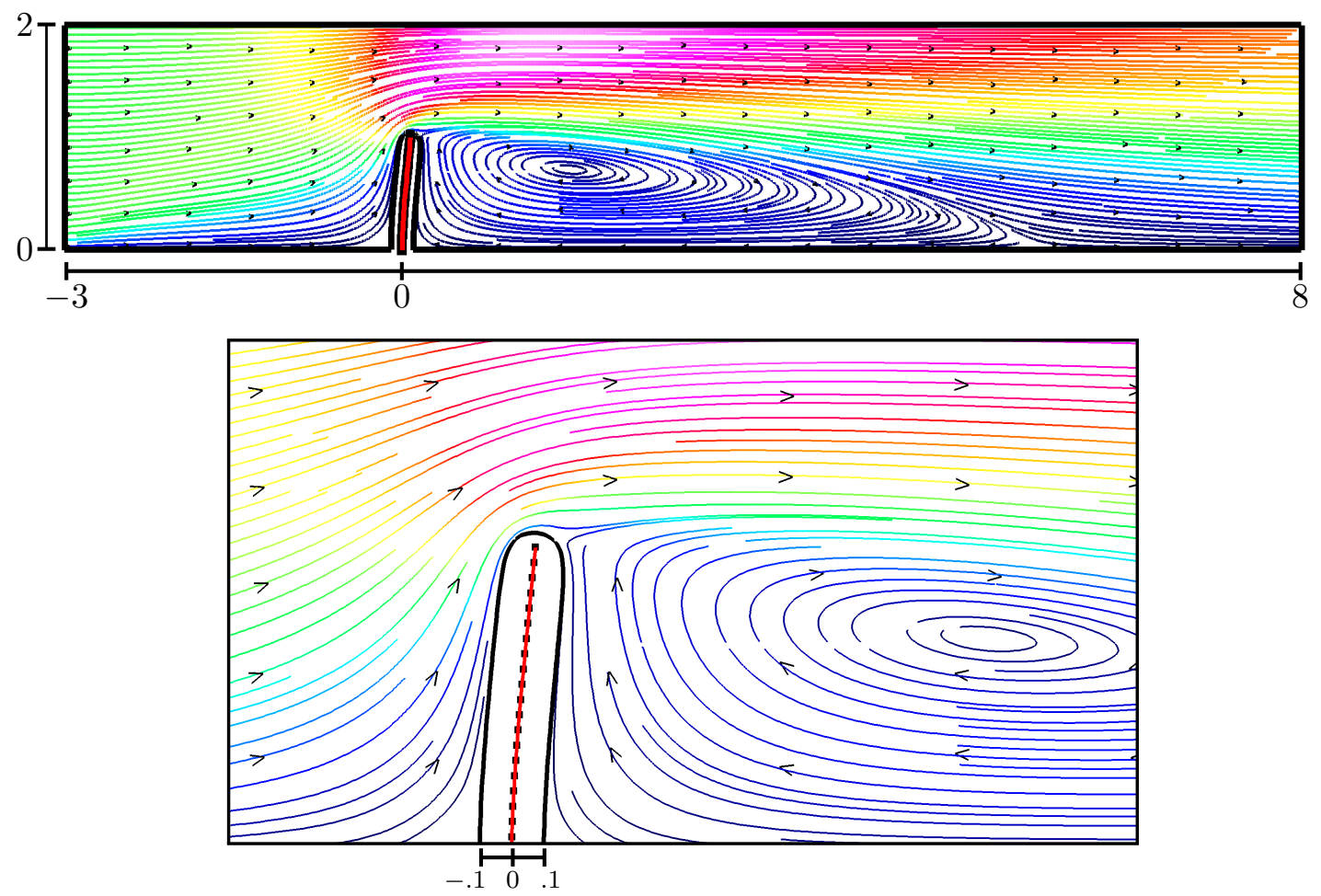

Figure 22: Light beam with $\bar{\rho}=1$ in a cross flow: Streamlines of the flow near steady state at $t=20$ computed on the composite grid $\mathcal{G}_{b c f}^{(4)}$.

Figure 23 shows the behavior of the displacement and velocity of the tip of the beam for the case of a light beam with $\bar{\rho}=1$. Solutions are computed using the AMP scheme with composite grids $\mathcal{G}_{b c f}^{(j)}, j=2,4,8$, to indicate the grid convergence. We also show the behavior of the displacement and velocity computed using the TP-SI scheme for a fine grid $\mathcal{G}_{b c f}^{(8)}$. The zoomed views show excellent grid convergence for the

scripts to run the simulations presented here will be available with the Overture software at overtureFramework.org. 
AMP scheme, and excellent agreement with the solution obtained using the TP-SI scheme. We note that the TP-SI scheme required, on average, approximately 19 sub-iterations per time-step using grid $\mathcal{G}_{b c f}^{(4)}$, and 6 sub-iterations using grid $\mathcal{G}_{b c f}^{(8)}$, with an iteration tolerance of $10^{-6}$ in both cases. Also note that substantially more sub-iterations were required when the beam was undergoing significant motion compared to when it was near steady state (see the performance results below).
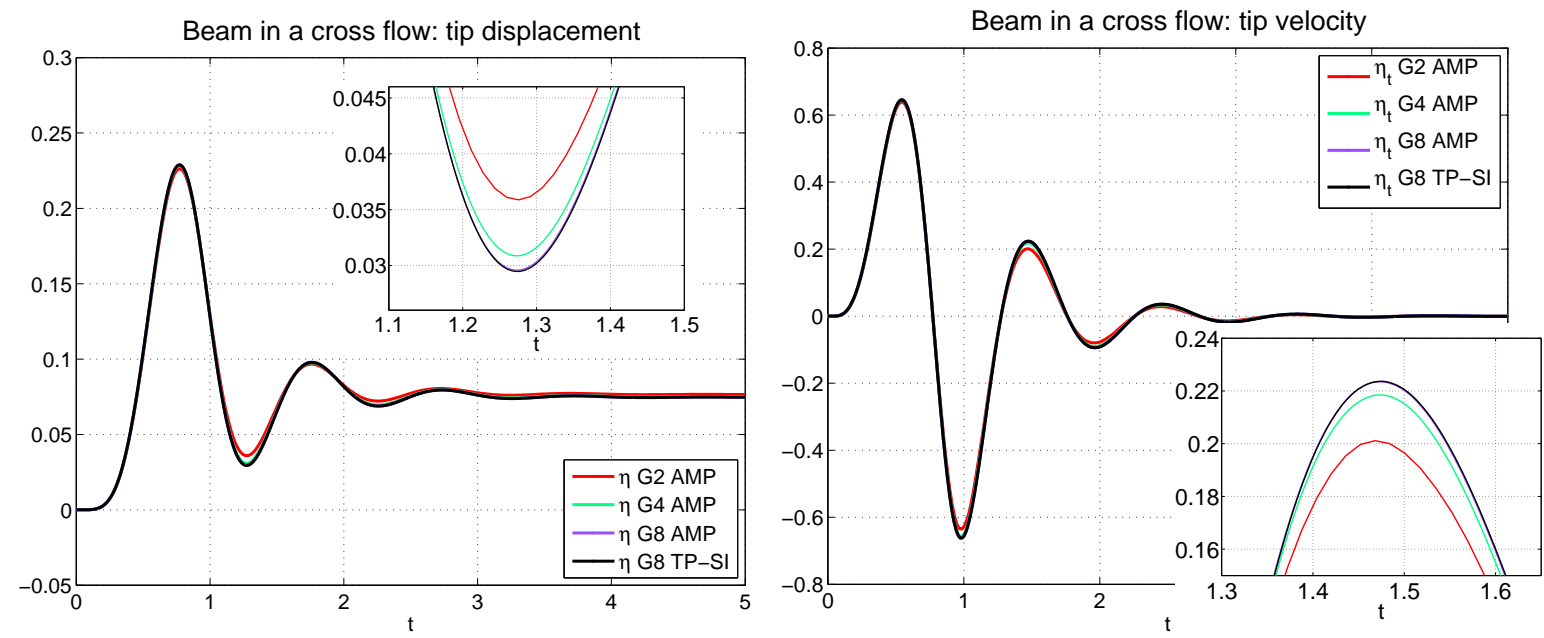

Figure 23: Light beam in a cross flow: tip displacement and velocity as the grid is refined for parameters $\bar{E} \bar{I}=5$, $\bar{\rho}=1$.

Figure 24 shows the tip displacement and velocity for beams of different densities computed using the composite grid $\mathcal{G}_{b c f}^{(4)}$. We observe damped oscillations for all beams, with the damping for the heavy beam being the weakest and its approximate period (time between successive peaks) being the largest. We also note higher-frequency sub-harmonic motion of the beam tip, especially for the heavy beam. All solutions eventually approach a steady state where the displacement of the beam tip is $\eta_{\text {tip }} \approx 0.075$ independent of the beam density.

In the absence of the fluid, the frequency, $\bar{\omega}_{0}$, and period, $\bar{\tau}_{0}$, of the lowest mode of a cantilevered beam are well known [37] and given by

$$
\bar{\omega}_{0} \approx(1.875)^{2} \sqrt{\frac{\bar{E} \bar{I}}{\bar{\rho} \bar{h} \bar{l}^{4}}}, \quad \bar{\tau}_{0}=\frac{2 \pi}{\bar{\omega}_{0}} .
$$

By comparing the computed frequency and period of the damped oscillating beam from the FSI simulation, the added-mass of the fluid can be estimated. In view of the analysis discussed in Section 6 we expect that the frequency of the beam, when accounting for added mass effects, would be approximately given by

$$
\bar{\omega}_{A M} \approx(1.875)^{2} \sqrt{\frac{\bar{E} \bar{I}}{\left(\bar{\rho} \bar{h}+M_{a}\right) \bar{l}^{4}}}, \quad \bar{\tau}_{A M}=\frac{2 \pi}{\bar{\omega}_{A M}},
$$

where $M_{a}$ is the added mass of the fluid (see equation (49)). Thus, using (84) and (85), we have the estimate

$$
M_{a}=\bar{\rho} \bar{h}\left\{\left(\bar{\tau}_{A M} / \bar{\tau}_{0}\right)^{2}-1\right\} .
$$

For $\bar{\rho}=100, \bar{h}=0.2, \bar{l}=1$ and $\bar{E} \bar{I}=5$, we find $\bar{\tau}_{0} \approx 3.57$ which is close to the period estimated from Figure 24 of $\bar{\tau}_{A M} \approx 3.6$. Using (86), we find $M_{a} /(\bar{\rho} \bar{h}) \approx .02$, which is small as expected for this heavy beam.

For a lighter beam with $\bar{\rho}=1$, we obtain $\bar{\tau}_{0} \approx 0.357$ and $\bar{\tau}_{A M} \approx .99$, which gives $M_{a} \approx 1.3$, and this value is approximately 6.5 times larger than the beam mass of $\bar{\rho} \bar{h}=0.2$. Based on the earlier mode analysis and the stability result in (65), we can estimate that the traditional scheme would become unconditionally unstable for $\bar{\rho}=M_{a} / \bar{h}=1.3 / 0.2=6.5$ for $\Delta t$ approaching zero. Computations of the full problem indicate 

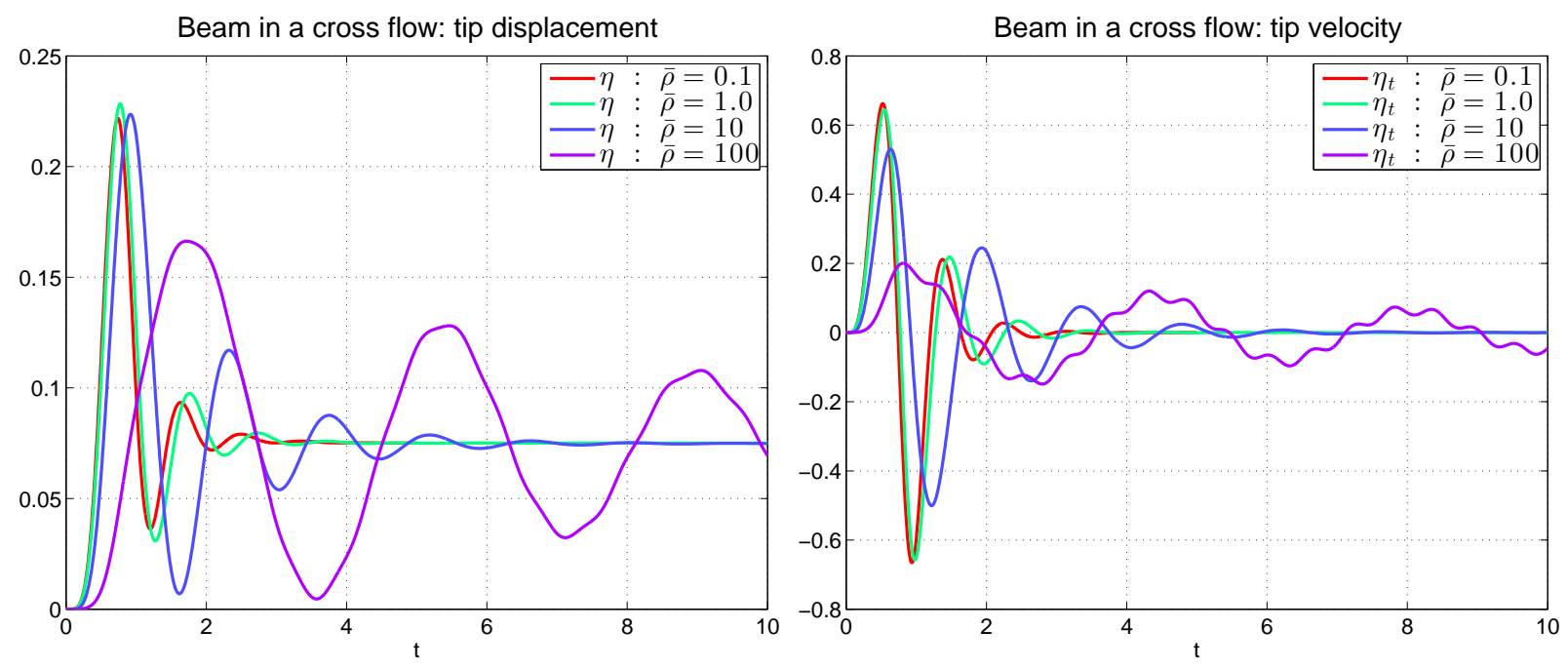

Figure 24: Beam in a cross flow: comparison of beam tip displacement and velocity for beams of different densities. The solution was computed on grid $\mathcal{G}_{b c f}^{(4)}$ with $\bar{E} \bar{I}=5$.

that the TP scheme actually becomes unstable at a larger value of $\bar{\rho} \approx 13$. This larger value is expected since the time-step for this computation is chosen based on the stability of the domain solvers, as might be done in practice, and not reduced to account for added-mass effects.

We end this section by providing a rough comparison between the run-time CPU costs for the AMP and TP-SI schemes. In this comparison the beam in a cross-flow problem is solved on the grid $\mathcal{G}_{b c f}^{(8)}(150 \mathrm{~K}$ grid points) on a single processor of a Linux desktop computer. The fluid pressure equation is solved with a bi-Conjugate-Gradient-stabilized Krylov solver and an ILU(3) preconditioner with a relative convergence tolerance of $10^{-6}$. The relative convergence tolerance for the TP-SI sub-iterations is $10^{-6}$. The table in Fig. 25 presents run-time performance results comparing the CPU times in seconds-per-step between the AMP and TP-SI schemes over a time interval when the beam is undergoing significant motion. For a relatively heavy beam, $\bar{\rho}=100$, the AMP scheme is basically the same cost, $1.33 \mathrm{~s} / \mathrm{step}$, as the TP scheme with no sub-iterations, $1.48 \mathrm{~s} /$ step. $^{9}$ This is expected since the AMP scheme adds little overhead and since the majority of CPU time is spent in solving the pressure equation. For the light beam case, $\bar{\rho}=1$, the TP-SI scheme requires an average of approximately 62 sub-iterations per time step and is approximately 8 times slower than the AMP scheme overall. The TP-SI scheme is run with an under-relaxation parameter of $\omega=.1$; this value being chosen since the scheme does not converge with $\omega=.2$ and is slower with $\omega=.15$ and $\omega=.05$. Since the solution of the pressure equation requires fewer Krylov iterations as the TP-SI sub-iterations converge (due to better initial guesses for the later sub-iterations), the TP-SI scheme turns out to be only 8 times slower than the AMP scheme even though 62 sub-iterations are used. It should also be noted that the number of sub-iterations could be reduced using an acceleration procedure such as Aitken acceleration, although the overall benefit is hard to predict given that the initial sub-iterations are most costly.

\section{Conclusions}

This article makes a significant contribution to resolving an important and long-standing difficulty for fluid-structure interaction (FSI) problems involving incompressible fluids and thin structures. Such FSI problems arise in many applications in science and engineering, and their accurate solution is an important and active field of research. In a pair of recent papers [4, 5], we have developed new added-mass partitioned

\footnotetext{
${ }^{9}$ The AMP scheme is slightly faster, perhaps due to the AMP pressure interface conditions giving a better conditioned linear system.
} 


\begin{tabular}{|c|c||c|c||c|}
\hline \multicolumn{5}{|c|}{ Run-time performance of AMP versus TP-SI } \\
\hline \hline \multirow{3}{*}{} & AMP & \multicolumn{2}{|c|}{ TP-SI } & AMP speed-up \\
\cline { 2 - 5 } & s/step & s/step & sub-its & TP-SI/AMP \\
\cline { 2 - 5 } $\bar{\rho}=1$ & 2.87 & 22.6 & 62 & 7.87 \\
\cline { 2 - 5 } $\bar{\rho}=100$ & 1.33 & 1.48 & 0 & 1.1 \\
\hline
\end{tabular}

Figure 25: Comparison of the run-time performance of the AMP scheme versus the TP-SI scheme for flow past a light and heavy beam in a channel computed using grid $\mathcal{G}_{b c f}^{(8)}(150 \mathrm{~K}$ grid points). The column labeled "s/step" gives the CPU time in seconds per time-step. The column labeled "sub-its" gives the average number of sub-iterations per time-step used in the TP-SI scheme.

(AMP) algorithms for linearized FSI problems coupling incompressible (Stokes) flows and linearly elastic bulk solids and thin structures. An important feature of the AMP algorithms is that they are stable, without subtime-step iterations, independent of added-mass effects. The present paper represents a first and important extension of the AMP algorithm in [5] to fully nonlinear FSI problems for the case of thin structures. The AMP interface conditions and time-stepping algorithm has been fully described for this class of FSI problems. The key AMP interface condition is a Robin (mixed) boundary condition for the fluid pressure, and for a general beam this becomes a generalized Robin condition that couples points on the circumference of the beam cross-section. For a two-dimensional beam with fluid on two sides, for example, this condition involves the pressure on opposite sides of the beam. While the AMP algorithm has been described for general beams, the focus has been on beams in two dimensions described by the Euler-Bernoulli (EB) equations. A normalmode stability analysis has been performed for a linearized problem involving an EB beam separating fluid domains on either side. The analysis extends that given in [5] and shows that the AMP scheme is insensitive to added-mass effects for this more general problem configuration, while a traditional partitioned scheme (without sub-time-step iterations) becomes unconditionally unstable when added-mass effects are sufficiently strong.

The general numerical framework for handling finite-amplitude deformations of the structure is based on the use of moving and deforming composite grids, previously used in the context FSI problems involving compressible flows coupled to rigid solids [30, 38], and also to linear [6] and nonlinear [7] elastic solids. The equations governing the incompressible fluid are solved using a fractional-step finite-difference scheme on deforming grids, while the equations for the EB beam are solved using either finite differences or a finiteelement approach. For the case of the finite-element beam solver, special treatment of the AMP interface conditions are required in order to couple to the finite-difference fluid solver and we have described two approaches for implementing this coupling.

An important feature of this paper is the use of benchmark problems of increasing complexity to illustrate the behavior of the new AMP algorithm. In addition to carefully verifying the stability and second-order accuracy of the present algorithm, the results of the benchmark problems can be useful to other researchers developing other FSI algorithms. The AMP scheme was first verified using manufactured solutions for the case of a deforming beam with a fluid domain on one side. The solution was next computed for a problem (with known steady state solution) of a deformed beam next to one or two pressurized fluid chambers, and for a time-dependent oscillating flat beam (with known solution). Results were obtained using a traditionalpartitioned (TP) scheme with sub-time-step iterations and these agreed with those obtained using the AMP scheme. The AMP scheme was also applied to a problem from the literature for a simplified model of an artery: solutions were computed for a traveling wave pressure-pulse propagating through a deforming channel. Grid convergence studies were performed and a comparison was made to prior results available in the literature. Lastly the motion of a cantilevered beam in a fluid channel was considered, and solutions for beams with different densities were compared and these results were used to estimate the added mass. In all benchmark cases considered the numerical solutions from the AMP algorithm remained accurate and stable even for the case of light beams when added-mass effects are large.

In future work we will consider more general nonlinear beam models as well as FSI problems involving beams, tubes, and shells in three space dimensions. 


\section{References}

[1] P. Causin, J. F. Gerbeau, F. Nobile, Added-mass effect in the design of partitioned algorithms for fluid-structure problems, Comput. Method. Appl. Mech. Engrg. 194 (2005) 4506-4527.

[2] E. H. van Brummelen, Added mass effects of compressible and incompressible flows in fluid-structure interaction, J. Appl. Mech. 76 (2) (2009) 021206-021212.

[3] Y. Yu, H. Baek, G. E. Karniadakis, Generalized fictitious methods for fluid-structure interactions: Analysis and simulations, J. Comput. Phys. 245 (2013) 317-346.

[4] J. W. Banks, W. D. Henshaw, D. W. Schwendeman, An analysis of a new stable partitioned algorithm for FSI problems. Part I: Incompressible flow and elastic solids, J. Comput. Phys. 269 (2014) 108-137.

[5] J. W. Banks, W. D. Henshaw, D. W. Schwendeman, An analysis of a new stable partitioned algorithm for FSI problems. Part II: Incompressible flow and structural shells, J. Comput. Phys. 268 (2014) 399-416.

[6] J. W. Banks, W. D. Henshaw, D. W. Schwendeman, Deforming composite grids for solving fluid structure problems, J. Comput. Phys. 231 (9) (2012) 3518-3547.

[7] J. W. Banks, W. D. Henshaw, D. W. Schwendeman, An added-mass partitioned algorithm for fluidstructure interactions of compressible fluids and nonlinear solids, (submitted to J. Comput. Phys.), Research Report LLNL-JRNL-656255, Lawrence Livermore National Laboratory (2014).

[8] M. A. Fernández, Coupling schemes for incompressible fluid-structure interaction: implicit, semiimplicit and explicit, SeMa Journal 55 (1) (2011) 59-108.

[9] J. Degroote, Partitioned simulation of fluid-structure interaction, Archives of Computational Methods in Engineering 20 (3) (2013) 185-238.

[10] M. Bukač, S. Čanič, B. Muha, A partitioned scheme for fluid-composite structure interaction problems, Journal of Computational Physics 281 (2015) 493 - 517.

[11] F. Nobile, M. Pozzoli, C. Vergara, Time accurate partitioned algorithms for the solution of fluidstructure interaction problems in haemodynamics, Computers \& Fluids 86 (0) (2013) $470-482$.

[12] J. Liu, R. K. Jaiman, P. S. Gurugubelli, A stable second-order scheme for fluid-structure interaction with strong added-mass effects, Journal of Computational Physics 270 (2014) 687 - 710.

[13] G. Guidoboni, R. Glowinski, N. Cavallini, S. Čanić, Stable loosely-coupled-type algorithm for fluidstructure interaction in blood flow, J. Comput. Phys. 228 (18) (2009) 6916-6937.

[14] S. Čanić, B. Muha, M. Bukač, Stability of the kinematically coupled $\beta$-scheme for fluid-structure interaction problems in hemodynamics, ArXiv e-print 1205.6887.

[15] M. Bukač, S. Čanič, R. Glowinski, J. Tambača, A. Quaini, Fluid-structure interaction in blood flow capturing non-zero longitudinal structure displacement, J. Comput. Phys. 235 (2013) 515-541.

[16] M. Lukáčová-Medvid'ová, G. Rusnáková, A. Hundertmark-Zaušková, Kinematic splitting algorithm for fluidstructure interaction in hemodynamics, Computer Methods in Applied Mechanics and Engineering 265 (2013) $83-106$.

[17] M. A. Fernández, Incremental displacement-correction schemes for the explicit coupling of a thin structure with an incompressible fluid, Comptes Rendus Mathematique 349 (78) (2011) 473-477.

[18] M. A. Fernández, Incremental displacement-correction schemes for incompressible fluid-structure interaction: stability and convergence analysis, Numerische Mathematik 123 (1) (2013) 21-65. 
[19] M. A. Fernández, J. Mullaert, M. Vidrascu, Explicit Robin-Neumann schemes for the coupling of incompressible fluids with thin-walled structures, Comput. Method. Appl. Mech. Engrg. 267 (2013) $566-593$.

[20] M. A. Fernández, M. Landajuela, A fully decoupled scheme for the interaction of a thin-walled structure with an incompressible fluid, Comptes Rendus Mathematique 351 (34) (2013) 161-164.

[21] M. A. Fernández, M. Landajuela, Fully decoupled time-marching schemes for incompressible fluid/thinwalled structure interaction, Rapport de recherche RR-8425, INRIA (Jan. 2014).

[22] M. A. Fernández, M. Landajuela, M. Vidrascu, Fully decoupled time-marching schemes for incompressible fluid/thin-walled structure interaction, Research Report RR-8425, Inria (Dec. 2014).

[23] W. D. Henshaw, A fourth-order accurate method for the incompressible Navier-Stokes equations on overlapping grids, J. Comput. Phys. 113 (1) (1994) 13-25.

[24] J. W. Banks, B. Sjögreen, A normal mode stability analysis of numerical interface conditions for fluid/structure interaction, Commun. Comput. Phys. 10 (2) (2011) 279-304, .

[25] W. D. Henshaw, N. A. Petersson, A split-step scheme for the incompressible Navier-Stokes equations, in: M. M. Hafez (Ed.), Numerical Simulation of Incompressible Flows, World Scientific, 2003, pp. 108-125.

[26] J. J. H. Miller, On the location of zeros of certain classes of polynomials with applications to numerical analysis, IMA J. Appl. Math. 8 (3) (1971) 397-406.

[27] J. C. Strikwerda, Finite Difference Schemes and Partial Differential Equations, Wadsworth and Brooks/Cole, 1989.

[28] W. D. Henshaw, The Overture hyperbolic grid generator, user guide, version 1.0, Research Report UCRL-MA-134240, Lawrence Livermore National Laboratory (1999).

[29] W. D. Henshaw, Ogen: An overlapping grid generator for Overture, Research Report UCRL-MA-132237, Lawrence Livermore National Laboratory (1998).

[30] W. D. Henshaw, D. W. Schwendeman, Moving overlapping grids with adaptive mesh refinement for high-speed reactive and non-reactive flow, J. Comput. Phys. 216 (2) (2006) 744-779.

[31] D. Appelö, J. W. Banks, W. D. Henshaw, D. W. Schwendeman, Numerical methods for solid mechanics on overlapping grids: Linear elasticity, J. Comput. Phys. 231 (18) (2012) 6012-6050.

[32] G. S. Chesshire, W. D. Henshaw, Composite overlapping meshes for the solution of partial differential equations, J. Comput. Phys. 90 (1) (1990) 1-64.

[33] P. J. Roache, Verification and Validation in Computational Science and Engineering, Hermosa Publishers, New Mexico, 1998.

[34] L. Formaggia, J. Gerbeau, F. Nobile, A. Quarteroni, On the coupling of 3D and 1D Navier-Stokes equations for flow problems in compliant vessels, Comput. Method. Appl. Mech. Engrg. 191 (6-7) (2001) $561-582$.

[35] W. D. Henshaw, D. W. Schwendeman, Parallel computation of three-dimensional flows using overlapping grids with adaptive mesh refinement, J. Comput. Phys. 227 (16) (2008) 7469-7502.

[36] W. D. Henshaw, Mappings for Overture, a description of the Mapping class and documentation for many useful Mappings, Research Report UCRL-MA-132239, Lawrence Livermore National Laboratory (1998).

[37] K. F. Graff, Wave Motion in Elastic Solids, Dover Publications, 1991.

[38] J. W. Banks, W. D. Henshaw, B. Sjögreen, A stable FSI algorithm for light rigid bodies in compressible flow, J. Comput. Phys. 245 (2013) 399-430. 